\title{
Tits geometry and positive curvature
}

\author{
by \\ FUQUAN FANG \\ Capital Normal University \\ Beijing, China \\ Karsten Grove \\ University of Notre Dame \\ Notre Dame, IN, U.S.A.

\section{Gudlaugur Thorbergsson} \\ Universität zu Köln \\ Köln, Germany
}

The interest in positively curved manifolds goes back to the beginning of Riemannian geometry or even to spherical and projective geometry. Likewise, the program of Tits to provide an axiomatic description of geometries whose automorphism group is a noncompact simple algebraic or Lie group goes back to projective geometry.

The presence of symmetries has played a significant role in the study of positively curved manifolds during the past two decades; see, e.g., the surveys [Gr], [Wi3] and [Z]. Not only has this resulted in a number of classification type theorems, it has also lead to new insights about structural properties, see, e.g., [VZ] and [Wi2], as well as to the discovery and construction of a new example [De], [GVZ].

Unlike [GWZ], our work here is not motivated by the quest for new examples. On the contrary, we wish to explore rigidity properties of special actions on positively curved manifolds whose linear counterparts by work of Dadok [Dad], Cartan (see [He]), Tits [Ti1] and Burns-Spatzier [BS] ultimately are described axiomatically via so-called compact spherical buildings.

The special actions we investigate are the so-called polar actions, i.e., isometric actions for which there is an (immersed) submanifold, a so-called section, that meets all orbits orthogonally. Such actions form a particularly simple, yet very rich and interesting class of manifolds and actions closely related to the transformation group itself. The

The first author is supported in part by an NSFC grant and he is grateful to the University of Notre Dame for its hospitality. The second author is supported in part by an NSF grant, a research chair at the Hausdorff Center at the University of Bonn and by a Humboldt research award. The third author is grateful to the University of Notre Dame and the Capital Normal University in Beijing for their hospitality. 
concept goes back to isotropy representations of symmetric spaces. Also, as a special case, the adjoint action of a compact Lie group on itself is polar with section a maximal torus. Its extension to general manifolds was pioneered by Szenthe in $[\mathrm{Sz}]$ and independently by Palais and Terng in [PTe], and has recently been further developed in [GZ]. Since the action by the identity component of a polar action is itself polar, we assume throughout without further comments that our group is connected. An exceptional but important special case is that of cohomogeneity one actions and manifolds, i.e., actions with 1dimensional orbit space.

The exceptional case of positively curved cohomogeneity-1 manifolds was studied in $[\mathrm{GWZ}]$ and $[\mathrm{V}]$. Aside from the rank-1 symmetric spaces, this also includes infinite families of other manifolds, most of which are not homogeneous even up to homotopy. In contrast, our main result here is the following.

THEOREM A. A polar action on a simply connected, compact, positively curved manifold of cohomogeneity at least 2 is equivariantly diffeomorphic to a polar action on a compact rank-1 symmetric space.

This is reminiscent of the situation for isoparametric submanifolds in euclidean spheres, where many isoparametric hypersurfaces are not homogeneous (see [OT], [FKM]), whereas in higher codimensions by [Th] they are the orbits of linear polar actions if they are irreducible or equivalently the orbits of isotropy representations of compact symmetric spaces by [Dad].

All polar actions on the simply connected, compact rank-1 symmetric spaces, i.e., the spheres and projective spaces, $\mathbb{S}^{n}, \mathbb{C P}^{n}, \mathbb{H}^{n}$ and $\mathbb{O P}^{2}$ were classified in [Dad] (see also $[\mathrm{EH}]),[\mathrm{PTh}]$ and $[\mathrm{GKo}]$. In all cases but $\mathbb{O P}^{2}$ they are either linear polar actions on a sphere or they descend from such actions to a projective space. By the work mentioned above by Dadok, Cartan, Tits and Burns-Spatzier, the (maximal) irreducible polar linear actions are in one-to-one correspondence with irreducible compact spherical buildings. On $\mathbb{O P}^{2}$ any polar action has either cohomogeneity 1 or 2 , and in the second case all but two have a fixed point. The latter are actions by $\mathrm{SU}(3) \mathrm{SU}(3)[\mathrm{PTh}]$ and $\mathrm{SO}(3) \mathrm{G}_{2}$ [GKo], both with a spherical triangle with angles $\left\{\frac{1}{2} \pi, \frac{1}{3} \pi, \frac{1}{4} \pi\right\}$ as orbit space. We refer to these as the exceptional (irreducible) actions on $\mathbb{O P}^{2}$.

We note that Theorem A is optimal. In fact, since the Berger-Cheeger deformation [C] preserves polarity and lower curvature bounds, there are even invariant positively curved polar metrics on any rank-1 symmetric space arbitrarily Gromov-Hausdorff close to its orbit space.

There are different steps and strategies involved in the proof of Theorem A. To guide the reader, we provide a short discussion of the key results needed in the proof. 
Our point of departure is the following description of sections and their (effective) stabilizer groups, referred to as polar groups in [GZ] and generalized Weyl groups in [Sz] and [PTe].

THEOREM B. The polar group of a simply connected, positively curved polar manifold of cohomogeneity at least 2 is a Coxeter group or a $\mathbb{Z}_{2}$ quotient thereof. Moreover, the section with this action is equivariantly diffeomorphic to a sphere, or a real projective space with a linear action, respectively.

If this linear action is irreducible, then we say that the polar $\mathrm{G}$ action is irreducible, and reducible otherwise. The above result also allows us to associate a (connected) chamber system $\mathscr{C}(M ; \mathrm{G})$ (cf. [Ti2], [R]) of type $\mathrm{M}$ (the Coxeter matrix of the associated Coxeter group), with any simply connected, positively curved polar G-manifold $M$ of cohomogeneity at least 2 . We point out that in this generality, the geometric realization of $\mathscr{C}(M ; \mathrm{G})$ is not always a simplicial complex, so not a geometry of type $\mathrm{M}$ in the sense of Tits. For this we prove the following result.

Theorem C. Let $M$ be a simply connected, positively curved polar G-manifold without fixed points, and not (equivalent to) an exceptional action on $\mathbb{O P}^{2}$. Then, the universal cover $\tilde{\mathscr{C}}(M ; \mathrm{G})$ of $\mathscr{C}(M ; \mathrm{G})$ is a spherical building.

Moreover, the Hausdorff topology on compact subsets of $M$ induces, in a natural way, a topology on $\tilde{\mathscr{C}}(M ; \mathrm{G})$ for which we prove the following result.

TheOREM D. Whenever the universal cover $\tilde{\mathscr{C}}(M ; \mathrm{G})$ of $\mathscr{C}(M ; \mathrm{G})$ is a building, it is a compact spherical building.

When the Coxeter diagram for $\mathrm{M}$ is connected, or more generally has no isolated nodes, the work of Burns and Spatzier [BS] as extended by Grundhöfer, Kramer, Van Maldeghem and Weiss [GKMW] applies, and hence $\tilde{\mathscr{C}}(M ; \mathrm{G})$ is the building of the sphere at infinity of a non-compact symmetric space $\mathrm{U} / \mathrm{K}$ of non-positive curvature, and the action of $\mathrm{K}$ on the sphere at infinity is the linear polar action whose chamber system is the building. In our case, the fundamental group $\pi$ of the cover becomes a compact normal subgroup of $\widetilde{G} \subset K$, acting freely on the sphere with quotient our manifold with the action by $\mathrm{G}=\widetilde{G} / \pi$. Moreover, the actions by $\widetilde{G}$ and $\mathrm{K}$ on the sphere are orbitequivalent. This already proves our Theorem A up to equivariant homeomorphism in this case (Theorem 4.10), and equivariant diffeomorphism follows, e.g., from the recognition theorem in [GZ]. In particular, we note that in this case $M$ is either a sphere or a quotient thereof by a Hopf action, i.e., not the Cayley plane.

In the remaining (reducible) cases (including the case of fixed points), where isolated nodes of the Coxeter diagram are present, the above mentioned extended Burns-Spatzier- 
Tits theory does not yield the desired result, and we also use more direct geometric arguments that hinge on a characterization of Hopf fibrations in our context, Lemma 6.2.

We point out that the proof of Theorem $\mathrm{C}$ above has three distinct parts, a special one of which is carried out in [FGT]. For all chamber systems of rank at least four, our constructions combined with the work of Tits gives the result (cf. Theorem 3.13). In the reducible rank three cases it follows from Theorem 7.1. In the irreducible rank-3 cases, i.e., of type $A_{3}$ and $C_{3}$, corresponding to the orbit space being a spherical triangle with angles $\left\{\frac{1}{2} \pi, \frac{1}{3} \pi, \frac{1}{3} \pi\right\}$ and $\left\{\frac{1}{2} \pi, \frac{1}{3} \pi, \frac{1}{4} \pi\right\}$, respectively, the general theory breaks down. The point of departure here (Theorem 5.1), is that in this case $\mathscr{C}(M ; \mathrm{G})$ is simplicial, thus an $A_{3}$ (resp. a $C_{3}$ ) geometry. Since all $A_{n}$ geometries are buildings by work of Tits, this completes the case of $A_{3}$. In the case of $C_{3}$, one can use an axiomatic characterization of buildings of type $\mathrm{C}_{3}$ due to Tits. This is carried out in [FGT] via reductions and the work in [GWZ]. Unlike the higher-rank case, the strategy here is to construct suitable covers and prove that they are buildings. Exactly two cases emerge (from the exceptional actions on $\mathbb{O P}^{2}$ ) where this cannot be done due to Theorem $\mathrm{D}$ and the subsequent discussion above. In fact, we conclude the following result.

TheOREM E. The chamber system $\mathscr{C}\left(\mathbb{O P}^{2}, \mathrm{G}\right)$ for an irreducible polar $\mathrm{G}$ action on $\mathbb{O P}^{2}$ is a $\mathrm{C}_{3}$ geometry whose universal cover is not a building.

The existence of $\mathrm{C}_{3}$ geometries whose universal covers are not buildings are well known in the "real estate community" (see $[\mathrm{N}])$, but the examples $\mathscr{C}\left(\mathbb{O P}^{2}, \mathrm{G}\right)$ with $\mathrm{G}=\mathrm{SU}(3) \cdot \mathrm{SU}(3)$ and $\mathrm{G}=\mathrm{SO}(3) \cdot \mathrm{G}_{2}$, which arise naturally in our context, are new.

We conclude this outline by pointing out that positive curvature is used in the general theory for two purposes: (1) To prove Theorem B (cf. §2), and (2) to establish that the associated chamber system $\mathscr{C}(M ; \mathrm{G})$ is connected, or equivalently the $\mathrm{G}$ action is primitive (cf. §3). In fact, we prove the conclusion of Theorem A for any polar action with connected chamber system and whose orbit space has positive curvature, unless it is of type $C_{3}$. In the case of $C_{3}$ (see [FGT]) we use positive curvature more extensively, in particular relying on the work in [GWZ] (alternatively, due to Theorem 5.1, this case is also covered by the classification of irreducible homogeneous geometries of finite Coxeter type $\mathrm{M}$ of rank at least 2 in $[\mathrm{KL}]$ ).

Unlike previous applications of buildings to geometry, what is essential for us is to use Tits's local approach to buildings, i.e., via chamber systems and their universal covers [Ti2], $[\mathrm{R}]$. We like to mention that this is the case also in independent simultaneous work by Lytchak [L] describing the structure of polar singular foliations of codimension at least 3 in compact symmetric spaces. In particular, in his context results similar to Theorem D and its corollary, Theorem E, were obtained. 
We point out that a corresponding theory for polar actions on non-negatively curved manifolds is significantly more involved. In particular, the concept of an "irreducible action" is not as straightforward in this case, in part since the section is no longer just a sphere or a real projective space with a reflection group (for a complete description see $[\mathrm{FG}]$ ). For example, the polar $\mathrm{T}^{2}$ action on $\mathbb{C P}^{2}$ with three fixed points induces a polar $\mathrm{T}^{2}$ action on $\mathbb{C P}^{2} \# \pm \mathbb{C P}^{2}$ with a metric of non-negative curvature and flat Klein bottle as section (see [GZ]) that should be viewed as reducible, as should actions that are not primitive.

With the appropriate notion of irreducibility we make the following conjecture.

Conjecture. An irreducible polar action on a simply connected, non-negatively curved compact manifold is equivariantly diffeomorphic to a quotient of a polar action on a symmetric space.

Another interesting direction is based on part of our work here that generalizes to curvature free settings. For example, the combinatorial content of our paper can be used in the study of general polar actions on simply connected manifolds with finite polar groups.

We have divided the paper into seven sections. Structurally, it consists of three rather different moderately intertwined parts: $\S \S 1-4$ constituting Part I, $\S 5$ (together with [FGT]) Part II, and $\S \S 6-7$ Part III. Here, Part I deals with the overall general approach and theory leading to a proof of Theorem A for all irreducible actions of cohomogeneity at least 3. Part II deals with the exceptional case of irreducibe actions of cohomogeneity 2, where the key issue for the general theory breaks down. Finally, Part III deals with all reducible cases including cohomogeneity 2. In particular, Parts I and III yield a proof of Theorem A in cohomogeneity at least 3 . In cohomogeneity 2 only the irreducible actions of type $C_{3}$ are not covered in this paper, and we refer to [FGT] (or [KL]).

The first two sections are devoted to preliminaries and an analysis of sections culminating in Theorem B, which actually provides a complete classification of positively curved manifolds with reflection groups. The chamber system associated with a polar action in positive curvature is investigated in $\S 3$. The point of departure here is that this chamber system is connected. The proof of this is based on a result about dual foliations due to Wilking [Wi2]. We conclude $\S 3$ by proving Theorem $\mathrm{C}$ in all (irreducible) cases but $\mathrm{A}_{3}$ and $\mathrm{C}_{3}$.

In $\S 4$ we equip the ingredients of Theorem $\mathrm{C}$ with a natural topology based on the classical Hausdorff topology on closed sets in a compact metric space. Our main result here is that with this topology the universal covers of our chamber systems are compact spherical buildings in the sense of Burns and Spatzier. This then in particular leads to 
a proof of Theorem $A$ for all irreducible actions but those of type $A_{3}$ and $C_{3}$.

As mentioned above, the general theory for compact spherical buildings breaks down for reducible actions in general (the ones for whom the Coxeter diagram has isolated nodes). The proof of Theorem $\mathrm{A}$ for such actions is carried out in $\S 6$ and $\S 7$. As a key input, we provide in $\S 6$ a characterization of Hopf fibrations in our context which is of independent interest. This immediately yields Theorem A for the special case where fixed points are present. The reducible case where no fixed points are present is dealt with in $\S 7$.

For basic facts and tools involving critical point theory for non-smooth distance functions and convex sets in positive curvature that will be used freely we refer to $[\mathrm{Pe}$, Chapter 11].

It is our pleasure to thank Linus Kramer and Alexander Lytchak for constructive discussions and comments. Likewise, we are grateful to an anonymous referee for constructive comments and a suggestion that lead to a significant simplification of the proof of the Hopf lemma (Lemma 6.2), and subsequent ramifications.

\section{Preliminaries}

We will begin by giving a brief description of known facts for general polar manifolds (cf. e.g. [GZ] and [HPTT] for further information). We observe that under fairly mild restrictions, there is a general so-called chamber system naturally associated with such actions. We will end the section with a description of such systems, and the special case of Coxeter systems.

Throughout the paper, $\mathrm{G}$ will be a compact connected Lie group acting isometrically on a connected compact Riemannian manifold $M$ in a polar fashion. By definition, there is a section $\Sigma$, i.e., an immersion $\sigma: \Sigma \rightarrow M$ of a connected manifold $\Sigma$, whose image intersects all G-orbits orthogonally. Moreover, we demand that $\sigma$ is a section without a subcover section, i.e. $\sigma$ does not factor through a covering $\Sigma \rightarrow \Sigma^{\prime} \rightarrow M$. Obviously g $\sigma$ is a section for any $\mathrm{g} \in \mathrm{G}$, and $\mathrm{G} \sigma(\Sigma)=M$. Clearly, $\Sigma$ has the same dimension as the orbit space $M^{*}:=M / \mathrm{G}$, i.e., the cohomogeneity of the action, or the codimension of principal orbits, $\mathrm{G} / \mathrm{H} \subset M$. If not otherwise stated, it is understood that $0<\operatorname{dim} M^{*}<\operatorname{dim} M$. This eliminates general actions by discrete groups, and general transitive actions. In addition, we also assume that $M$ is not a product where $\mathrm{G}$ acts trivially on one of the factors. In general, we will denote the image of a subset $X \subset M$ under the orbit map by $X^{*} \subset M^{*}$.

The following facts are simple and well known (cf. [Sz] and [PTe]):

- Any section is totally geodesic.

- The slice representation of any isotropy group $\mathrm{K} \subset \mathrm{G}$ is a polar representation. 
Recall here that if $\mathrm{K}$ fixes $p \in M$, then the slice representation of $\mathrm{K}$ is the action of $\mathrm{K}$ by differentials on the normal space in $T_{p} M$ to the tangent space $T_{p}(\mathrm{G} p)$ of its orbit $\mathrm{G} p$. We often restrict this further to the subspace $T_{p}^{\perp}$ perpendicular also to the fixed-point set $T_{p} M^{\mathrm{K}}$. This is also a polar representation.

Fix a section $\sigma: \Sigma \rightarrow M$ and a point $p \in \Sigma$ corresponding to a principal $\mathrm{G}$ orbit, i.e., $\mathrm{G} \sigma(p)$ is a principal orbit with isotropy group $\mathrm{H}=\mathrm{G}_{\sigma(p)}$. The stabilizer subgroup $\mathrm{G}_{\sigma(\Sigma)} \subset \mathrm{G}$ of $\sigma(\Sigma)$ induces an action on $\Sigma$. Clearly, $\mathbf{H}$ is the kernel of that action, and we refer to $\Pi:=\mathrm{G}_{\sigma(\Sigma)} / \mathrm{H}$ as the polar group associated with the section $\sigma$. Recall the following facts:

- For any $q \in \Sigma, \sigma_{*}\left(T_{q} \Sigma\right) \subset T_{\sigma(q)} M$ is a section of the polar representation, the slice representation of $\mathrm{G}_{\sigma(q)}$, and the associated polar group is the isotropy group $\Pi_{q}$.

- $\Pi$ is a discrete subgroup of $\mathrm{N}(\mathrm{H}) / \mathrm{H}$ acting properly discontinuously on $\Sigma$ with trivial principal isotropy group.

- $M^{*}=\Sigma^{*}:=\Sigma / \Pi$ is an orbifold.

In complete generality, the structure of $M$ and its $\mathrm{G}$ action is encoded in the section $\Sigma$, the polar group $\Pi$ and its actions on $\Sigma$ and $\mathrm{G} / \mathrm{H}$, and the G-isotropy groups along $\Sigma$. Although in general, $\Pi$ can be any discrete subgroup of a Lie group, typically singular orbits are present, in which case there is a non-trivial normal subgroup $W \subset \Pi$ generated by reflections $r_{i}$ associated with maximal singular isotropy groups $\mathrm{K}_{i} \subset \mathrm{G}$ along $\Sigma$ (cf. $[\mathrm{GZ}]$ ). We refer to any group generated by reflections as a reflection group. We stress that here $r: \Sigma \rightarrow \Sigma$ is called a reflection if $r$ has order 2, and at least one component of the fixed-point set has codimension 1. The codimension-1 components $\Lambda_{\mathrm{r}} \subset \Sigma$ of the fixed-point set $\Sigma^{r}$ are referred to as the mirrors of $r$. A connected component $c$ of the complement of all mirrors is called an (open) chamber of $\Sigma$. We denote the closure of an open chamber by $C=\bar{c}$ and refer to it simply as a chamber. Again, we stress that this kind of terminology is usually reserved to the situation where the complement of a mirror has two connected components interchanged by the reflection. Note that the latter is automatic for reflections on a simply connected manifold.

It is clear that $W$ acts transitively on the set of open chambers of $\Sigma$, but the stabilizer group $\mathrm{W}_{c}$, which we will call the chamber group, may be non-trivial when the section is not simply connected (cf. Example 1.1 and Theorem 1.2). Clearly, $\Sigma / \mathrm{W}=C / \mathrm{W}_{c}$. Moreover, the boundary $\partial C=C \backslash c$ of a chamber $C$ is the union of its chamber faces, where a chamber face is an intersection $C \cap \Lambda$ with a mirror $\Lambda$ such that $C \cap \Lambda$ is the closure of a non-empty open subset of $\Lambda$. More generally, a face of a chamber $C$ is an intersection $C \cap \Lambda$ with a mirror $\Lambda$. Faces that are not chamber faces play no role in this paper. We will therefore frequently simply write "faces" when we mean "chamber faces".

The following examples illustrate these concepts and are relevant for our subsequent discussion about positive curvature. 
Example 1.1. Consider the following groups $\mathrm{W}$ acting on $\mathbb{S}^{2}$ as well as on $\mathbb{R} \mathbb{P}^{2}$.

(1) $W=A_{1}=\langle r\rangle$, where $r$ is the reflection in the equator.

On $\mathbb{S}^{2}$ there is one mirror and two open chambers, the open upper and lower hemispheres interchanged by $r$. Their closure is the orbit space $\mathbb{S}^{2} / W$. There is one face, its boundary circle (and it coincides with the mirror of $r$ ).

On $\mathbb{R} \mathbb{P}^{2}$ there is one mirror and one open chamber and it is preserved by $r$. Its closure is all of $\mathbb{R} \mathbb{P}^{2}$ and the orbit space $\mathbb{R} \mathbb{P}^{2} / \mathrm{W}$ is the cone on its boundary circle, the cone point corresponds to the isolated fixed point of $r$ on $\mathbb{R} \mathbb{P}^{2}$ (in the chamber). There is one face, the whole boundary (and it coincides with the mirror of $r$ ).

Note that the action of $\mathrm{W}$ on $\mathbb{R} \mathbb{P}^{2}$ lifts to the action of $\mathrm{W}$ on $\mathbb{S}^{2}$. If we extend this action by $-\mathrm{id}$, the extended group action induces the same action on the base, and now has the same orbit space.

(2) $W=A_{1} \times A_{1}=\left\langle r_{0}, r_{2}\right\rangle$ where $r_{0}$ and $r_{2}$ are reflection in two great circles making an angle $\frac{1}{2} \pi$.

On $\mathbb{S}^{2}$ there are two mirrors and four open chambers. Their closure is the orbit space $\mathbb{S}^{2} / \mathrm{W}$, a spherical right-angled biangle. There are two faces, each of which is also a chamber face. Their intersection is the intersection of mirrors and coincides with the fixed-point set Fix $(\mathrm{W})$.

On $\mathbb{R} \mathbb{P}^{2}$ there are three mirrors and four open chambers. In fact, the "rotation" $r_{0} r_{2}$ on $\mathbb{S}^{2}$ induces a reflection on $\mathbb{R} \mathbb{P}^{2}$. The closure of an open chamber is the orbit space $\mathbb{R} \mathbb{P}^{2} / \mathrm{W}$, a right-angled spherical triangle. There are three faces, each of which is also a chamber face. The intersection of all mirrors is empty, but each vertex of the orbit space triangle correspond in this case to a fixed point of $\mathrm{W}$.

In this case, the lifted action of $W$ on $\mathbb{R} \mathbb{P}^{2}$ to $\mathbb{S}^{2}$ contains a rotation of angle $\pi$. Again, the extended action by -id defines the same action on $\mathbb{R P}^{2}$, but on $\mathbb{S}^{2}$ the action has three reflections, and of course the same orbit space. In other words, the reflection group on $\mathbb{S}^{2}$ generated by the lift of all the reflections in $\mathbb{R} \mathbb{P}^{2}$ contains the antipodal map in this case as opposite to the first case.

(3) $\mathrm{W}=\mathrm{A}_{2}=\left\langle\mathrm{r}_{0}, \mathrm{r}_{3}\right\rangle$ where $\mathrm{r}_{0}$ and $\mathrm{r}_{3}$ are reflection in two great circles making an angle $\frac{1}{3} \pi$.

On $\mathbb{S}^{2}$ there are three mirrors and six open chambers. Their closure is the orbit space $\mathbb{S}^{2} / \mathrm{W}$, a spherical biangle with angle $\frac{1}{3} \pi$. There are two faces, each of which is also a chamber face. Their intersection is the intersection of mirrors and coincides with the fixed-point set Fix $(W)$. 
On $\mathbb{R P}^{2}$ there are three mirrors and three open chambers. The closure of an open chamber is a spherical biangle with angle $\frac{1}{3} \pi$, where the two vertices have been identified! The stabilizer $\mathrm{W}_{c}$ of a chamber has order 2 , fixes the "mid point" of $C$ and rotates $C$ to itself, mapping one chamber face to the other. The orbit space has one face with one singular point, the fixed point of $\mathrm{W}$, and one interior singular point, the fixed point of $\mathrm{W}_{c}$.

In this case, the reflection group obtained by lifting the reflections in $\mathbb{R P}^{2}$ to reflections in $\mathbb{S}^{2}$ does not contain the antipodal map. If we extend it by the antipodal map we get the same action on $\mathbb{R P}^{2}$ and the orbit spaces are, of course, the same as well.

(4) Consider a linear cohomogeneity-1 action on a sphere with orbit space of length $\pi / i, i=2,3$. The suspended action and its induced action on the real projective space have sections and polar groups as presented in (2) and (3) above.

Note that if $\Pi=\mathrm{W}$ and the chamber group $\mathrm{W}_{c}$ is trivial, it follows that $C$ is isometrically identified with $\Sigma / \mathrm{W}=M / \mathrm{G}$, and that $\mathrm{W}$ acts simply transitively on the set of closed chambers of a fixed section $\Sigma$. Moreover, $G$ acts transitively on the set of all chambers in all sections of $M$, i.e.

$$
M=\bigcup_{\mathrm{g} \in \mathrm{G}} g C,
$$

and this set of chambers is $\mathrm{G} / \mathrm{H}$ as a set. The chamber faces $F_{i}, i=1, \ldots k$, of $C$ correspond to a set of generators $r_{i}$ for $\mathrm{W}$. This way all faces of chambers $g C, g \in \mathrm{G}$, of $M$ get labeled consistently, so that $\mathrm{G}$ is label-preserving. Now define two chambers $g_{1} C$ and $g_{2} C$ to be $i$-adjacent if they have a common $i$ face $g_{1} F_{i}=g_{2} F_{i}$. This relation among the set of chambers in $M$, (resp. all chambers in a fixed section $\Sigma$ ) make both of these sets into a chamber system $\mathscr{C}(M, \mathrm{G})$ (resp. $\mathscr{C}(\Sigma, \mathrm{W})$ ) according to the following definition (see, e.g., [Ti2] and $[\mathrm{R}])$.

An (abstract) chamber system over $I=\{1, \ldots, k\}$ is a set $\mathscr{C}$ together with an $i$ partition of $\mathscr{C}$ for every $i \in I$. Elements $C, C^{\prime} \in \mathscr{C}$ in the same part of the $i$-partition, are said to be $i$-adjacent which is written as $C \sim_{i} C^{\prime}$. The elements of $\mathscr{C}$ are called chambers.

We will use the following standard terminology in the next sections.

A gallery in $\mathscr{C}$ is a sequence $\Gamma=\left(C_{0}, \ldots, C_{m}\right)$ in $\mathscr{C}$ such that $C_{j}$ is $i_{j}$-adjacent to $C_{j+1}$ for every $0 \leqslant j \leqslant m-1$. Here the word $f=i_{0} i_{2} \ldots i_{m-1}$ in $I$ is referred to as the type of the gallery. If we want to indicate this type, we write $\Gamma_{f}$ rather than just $\Gamma$. If the $i_{j}$ 's belong to a subset $J$ of $I$, we call $\Gamma=\left(C_{0}, \ldots, C_{m}\right)$ a $J$-gallery. A subset $\mathcal{B}$ of a chamber system $\mathscr{C}$ is said to be connected (or J-connected) if any two chambers in it can be connected 
by a gallery (or a $J$-gallery). The $J$-connected components of $\mathscr{C}$ are called $J$-residues. The rank of a $J$-residue is the cardinality of $J$ and its corank is the cardinality of $I \backslash J$. Given residues $R$ and $S$ of types $J$ and $K$ respectively, we say that $S$ is a face of $R$, if $R \subset S$ and $J \subset K$.

Note that for chamber systems $\mathscr{C}(M, \mathrm{G})$ as above, if two mirrors $\Lambda_{i}$ and $\Lambda_{j}$ in $\Sigma$ corresponding to two reflections $r_{i}$ and $r_{j}$ on $\Sigma$ intersect, then $\left(r_{i} r_{j}\right)^{m_{i j}}=1$ for some finite integer $m_{i j}>1$. In fact, $r_{i}, r_{j} \in W_{p}$ the reflection group of the polar representation of the isotropy group $\mathrm{G}_{p}$ for $p \in \Lambda_{i} \cap \Lambda_{j}=: \Lambda_{i j}$, so $\left\langle\mathrm{r}_{i}, \mathrm{r}_{j}\right\rangle$ is a dihedral group, and the angle between $\Lambda_{i}$ and $\Lambda_{j}$ is $\pi / m_{i j}$. In fact, in our case $m_{i j}$ is limited to 2,3 , 4, or 6 , since these are the possibilities for isotropy representations of symmetric spaces, and moreover no exceptional orbits are present (Theorem 2.7).

Recall that a symmetric $k \times k$ matrix $\mathrm{M}=\left(m_{i j}\right)$ with entries from $\mathbb{N} \cup\{\infty\}$, with $m_{i i}=1$ for all $i \in I$, and $m_{i j}>1$ if $i \neq j$ is called a Coxeter matrix.

Pictorially, $\mathrm{M}$ is given by its so-called diagram, which consists of one node for each $i \in I$ and $m_{i j}-2$ lines joining the $i$ and $j$ nodes.

The associated Coxeter group of type $\mathrm{M}$ is the group $\mathrm{W}(\mathrm{M})$ given by generators and relations as

$$
\left.\mathrm{W}(\mathrm{M})=\left\langle\left\{r_{1}, \ldots, r_{k}\right\}\right|\left(r_{i} r_{j}\right)^{m_{i j}}=1 \text { for all } i, j \in I \text { such that } m_{i j} \text { is finite }\right\rangle
$$

The pair $(\mathrm{W}(\mathrm{M}), S)$, where $S=\left\{r_{1}, \ldots, r_{k}\right\}$, is called the Coxeter system of type $\mathrm{M}$, and $k$ is referred to as its rank. The elements of $\mathrm{W}(\mathrm{M})$ that are conjugate to elements in $S$ are called reflections.

Associated with a Coxeter system $(\mathrm{W}(\mathrm{M}), S)$, where $S=\left\{r_{1}, \ldots, r_{k}\right\}$ and $I=\{1, \ldots, k\}$, there is a natural chamber system $\mathscr{C}(\mathrm{W})$ : One defines $i$-adjacency for $i \in I$ to be $w \sim_{i} w r_{i}$, i.e., each part in the $i$-partition of $\mathrm{W}$ consists of two elements. Notice that $\mathrm{W}$ is connected since $S$ generates W. There is a partial order among residues defined by setting $S \leqslant R$ if $S \supset R$. The residues $T$ for which $S \leqslant T$ implies $S=T$ are called the vertices of $\mathscr{C}(\mathrm{W})$. Denote the set of vertices by $\mathcal{V}$. With a residue $S$, one associates the subset $S^{\prime} \subset \mathcal{V}$ defined by $S^{\prime}=\{v \in \mathcal{V} \mid v \leqslant S\}$, and call $S^{\prime}$ an $i$-simplex if its cardinality is $i+1$. The set simplices in $\mathcal{V}$ is denoted by $\Delta(\mathrm{W})$.

The Coxeter complex $\Delta(\mathrm{W})$ associated with a Coxeter system $(\mathrm{W}(\mathrm{M}), S)$ also provides an example of an (abstract) simplicial complex:

Recall that an (abstract) simplicial complex is a non-empty family $\mathcal{S}$ of finite subsets (called simplices) of a set $V$ such that $\{v\} \in \mathcal{S}$ for every $v \in V$ and every subset of a simplex in $\mathcal{S}$ is a simplex in $\mathcal{S}$ (called a face). (The simplices consisting of one element are called vertices.) 
One has the following general facts (see e.g. [Dav, p.179, Theorem 10.1.5 and Lemma 10.1.6]).

THEOREM 1.2. For any reflection group $\mathrm{W}$ acting on a simply connected Riemannian manifold $\Sigma,(\mathrm{W}, S)$ is a Coxeter system, where $S$ are reflections in $\mathrm{W}$ corresponding to the faces of a chamber $C$, and $\mathrm{W}_{c}$ is trivial. If $\Sigma$ is compact, $\mathrm{W}$ is finite and isomorphic to a spherical reflection group.

Remark 1.3. The Coxeter groups that we will deal with in this paper will all be finite. By a theorem of Coxeter, Coxeter systems of rank $k+1$ are in one-to-one correspondence with finite subgroups of $\mathrm{O}(k+1)$ that are generated by reflections in hyperplanes of $\mathbb{R}^{k+1}$ and only fix the origin. Such groups have been classified. Let $(\mathrm{W}, S)$ be a Coxeter system of rank $k$ acting as a reflection group on $\mathbb{R}^{k+1}$, and consider its restriction to $\mathbb{S}^{k}$. In this case mirrors are of course great spheres $\mathbb{S}^{k-1}$, and the Coxeter group $\mathrm{W}$ acts simply transitively on the set of chambers. Each chamber is a spherical $k$-simplex and the corresponding triangulation of $\mathbb{S}^{k}$ is the geometric realization of the Coxeter complex $\Delta(\mathrm{W})$ associated with a Coxeter system $(\mathrm{W}, S)$.

The geometry of this representation is also reflected in the Coxeter diagram of $\mathrm{M}$. For example, this diagram is connected if and only if this action is irreducible. Each node corresponds to a codimension- 1 face simplex, $i$, and $\pi / m_{i j}$ is the angle between the corresponding $i$ and $j$ faces of the $k$-simplex $\mathbb{S}^{k} / \mathrm{W}$. The Coxeter diagram for the isotropy group of $\mathrm{W}$ at the vertex opposite to face $i$ is obtained from the Coxeter diagram of $\mathrm{W}$ by removing the $i$ th node.

We note that the chambers for the Coxeter system $(\mathrm{W}, S)$ in Theorem 1.2 when $\Sigma$ is a compact $k$ manifold combinatorially are the same as the spherical $k$ simplices of its representation above. Geometrically, it follows in particular that all angles in a chamber of $\Sigma$ are the same as the corresponding angles in the spherical simplex.

Although $A_{2}$ is an irreducible Coxeter group, we point out that all the linear 3dimensional representations presented in Example 1.1 above are reducible. We conclude this section with important examples of irreducible Coxeter groups.

Example 1.4. Finite Coxeter groups that are isomorphic to finite and irreducible reflection groups acting on $\mathbb{R}^{3}$ will play a special role in some of our proofs. There are three such groups that in the classification of finite Coxeter (or reflection) groups are given the symbols $A_{3}, C_{3}$, and $H_{3}$.

The group $A_{3}$ is isomorphic to the symmetric group on four letters. It is the group of symmetries of a regular tetrahedron. Its order is 24 . The 2 -simplexes in the triangulation explained above have angles $\frac{1}{2} \pi, \frac{1}{3} \pi$ and $\frac{1}{3} \pi$ at the vertices. 
The group $C_{3}$ is the symmetry group of a regular cube (or dually of a regular octahedron). Its order is 48 . The 2 -simplices in the triangulation have angles $\frac{1}{2} \pi, \frac{1}{3} \pi$ and $\frac{1}{4} \pi$ at the vertices.

The group $\mathrm{H}_{3}$ is the symmetry group of a regular dodecahedron (or dually of a regular icosahedron). Its order is 120 . The 2-simplices in the triangulation have angles $\frac{1}{2} \pi, \frac{1}{3} \pi$ and $\frac{1}{5} \pi$ at the vertices. Note that the occurrence of the angle $\frac{1}{5} \pi$ excludes $\mathrm{H}_{3}$ as a Coxeter group of a polar action.

\section{Sections and Coxeter groups}

We assume from now on that $M$ is a positively curved polar G-manifold of cohomogeneity at least 2 . This will yield strong restrictions on all of the basic items presented in $\S 1$. In particular, we will prove that sections are either spheres or real projective spaces.

When $M$ is simply connected, we show that the polar group is a Coxeter group when the section is a sphere, and a $\mathbb{Z}_{2}$ quotient of such a group when the section is a real projective space; in either case the action is linear, as stated in Theorem B of the introduction.

The starting point is the following.

Lemma 2.1. (Singular Orbit) Any positively curved polar G-manifold has singular orbits.

Proof. If all orbits have maximal dimension, the normal distribution is globally defined and integrable with leaves the sections of $M$. Since, in particular, the sectional curvature of $M$ is non-negative, it now follows from [Wa, Theorem 1.3] that the orbits of $\mathrm{G}$ are totally geodesic, and that the metric on $M$ locally is a product metric. This is a contradiction since the sectional curvature of $M$ is actually positive.

Remark 2.2. For a non-negatively curved polar G-manifold, the same conclusion holds unless $M=\Sigma \times_{\Pi} \mathrm{G} / \mathrm{H}$ is locally metrically a product. If in addition $M$ is simply connected, then $M=\Sigma \times \mathrm{G} / \mathrm{H}$ with a product metric.

From $\S 1$, we know in particular that the reflection group $W \subset \Pi$ is non-trivial and that $\partial M^{*}=\partial \Sigma^{*}$ is non-empty. This is already sufficient to prove the following result.

Proposition 2.3. (Section) Let $M$ be a compact, positively curved polar manifold. Then, any section $\Sigma$ is diffeomorphic to either a sphere $\mathbb{S}^{k}$ or a real projective space $\mathbb{R} \mathbb{P}^{k}$. In particular, the polar group $\Pi$ is finite.

Proof. Let $\mathrm{r}$ be a reflection, with mirror $\Lambda$ and a component $E \subset \Lambda$. Since the curvature is positive, the (local) distance function to $E$ is strictly concave. In particular, 
the complement $\Sigma \backslash D_{\varepsilon}(E)$ of a small tubular neighborhood of $E$ is a (locally) convex set with boundary $\partial D_{\varepsilon}(E)$. This set either has one or two components corresponding to the boundary having one or two components. In either case, each component is a disc by the standard "soul argument", and in fact $E=\Lambda$. The key fact here is that the distance function to the boundary is strictly concave, and hence has a unique point at maximal distance called the soul point. Moreover, the distance function to the soul point has no critical points. For the arguments and constructions below it is also important that the distance function is $r$ invariant.

In the case where $\Sigma \backslash D_{\varepsilon}(E)$ has two components, $\Lambda=E$ separates $\Sigma$ into two manifolds $V_{+}$and $V_{-}$, each with $\Lambda$ as a totally geodesic boundary. In this case, the isometry $r$ interchanges $V_{+}$and $V_{-}$. Moreover, a diffeomorphism $\phi$ from the upper hemisphere $\mathbb{D}_{+}^{k}$ of $\mathbb{S}^{k}$ to $V_{+}$can be chosen so that the north pole of $\mathbb{D}_{+}^{k}$ goes to the soul point of $V_{+}$, and the image of the gradient lines to the north pole of $\mathbb{D}_{+}^{k}$ are "radial" near $E$ and the soul point. The map $\Phi: \mathbb{S}^{k} \rightarrow \Sigma$ defined by $\Phi=\phi$ on $\mathbb{D}_{+}^{k}$ and $\Phi=\mathrm{r} \phi \varrho$ on $\mathbb{D}_{-}^{k}$ is a diffeomorphism which is equivariant relative to the reflections $\varrho$ in the equator of $\mathbb{S}^{k}$ and $r$ on $\Sigma$.

In the case where $\Sigma \backslash D_{\varepsilon}(E)$ has one component, $r$ fixes its soul point and acts freely elsewhere. In fact, $r$ clearly acts freely in $D_{\varepsilon}(E) \backslash E$, so by convexity, $r$ can only have isolated fixed points in $\Sigma \backslash D_{\varepsilon}(E)$. Moreover, if there was an isolated fixed point in addition to the soul point, a minimal geodesic between them would be reflected to a closed geodesic which is impossible by convexity. In particular, $\Lambda=E=\mathbb{R} \mathbb{P}^{k-1}$ and $\Sigma$ has fundamental group $\mathbb{Z}_{2}$. In the 2-fold universal cover $\widetilde{\Sigma}$ of $\Sigma$, the lift $\tilde{\Lambda}$ splits $\widetilde{\Sigma}$ into two convex components $V_{+}$and $V_{-}$with common totally geodesic boundary $\tilde{\Lambda}$, as in the first part. The reflection $r$ lifts to a reflection $\tilde{r}$ interchanging $V_{+}$and $V_{-}$, each being mapped isometrically by the projection map to $\Sigma \backslash \Lambda$ (see also Remark 2.4). Choosing a diffeomorphism $\phi$ say from the upper hemisphere $\mathbb{D}_{+}^{k}$ of $\mathbb{S}^{k}$ to $V_{+}$as before, the map $\tilde{\Phi}: \mathbb{S}^{k} \rightarrow \widetilde{\Sigma}$ defined by $\tilde{\Phi}=\phi$ on $\mathbb{D}_{+}^{k}$ and $\tilde{\Phi}=\mathbf{r} \phi \tilde{\varrho}$ on $\mathbb{D}_{-}^{k}$ is a diffeomorphism which is equivariant relative to the reflection $\tilde{\varrho}$ on $\mathbb{S}^{k}$ and $\tilde{r}$ on $\tilde{\Sigma}$. In addition, by construction, equivariant relative to the antipodal map -id on $\mathbb{S}^{k}$ and the deck transformation a of $\widetilde{\Sigma}$. We conclude that $\tilde{\Phi}$ induces a diffeomorphism $\Phi: \mathbb{R P}^{k} \rightarrow \Sigma$ which is equivariant relative to the reflections $\varrho$ on $\mathbb{R P}^{k}$ induced from $\varrho$ and $r$ on $\Sigma$.

Remark 2.4. During the proof of the result above we note in particular that if $r \in \mathrm{W}$ is a reflection of the section $\Sigma$ with mirror $\Lambda$, then:

- If $\Sigma$ is a sphere, $\operatorname{Fix}(r)=\Lambda$ and $\Lambda$ is a codimension- 1 sphere.

- If $\Sigma$ is a projective space, $\operatorname{Fix}(r)=\Lambda \cup\{s\}$, where $s$ is the soul point at maximal distance to $\Lambda$, and $\Lambda$ is a real projective space of codimension 1. Note in addition that $r$ also lifts to a map-preserving $V_{ \pm} \subset \widetilde{M}$ and acting as a on $\tilde{\Lambda}$

In particular, mirrors are connected, and if $\Pi=\langle r\rangle$, the result above gives a complete 
equivariant description of $(\Sigma, \Pi)$.

The proof above also allows us to derive further information about the reflection group $\mathrm{W}$ and the corresponding open chambers and orbit space $\Sigma / \mathrm{W}$.

Lemma 2.5. (Sphere chamber) Let $\Sigma$ be a $k$-dimensional sphere and $c$ be an open chamber. Then, the following statements hold.

- Intersections of mirrors are spheres, and the closure $C$ of $c$ is a convex set in $\Sigma$.

- There are at most $k+1$ chamber faces, and the intersection of all of them is $\operatorname{Fix}(\mathrm{W})$.

- If there are $k+1$ chamber faces, then $C$ is a $k$-simplex, and $\operatorname{Fix}(\mathrm{W})=\varnothing$.

- If there are $\ell+1<k+1$ chamber faces, then $C$ is the join of $\operatorname{Fix}(\mathrm{W})$ with an Ł-simplex.

Moreover, $C$ is a fundamental domain for $\mathrm{W}$ and $\Sigma / \mathrm{W}=C$.

Proof. If there is only one mirror $\Lambda$ corresponding to one reflection $r$, then we have $\mathrm{W}=\langle\mathrm{r}\rangle=\mathbb{Z}_{2}$ and $C$ is a closed convex disc with boundary $\Lambda=\mathrm{Fix}(\mathrm{W})$. In particular, $C$ is a join of $\operatorname{Fix}(\mathrm{W})$ with a 0 -simplex, the soul point $s$ of $C$.

Now consider any two reflections, $\mathrm{r}_{i}, i=1,2$, with corresponding mirrors $\Lambda_{i}$. If $p$ belongs to $\Lambda_{12}:=\Lambda_{1} \cap \Lambda_{2}=\operatorname{Fix}\left(\left\langle r_{1}, r_{2}\right\rangle\right)$, clearly $r_{i}$ belongs to the reflection group $W_{p}$ of the polar representation of the isotropy group $G_{p}$. In particular, $\left\langle r_{1}, r_{2}\right\rangle$ is a dihedral group, and the angle between $\Lambda_{1}$ and $\Lambda_{2}$ is $\pi / k$ for some integer $k$. In particular, the intersection $\Lambda_{12}$ is a codimension-1 totally geodesic submanifold of either mirror $\Lambda_{i}$, and hence again, by convexity, is a sphere (two points when the mirrors are 1-dimensional).

In general, consider $\ell$ mirrors $\Lambda_{1}, \ldots, \Lambda_{\ell}$ such that the inclusions of iterated intersections $\Lambda_{12} \supset \Lambda_{123} \supset \ldots \supset \Lambda_{123 \ldots \ell}$ are all strict. Then each intersection is a totally geodesic submanifolds of codimension 1 in the previous intersection, and hence $\Lambda_{123 \ldots \ell}$ is a $(k-\ell)$ sphere. Also $\Lambda_{123 \ldots \ell}$ is the set fixed by all reflections $r_{i}$ with corresponding mirror $\Lambda_{i}$. This completes the proof of the first "bullet".

Now suppose $C$ has $\ell+1$ chamber faces, $F_{0}, \ldots, F_{\ell}$. Since the angle between any two faces is at most $\frac{1}{2} \pi$ it follows as in the original Cheeger-Gromoll case of the distance function to the full boundary $[\mathrm{Pe}]$, that the distance function on $C$ to one face, say $F_{0}$, is strictly concave (cf. [Wi1, Theorem 7], [GKi, Theorem 1.3], and [Wö, Corollary 3.2] for general Alexandrov spaces with boundary). In particular, there is a unique point $s_{0}$ in $C$ at maximal distance to $F_{0}$ (the soul point for $F_{0}$ ). It follows that $s_{0}$ is in the intersection of the remaining chamber faces ([GKi, Theorem 1.3] and [Wö, Lemma 3.4] for general Alexandrov spaces, noting that intersections of faces are extremal subsets). Moreover, by convexity of super-level sets, the distance function to $s_{0}$ on $C$ has no critical points. Using this and the basic fact that convex combination of "gradient-like vector fields" is 
"gradient like", one constructs a gradient-like vector field (the angle between it and any minimal geodesic to $s_{0}$ is larger than $\frac{1}{2} \pi$ ), which is radial near $s_{0}$ and gradient like also when restricted to the remaining faces intrinsically. In particular, $C$ is the cone on $F_{0}$ which in turn is isotopic to a small metric ball in $C$ of radius $\varepsilon$ centered at $s_{0}$. This also identifies $F_{0}$ with the boundary of this $\varepsilon$ ball, which via the exponential map is identified with the closure of a chamber in the unit sphere at $s_{0}$ corresponding to the reflections $r_{1}, \ldots, r_{\ell}$. The proof of the remaining three bullets now follows from this and from the corresponding fact for finite linear reflection groups applied to the unite sphere at $s_{0}$.

We point out that this proof is a special case of a general result about orbit spaces of positively curved manifolds due to Wilking [Wi1] (Theorem 7), related more directly to $\Sigma / W$ in our context. We have included it here not only to make the exposition more self contained, but also because it illuminates the particular structure we have here.

We now turn to the case where the section $\Sigma$ is a projective space. In this case, we will analyze the situation in its universal cover $\widetilde{\Sigma}$. Specifically, for each mirror $\Lambda$ in $\Sigma$ corresponding to a reflection $r$, we consider its lift $\tilde{\Lambda}$ to $\widetilde{\Sigma}$. As noted in the proof of Proposition 2.3 and in Remark 2.4, $r$ has two canonical lifts. One of them is a reflection $\tilde{r}$ in $\tilde{\Lambda}$, the other has two isolated fixed points and restricts to a on $\tilde{\Lambda}$. Here we define $\widetilde{W}$ to be the reflection group on $\widetilde{\Sigma}$ generated by all $\tilde{r}$, where we use all $r$ from $W$. Note that, by construction, any lifted mirror is preserved by a, and that a commutes with any element from $\widetilde{W}$. Combining this with the previous lemma one derives, whether $M$ is simply connected or not, the following lemma.

LEMMA 2.6. (Projective chamber) Let $\Sigma$ be a $k$-dimensional projective space and $\widetilde{\Sigma}$ be the universal cover with deck transformation $\mathrm{a}$. Then the following statements hold.

- Intersections of lifted mirrors are spheres invariant under a.

- The associated reflection group $\widetilde{\mathrm{W}}$ of $\mathrm{W}$ may or may not contain a, but in either case $\mathrm{W}=\langle\widetilde{\mathrm{W}}, \mathrm{a}\rangle /\langle\mathrm{a}\rangle$.

- Open chambers $c$ in $\Sigma$ are isometric to open chambers $\tilde{c}$ for $\widetilde{W}$,

- The closure $\widetilde{C}$ of an open chamber for $\widetilde{W}$ is a convex set in $\widetilde{\Sigma}$ having the union of chamber faces as boundary. Moreover, $C$ is obtained from $\widetilde{C}$ by identifying a orbits in the boundary.

- $\widetilde{C}$ has at most $k+1$ chamber faces, and the intersection of them all is $\operatorname{Fix}(\widetilde{\mathrm{W}})$.

- If $\widetilde{C}$ has $k+1$ chamber faces it is a $k$-simplex and $\operatorname{Fix}(\widetilde{W})=\varnothing$.

- If $\widetilde{C}$ has $\ell+1<k+1$ chamber faces it is a join of $\operatorname{Fix}(\widetilde{W})$ and an $\ell$ simplex.

Moreover, $\Sigma / \mathrm{W}=\widetilde{\Sigma} /\langle\widetilde{\mathrm{W}}, \mathrm{a}\rangle=(\widetilde{\Sigma} /\langle\widetilde{\mathrm{W}}\rangle) /\langle\mathrm{a}\rangle=\widetilde{C} /\langle\mathrm{a}\rangle$.

We assume from now on that $M$ is a positively curved simply connected polar Gmanifold, with G connected. 
The following result is proved more generally for singular polar foliations in [AT, Theorem 1.5] and [A, Theorem 1.1].

Theorem 2.7. (Alexandrino and Töben) Any non-trivial polar action on a simply connected manifold has no exceptional orbits, and its reflection group $\mathrm{W}$ is the whole polar group $\Pi$.

In the case of polar actions, it was also recently proved in [GZ] that in addition the chamber group is trivial. For the sake of the reader, we provide a simple direct proof in the case of positive curvature. In fact, the following result is pivotal for us.

Proposition 2.8. (Chamber group) The chamber group $\mathrm{W}_{c}$ of a simply connected, positively curved polar G-manifold $M$ is trivial, and hence $M^{*}=\Sigma^{*}=C$. Moreover, the following statements hold.

- If $\Sigma$ is a sphere, $C$ is a simplex and the fixed-point set $\Sigma^{\mathrm{W}}=\varnothing$, or $C$ is a join of $\Sigma^{\mathrm{W}}$ and a simplex.

- If $\Sigma$ is a projective space, $C$ is a simplex, $\mathrm{a} \in \widetilde{\mathrm{W}}$ and $\Sigma^{\mathrm{W}}$ is a subset of the vertices (possibly empty).

In either case, $\mathrm{W}$ acts simply transitive on the set of chambers.

Proof. Consider an open chamber $c$ and $\mathrm{W}_{c}$ acting on it. Note that, whether or not $\Sigma$ is a sphere or a projective space, $c$ is the union of compact closed locally convex subsets $C^{\varepsilon}$ (distance $\varepsilon$ or more to $C \backslash c$ ). By convexity, it is clear that the soul point (the common soul point $s$ for all $C^{\varepsilon}$ ) is fixed by $\mathrm{W}_{c}$ (one can also use the description of $c$ from the lemmas above). Since there are no exceptional orbits when $M$ is simply connected (cf. Theorem 2.7), this already is impossible unless $\mathbf{W}_{c}$ is trivial. From $\S 1$ we then know that $M^{*}=\Sigma^{*}$ is the closure $C$ of a chamber $c$. If $\Sigma$ is a sphere, Lemma 2.5 completes the proof.

Now, suppose that $\Sigma$ is a projective space. First note that a acts freely on the set of open chambers for $\widetilde{W}$. This follows from the simple fact that a interchanges the two connected components of the complement of any lifted mirror, and commutes with $\widetilde{W}$.

We now claim that $\Sigma^{\widetilde{\mathrm{W}}}=\varnothing$, and hence $\widetilde{C}$ (defined in Lemma 2.6) is a simplex. Indeed, if $\Sigma^{\widetilde{W}}$ is non-empty then clearly a $\notin \widetilde{\mathrm{W}}$. Moreover, the involution induced by a on $\widetilde{\Sigma} / \widetilde{W}=\widetilde{C}$ acts freely on $\Sigma^{\widetilde{W}}$ and preserves the boundary of $\widetilde{C}$. In particular, $\widetilde{C} /\langle$ a $\rangle$ will have interior metric singular points contradicting that it is $C$ by Lemma 2.6.

To complete the proof we now claim that $\mathrm{a} \in \widetilde{\mathrm{W}}$, and in particular that

$$
C=\Sigma / \mathrm{W}=\widetilde{\Sigma} / \widetilde{\mathrm{W}}=\widetilde{C} .
$$

If not, then $|\mathrm{W}|=|\widetilde{\mathrm{W}}|$ and a induces a non-trivial involution on $\widetilde{C}$ with $C=\widetilde{C} /\langle\mathrm{a}\rangle$. Such an involution will preserve the boundary of the simplex $\widetilde{C}$ taking faces to faces. As before, 
this will produce an interior metric singular point of $C$, unless the induced map by a is a reflection of the simplex. This, however, cannot happen since the fixed-point set of this involution would correspond to a chamber face of $C$ and hence a reflection in $\mathrm{W}$ whose lift to $\widetilde{\Sigma}$ had been omitted from $\widetilde{W}$.

Remark 2.9. Note that it follows from this that if $M^{G} \neq \varnothing$ and $\Sigma$ is a sphere, then $M^{\mathrm{G}}=\Sigma^{\mathrm{W}}$, since $\Sigma^{\mathrm{W}}$ is the most singular stratum in the orbit space $\Sigma / \mathrm{W}=M / \mathrm{G}$. In the next section we will see that conversely, if $\Sigma^{\mathrm{W}} \neq \varnothing$ and $\Sigma$ is a sphere, then $M^{\mathrm{G}} \neq \varnothing$ as well, and hence $M^{\mathrm{G}}=\Sigma^{\mathrm{W}}$ (cf. (3.6)).

The Coxeter group W (resp. $\widetilde{W}$ ) corresponding to the section being a sphere (resp. a projective space) admits a canonical representation, i.e., acts isometrically on the unit $k$-sphere $\mathbb{S}^{k}$ with orbit space $C^{\prime}$ having the same infinitesimal singularities (i.e., tangent cones) as $C$. As a consequence, we have the following result.

Corollary 2.10. Let $M$ be a simply connected, positively curved polar G-manifold. Then $M^{*}=\Sigma^{*}=C$ admits a metric of constant curvature, isometric to its linear model $C^{\prime}$.

Proof. From Proposition 2.8, we know that $\Sigma^{*}$ is the chamber $C$ for a Coxeter group $\mathrm{W}($ resp. $\widetilde{\mathrm{W}}$ ) acting on the $k$-sphere $\Sigma$ (resp. $\widetilde{\Sigma}$, when $\Sigma$ is a projective space). Moreover, as stated above, the same Coxeter group acts linearly on $\mathbb{S}^{k}$, with chambers $C^{\prime}$ having labels as $C$ and with the same tangent cones, determined by the corresponding isotropy groups and actions.

We only consider the case where $\Sigma$ is a sphere since the other case is analogous. Now fix a chamber $C$ with $\ell+1$ chamber faces, $F_{0}, \ldots, F_{\ell}$ in $\Sigma$ and the corresponding model chamber $C^{\prime} \subset \mathbb{S}^{k}$. As in the proof of Lemma 2.5, let $s_{0}$ be the point in $C$ at maximal distance to the face $F_{0}$. Now apply the isotropy group $W_{s_{0}}$ of the Coxeter group at $s_{0}$ to $C$ to obtain a $\mathrm{W}_{s_{0}}$ invariant convex subset $\mathrm{W}_{s_{0}}(C)$ of $\Sigma$ with $s_{0}$ in the interior, the point at maximal distance from the boundary $\partial\left(\mathrm{W}_{s_{0}}(C)\right)=\mathrm{W}_{s_{0}}\left(F_{0}\right)$ of $\mathrm{W}_{s_{0}}(C)$. As in the proof of Lemma 2.5, it follows that there is a $\mathrm{W}_{s_{0}}$ invariant smooth vector field on an open neighborhood of $\mathrm{W}_{s_{0}}(C)$ in $\Sigma$, which is radial near $s_{0}$ and gradient like on $\partial\left(\mathrm{W}_{s_{0}}(C)\right)$.

The same construction based on $C^{\prime}$ in $\mathbb{S}^{k}$ yields a $W_{s_{0}}$ invariant diffeomorphism of a neighborhood of $\mathrm{W}_{s_{0}}(C)$ in $\Sigma$ to a neighborhood of $\mathrm{W}_{s_{0}}\left(C^{\prime}\right)$ in $\mathbb{S}^{k}$. After a suitable reparametrization of one of the vector fields using transversality if needed, the restriction yields the desired diffeomorphism from $C$ to $C^{\prime}$.

We are now ready to establish the main result of this section.

TheOREM 2.11. (Coxeter section) Let $M$ be a simply connected, positively curved polar manifold. Then the action of the polar group $\mathrm{W}$ of a section $\Sigma$ is equivariantly 
diffeomorphic to a linear action of $\mathrm{W}$. In fact, $\Sigma$ admits a $\mathrm{W}$ invariant metric of constant curvature.

Proof. Choose a constant curvature metric on $\Sigma^{*}$ as above. We now claim that this metric comes from a W invariant metric on $\Sigma$ with constant curvature. To see this, all we have to do is to lift the metric locally near any point of the orbit space to any point mapping to it by the orbit map. This however is clear. Since the lifted metrics obtained in this way agree on overlaps, we are done.

Remark 2.12. We point out that our conclusions in the theorem above carry over to the general context of a positively curved manifold with a non-trivial isometric reflection group action. The manifold together with the action of the reflection group is then equivariantly diffeomorphic to a sphere or a real projective space with a linear action by a finite Coxeter group ( $\mathbb{Z}_{2}$ ineffective in the latter case).

The following definition is now natural.

Definition 2.13. We say that a simply connected, positively curved polar G-manifold $M$ is reducible if the action by the Coxeter group $\mathrm{W}$ on $\Sigma$ is reducible.

In particular it follows that $W$, or $\widetilde{W}$, is an irreducible Coxeter system group when $(M, \mathrm{G})$ is irreducible; Example $1.1(3)$ shows that the converse is false. Also an action with a non-trivial fixed-point set is reducible. In the case of irreducible actions, all the types $\mathrm{A}_{n}, \mathrm{C}_{n}, \mathrm{D}_{n}, \mathrm{E}_{6}, \mathrm{E}_{7}, \mathrm{E}_{8}$ and $\mathrm{F}_{4}$ are of course possible when the section is a sphere, but we note that due to the chamber group proposition above, not all of them are possible when the section is a projective space.

We remark that in the literature the notion hyperpolar is used for a polar manifold with flat sections. Following [GZ], we say that a polar manifold is a polar space form if its sections have constant curvature. According to the sign of the curvature of the sections, one then says that the polar space form has spherical, euclidean or hyperbolic type. Using this language, a partition of unity argument as in [GZ, Theorem 3.3], or the main result of $[\mathrm{M}]$, now yields the following corollary in our case.

Corollary 2.14. (Polar space form) A simply connected, positively curved polar G manifold $M$ admits the structure of a polar spherical space form with the same sections.

It should be noted that $M$ with such a polar space form structure typically has curvatures of both signs. In general, a highly non-trivial result of $[\mathrm{M}]$ asserts that any metric on a section of any polar G-manifold invariant under the polar group extends to a G-invariant metric on the ambient manifold with the same section. 


\section{The chamber system and primitivity}

Based on the chamber group proposition (Proposition 2.8), recall from $\S 1$ that there are two natural chamber systems $\mathscr{C}(\Sigma, \mathrm{W}),($ resp. $\mathscr{C}(M, \mathrm{G}))$ associated with any polar action of a connected compact Lie group $\mathrm{G}$ on a simply connected, positively curved manifold $M$ with section $\Sigma$ and polar group $\mathrm{W}$. Throughout the rest of the paper $(M, \mathrm{G})$ is such a polar pair.

Our primary purpose in this section is to analyze $\mathscr{C}(M, \mathrm{G})$ further and thereby derive essential properties about such general actions. In particular, we will show that it is a connected chamber system (the crucial starting point for our subsequent investigation of irreducible actions), and use this to show that $\mathrm{G}$ is generated by the face isotropy groups of any fixed chamber $C \subset \Sigma$ (an essential ingredient in our investigation of reducible actions).

When the chambers are spherical simplices, we observe that all proper residues of the chamber system can be described via slice representations of corresponding isotropy groups. This allows us to invoke a celebrated result of Tits [Ti2], implying that in most cases the so-called universal cover of our chamber system is a building.

From the description of the chamber system $\mathscr{C}(M, \mathrm{G})=\bigcup_{g \in \mathrm{G}} g C$, we first note that all chambers are isometric when equipped with the induced length-space metric from $M$. This induces a natural length-space metric on each path-connected component of $\mathscr{C}(M, \mathrm{G})$. A fundamental theorem due to Wilking [Wi2] asserts in particular that the dual foliation associated with the orbits of an isometric group action on a positively curved manifold has only one leaf. It is an immediate consequence of this result that

- $\mathscr{C}(M, \mathrm{G})$ has only one component.

There is an equivalent length metric on $\mathscr{C}(M, \mathrm{G})$ obtained by using a polar space form metric on $M$ (cf. Corollary 2.14) in the construction above. We will refer to the corresponding topology as the thin topology on $\mathscr{C}(M, \mathrm{G})$. (Since $M$ is the union of its chambers, we can also think of it as $M$ being equipped with this metric and topology.)

From now on, we will always use the thin length metric on $\mathscr{C}(M, \mathrm{G})$ induced from a constant curvature-1 metric on a section. In particular, note that each chamber $C$ is either a (spherical) $k$-simplex $\Delta^{k}$, or else the spherical join $\mathbb{S}^{k-\ell-1} * \Delta^{\ell}$ of the $(k-\ell-1)$ sphere and a spherical $\ell$-simplex. In either case, the chambers in a fixed section $\Sigma$ tile the section, which is either $\mathbb{R} \mathbb{P}^{k}$ or $\mathbb{S}^{k}$. Moreover, by construction, $G$ preserves the labeling of all "vertices", "edges", ..., "faces", i.e., of all 0-, 1-, ..., $(k-1)$-simplices, when $C=\Delta^{k}$ is a simplex. In the special case where the chamber is not a simplex, i.e., $C=\mathbb{S}^{k-\ell-1} * \Delta^{\ell}$, by a vertex, or 0 -simplex, of the chamber $C$ we mean a set of the type $\mathbb{S}^{k-\ell-1} *\{v\}$, where $v$ is a vertex of the simplex $\Delta^{\ell}$, and similarly for edges, ..., faces. We label the set $\mathbb{S}^{k-\ell-1} \subset C$ as the $(-1)$-simplex of the chamber $C$. In either case, we note that the 
intersection of any two chambers in $M$ is either empty or else a common "subsimplex" in this sense, allowing in particular the intersection to be a "(-1)-simplex".

From the fact that $\mathscr{C}(M, \mathrm{G})$, with the thin topology, is connected, we get the following essential property.

TheOREM 3.1. (Connectivity) Let $M$ be a simply connected, positively curved polar $\mathrm{G}$-manifold. Then the associated chamber system $\mathscr{C}(M ; \mathrm{G})$ is connected, i.e., any two chambers are connected by a gallery.

Proof. We will prove this by induction on $\operatorname{dim} M^{*}=k$ using that $\mathscr{C}(M ; \mathrm{G})$ is path connected. For simplicity, we first present the proof in the typical case where the chamber $C$ is a simplex $\Delta^{k}$. A simple modification yields the general statement.

Let $C$ and $C^{\prime}$ be two chambers of $\mathscr{C}(M ; \mathrm{G})$. Using [Wi2] join two interior points of $C$ and $C^{\prime}$ by a piecewise smooth horizontal curve, i.e., at any point both one-sided derivatives of the curve are perpendicular to the $G$ orbit at the point. In our case, it is clear that we can choose a horizontal curve $\gamma:[0,1] \rightarrow M$, and $0=t_{0}<t_{1}<\ldots<t_{k+1}=1$ such that $\gamma_{-}\left(t_{i}, t_{i+1}\right)$ is a geodesic, or a once-broken geodesic in the interior of a chamber $C_{i}$ relative to the thin metric on $\mathscr{C}(M ; \mathrm{G})$, where $C_{0}=C, C_{k}=C^{\prime}$ and all $C_{i}$ are different. Moreover, $\gamma$ can be chosen so that each of the possibly non-smooth points $\gamma\left(t_{i}\right), i=1, \ldots, k$ are all vertices. In addition, the one-sided derivatives $\gamma_{+}^{\prime}\left(t_{i}\right)$ and $-\gamma_{-}^{\prime}\left(t_{i}\right)$ of $\gamma$ at the vertices $\gamma\left(t_{i}\right)$ are interior points of two $(k-1)$-chamber simplices for the chamber complex $\mathscr{C}\left(\mathbb{S}_{\gamma\left(t_{i}\right)}^{\perp} ; \mathrm{G}_{\gamma\left(t_{i}\right)}\right)$ of the slice representation of the isotropy group $\mathrm{G}_{\gamma\left(t_{i}\right)}$. By induction, these simplices can be joined by a gallery in $\mathscr{C}\left(\mathbb{S}_{\gamma\left(t_{i}\right)}^{\perp} ; \mathrm{G}_{\gamma\left(t_{i}\right)}\right)$. Filling in the corresponding gallery in $\mathscr{C}(M ; \mathrm{G})$ at each $\gamma\left(t_{i}\right)$ now yields a gallery from $C$ to $C^{\prime}$.

To complete the proof, we need to establish the induction anchor in cohomogeneity 2. By the same reasoning as above, this follows from the claim that the chamber complex of a linear spherical cohomogeneity-1 action is connected. Since any horizontal curve provided by Wilkings theorem in this case is a piecewise horizontal geodesic up to parametrization, such a curve already constitutes the desired gallery.

The modification needed to cover the case where the chambers are joins with a non-empty sphere can be explained as follows: As in the simplex case one may choose a piecewise horizontal geodesic $\gamma$, so that each of the possibly non-smooth points points $\gamma\left(t_{i}\right), i=1, \ldots k$, are most singular, i.e., in this case $(-1)$-simplex points. The remaining part of the proof follows the same path.

The Coxeter section theorem (Theorem 2.11) and the connectivity theorem above are the two crucial properties derived using positive curvature. We note that there is no reason for the chamber system of a simply connected polar space form of spherical type to be connected. However, the manifolds we actually classify in higher cohomogeneities 
in this paper are the chamber connected polar spherical space forms, i.e.,

- simply connected polar space forms $(M, \mathrm{G})$ of spherical type, with

- connected associated chamber system, $\mathscr{C}(M ; \mathrm{G})$.

In addition, this generality is important for the proof because G-invariant polar submanifolds of a positively curved polar manifold are typically not positively curved (cf. $\S 6$, proof of Hopf lemma).

The two assumptions above will be applied throughout the rest of the paper.

Using connectivity, we derive the following simple but powerful tool.

Theorem 3.2. (Primitivity) The group $\mathrm{G}$ is generated by the (identity components of the) face isotropy groups of any fixed chamber.

Proof. Fix a chamber $C_{0}$ and consider any other chamber $\mathrm{g} C_{0}, \mathrm{~g} \in \mathrm{G}$. Using the results above, let $\Gamma=\left(C_{0}, \ldots, C_{k}\right)$ be a gallery of type $i_{1} i_{2} \ldots i_{k}$, where $C_{k}=\mathrm{g} C_{0}$. By definition, note that any $C_{n}$ is obtained from $C_{n-1}$ by applying an element $g_{i_{n}}$ of the isotropy group for the common face $i_{n}$ of $C_{n}$ and $C_{n-1}$ to $C_{n-1}$, i.e., $C_{n}=\mathrm{g}_{i_{n}} C_{n-1}$. From this it follows that $C_{k}=\mathrm{g} C_{0}=\mathrm{g}_{i_{k}} \mathrm{~g}_{i_{k-1}} \ldots \mathrm{g}_{i_{1}} C_{0}$, and hence $\mathrm{g}=\mathrm{g}_{i_{k}} \mathrm{~g}_{i_{k-1}} \ldots \mathrm{g}_{i_{1}}$ after modifying $g_{i_{1}}$ with an element of the stabilizer of the chamber $C_{0}$, if necessary.

Now each $g_{i_{n}}$ is a conjugate of an element of the isotropy group corresponding to the face $i_{n}$ by the previous element. So, in other words, $\mathrm{g}_{i_{k}}=\left[\mathrm{g}_{i_{k-1}} \ldots \mathrm{g}_{i_{1}}\right] h_{i_{k}}\left[\mathrm{~g}_{i_{k-1}} \ldots \mathrm{g}_{i_{1}}\right]^{-1}$, and hence $\mathrm{g}=\left[\mathrm{g}_{i_{k-1}} \ldots \mathrm{g}_{i_{1}}\right] \mathrm{h}_{i_{k}}\left[\mathrm{~g}_{i_{k-1}} \ldots \mathrm{g}_{i_{1}}\right]^{-1} \mathrm{~g}_{i_{k-1}} \ldots \mathrm{g}_{i_{1}}=\left[\mathrm{g}_{i_{k-1}} \ldots \mathrm{g}_{i_{1}}\right] \mathrm{h}_{i_{k}}$, where $\mathrm{h}_{i_{k}}$ is in the isotropy group with face $i_{k}$ of $C_{0}$.

Proceeding in this way, we see that $\mathrm{g}=\mathrm{h}_{i_{1}} \mathrm{~h}_{i_{2}} \ldots . . \mathrm{h}_{i_{k}}$, where also $\mathrm{h}_{i_{1}}=\mathrm{g}_{i_{1}}$, as claimed. The claim about identity components of the face isotropy groups follows, since in fact they act transitively on the normal spheres of their orbit strata (these spheres are connected).

Remark 3.3. The description of galleries used in the proof above is very useful. In fact, a gallery starting at $C$ of type $i_{1} i_{2} \ldots i_{k}$ is given by a word $\mathrm{h}_{i_{1}} \mathrm{~h}_{i_{2}} \ldots \mathrm{h}_{i_{k}}$ in elements of the isotropy groups $\mathrm{G}_{i_{j}}$ corresponding to the $i_{j}$-faces of $C$. Note that each $\mathrm{G}_{i_{j}}$ acts transitively on the normal sphere to the corresponding orbit stratum, i.e., the $i_{j}$ residue of $C$ is in one-to-one correspondence with this normal sphere. For this reason, we say that a gallery $\Gamma_{f}=\left(C_{0}, \ldots, C_{k}\right)$ of type $f=i_{1} i_{2} \ldots i_{k}$ is obtained from $C_{0}$ by repeatedly folding it along the faces using the face isotropy groups $\mathrm{G}_{i_{1}}, \mathrm{G}_{i_{2}}, \ldots, \mathrm{G}_{i_{k}}$.

Remark 3.4. Note that this also immediately implies that $\mathrm{G}$ is generated by any two vertex isotropy groups.

Remark 3.5. We also observe that, in complete generality, our chamber system $\mathscr{C}(M ; \mathrm{G})$ associated with a polar $\mathrm{G}$ action on a simply connected manifold $M$ is a ho- 
mogeneous chamber system of a type described in Ronan's book [R]. Specifically, the following holds:

- the chamber system $\mathscr{C}(M ; \mathrm{G})$ is the left $\operatorname{coset} \mathrm{G} / \mathrm{H}$ (the principal orbit) with the following adjacency relation;

- two chambers $g \mathrm{H}$ and $g^{\prime} \mathrm{H}$ are $i$-adjacent if and only if $g \mathrm{G}_{i}=g^{\prime} \mathrm{G}_{i}$, where $\mathrm{H}$ is the principal isotropy group, and the $\mathrm{G}_{i}$ for $i \in I$ are the face isotropy groups of a fixed chamber.

Note that $\mathscr{C}(M ; \mathrm{G})$ is connected if and only if the polar $\mathrm{G}$-action is primitive, i.e., by definition, $G$ is generated by the face isotropy groups.

As promised, we can use the above connectedness to prove the fixed-point claim from the previous section.

Proposition 3.6. Let $M$ be a simply connected, positively curved polar G-manifold with spherical section $\Sigma$ and polar group $\mathrm{W}$. Then $M^{\mathrm{G}}=\Sigma^{\mathrm{W}}$, and in particular

$$
\operatorname{rk}(\mathrm{W})=\operatorname{dim} \Sigma^{*}+1-\operatorname{dim} M^{\mathrm{G}} .
$$

Proof. As obviously $M^{\mathrm{G}} \subset \Sigma^{\mathrm{W}}$, and equality has been proved in the previous section if $M^{G}$ is non-empty, it remains to prove that $M^{\mathrm{G}} \neq \varnothing$ as long as $\Sigma^{\mathrm{W}}$ is non-empty (cf. Remark 2.9).

By assumption,

$$
M^{*}=\Sigma^{*}=C=\Sigma^{\mathrm{W}} * \Delta^{\ell}=\mathbb{S}^{k-\ell-1} * \Delta^{\ell} .
$$

Since all G-orbits corresponding to $\Sigma^{\mathrm{W}}=\mathbb{S}^{k-\ell-1}$, are of the same type (corresponding to the most singular stratum of the orbit space) and are perpendicular to the section $\Sigma$, it suffices to see that $\Sigma^{\mathrm{W}}$ is preserved by $\mathrm{G}$.

Pick any $\mathrm{g} \in \mathrm{G}$ and join the chamber $\mathrm{g} C$ to $C$ with a gallery. As any two consecutive chambers in a gallery have a common "face" and thereby the same "(-1)-simplex", i.e., the same fixed-point set for the respective Weyl groups, it follows that also $\mathrm{g} C$ has the same "(-1)-simplex", which however is $\mathrm{g} \Sigma^{\mathrm{W}}$.

Example 3.7. Here are examples showing that the conclusion above may fail in cohomogeneity 1 , as well as when the section is a projective space.

(1) Let $M=\mathbb{C P}^{n}=\mathrm{SU}(n+1) / \mathrm{U}(n)$. Then $\mathrm{G}=\mathrm{U}(n)$ acts by cohomogeneity 1 with one fixed point. However, its polar group is $\mathbb{Z}_{2}$ acting on a section $\mathbb{S}^{1}$ with two fixed points.

(2) The obvious polar $\mathrm{G}=\mathrm{U}(1) \times \mathrm{U}(1) \times \mathrm{U}(n)$ representation on $\mathbb{C}^{n+2}=\mathbb{C}+\mathbb{C}+\mathbb{C}^{n}$ descends to a polar action on $\mathbb{C P}^{n+1}$ with two fixed points (corresponding to the two $\mathbb{C}$ summands). Its section is $\mathbb{R} \mathbb{P}^{2}$ with $\mathbb{R P}^{2} / \mathrm{W}=\mathbb{C P}^{n+1} / \mathrm{G}$ a right-angled spherical triangle. In particular, its Weyl group must necessarily have three fixed points. 
The case where the orbit space $M^{*}=\Sigma^{*}=C$ is not a simplex, i.e., by Proposition 2.8 it is a join of a sphere with a simplex (in particular $M^{\mathrm{G}} \neq \varnothing$ ), will be dealt with in $\S 6$.

We now point out some simple but crucial strong local properties of the chamber system $\mathscr{C}(M, \mathrm{G})$ of a positively curved, simply connected polar manifold in all remaining cases, i.e., when the orbit space is a simplex.

Say $\mathbf{M}=\left(m_{i j}\right)_{i, j \in I}$ is the Coxeter matrix of the reflection group $\mathbf{W}$ of the section $\Sigma$ if it is a sphere, or else of the reflection group $\widetilde{W}$ on the universal cover $\widetilde{\Sigma}$. In the latter case, any word in the generators $r_{i}$ of $W$ whose lift is the antipodal map in $\widetilde{W}$ is a non-Coxeter relation in $\mathrm{W}$, and must necessarily involve all generators of $\mathrm{W}$. For any fixed proper subset $J \subset I$, let $\mathrm{M}_{J}$ denote the submatrix of $\mathrm{M}$ with entries $m_{i j}, i, j \in J$. Correspondingly, we let $\mathrm{W}_{J}$ denote the subgroup of $\mathrm{W}$ generated by $\mathrm{r}_{i}, i \in J$. It is well known that the subgroup $\mathrm{W}_{J}$ of $\mathrm{W}$, as well as of $\widetilde{\mathrm{W}}$, is a Coxeter group of type $\mathrm{M}_{J}$.

Recall that, by definition, a chamber system $\mathscr{C}$ over $I$ has type $\mathrm{M}$ if all $\{i, j\}$ residues, $i, j \in I$, are so-called generalized $m_{i j}$-gons (cf. [R]).

For any chamber $C$, consider $C_{J}:=\bigcap_{i \in J} C_{i}$, where $C_{i}$ is the $i$-face of $C$. For an interior point $p \in C_{J}$, let $\mathbb{S}_{p, J}^{\perp}$ denote the unit sphere normal to the orbit stratum of $\mathrm{G} p$ at $p$, i.e., $\mathbb{S}_{p, J}^{\perp}$ is the sphere in the normal space to the orbit perpendicular to the fixed-point subspace of $\mathrm{G}_{p}$. The following result is now evident (see, e.g., Remark 3.3).

Lemma 3.8. (Residue) The J-residue of $\mathscr{C}$ and $\mathscr{C}\left(\mathbb{S}_{p, J}^{\perp}, \mathrm{G}_{p}\right)$, for any $p \in C_{J}$, are isomorphic as chamber systems of type $\mathrm{M}_{J}$.

Recall that a chamber system $\mathcal{B}$ over $I$ is called a building of type $\mathrm{M}=\left(m_{i j}\right), i, j \in I$, if each chamber is $i$-adjacent to at least one other chamber, and there is a $\mathrm{W}(\mathrm{M})$-valued "distance function"

$$
\delta: \mathcal{B} \times \mathcal{B} \longrightarrow \mathrm{W},
$$

with the property that $\delta(x, y)=\mathrm{w} \in \mathrm{W}$ if and only if the types of minimal galleries between $x$ and $y$ coincide with the types of minimal galleries in the Coxeter complex $\mathscr{C}(\Sigma, \mathrm{W})=: \mathcal{W}$ from 1 to $w$.

The Coxeter complex $\mathcal{W}$ is itself a building with $\delta(\mathrm{u}, \mathrm{v})=\mathrm{u}^{-1} \mathrm{v}$. We call "isometric" images of $\mathcal{W}$ in $\mathcal{B}$ apartments in $\mathcal{B}$. Another example of central importance to us is the following.

Example 3.9. (Polar representations) The chamber system, $\mathcal{B}=\mathscr{C}(\mathbb{S}, \mathrm{K})$ associated with the restriction of a polar representation of a compact Lie group $\mathrm{K}$ to the unit sphere $\mathbb{S}$ (without fixed points) is a fundamental example of a (spherical) Tits building (see [Ti1] and $[\mathrm{Dad}])$. 
Remark 3.10. (Basic building properties) In a building $\mathcal{B}$, the following properties are basic and used repeatedly in the next sections.

- (Connectedness) Any two chambers $x$ and $y$ are joined by a minimal gallery $\Gamma_{f}$, which in turn is contained in an apartment $A$.

- (Uniqueness) A minimal gallery from $x$ to $y$ is uniquely determined by its type.

- (Convexity) If $x$ and $y$ are chambers in an apartment $A$, every minimal gallery from $x$ to $y$ is contained in $A$.

- (Homotopy) If $\Gamma$ is a gallery from $x$ to $y$ of type $f$ (not necessarily minimal), and $f \simeq g$ (see below), then there is a gallery of type $g$ from $x$ to $y$.

- A gallery of type $f$ is minimal if and only if $f=i_{1} \ldots i_{m}$ is a so-called reduced word, or equivalently $\mathrm{w}=\mathrm{r}_{f}:=\mathrm{r}_{i_{1}} \ldots \mathrm{r}_{i_{m}}$ cannot be expressed as $\mathrm{r}_{g}$ for $g$ a shorter word.

Since the slice representation of each isotropy group $G_{p}$ is polar, Example 3.9 and the residue lemma (Lemma 3.8) imply the following result.

Proposition 3.11. For any proper $J \subset I$, any $J$-residue in the chamber system $\mathscr{C}(M, \mathrm{G})$ is a spherical building of type $\mathrm{M}_{J}$.

By invoking the following corollary of a profound result of Tits [Ti2, Corollary 3 in $\S 5.3]$ (cf. also [R, Theorem 4.9]), we get the following theorem.

THEOREM 3.12. (Tits) The universal Tits cover $\tilde{\mathscr{C}}$ of a (gallery-) connected chamber system $\mathscr{C}$ of (finite) type $\mathrm{M}$ over $I$ is a building if and only if all residues of rank 3 are covered by buildings.

We conclude the following result.

THEOREM 3.13. (Building cover) Let $M$ be a positively curved, simply connected polar G-manifold with orbit space a simplex of dimension at least 3 . Then the universal Tits cover $\tilde{\mathscr{C}}(M ; \mathrm{G})$ of the associated chamber system $\mathscr{C}(M ; \mathrm{G})$ is a spherical building.

Remark 3.14. The fact that all residues of rank at least 3 of the chamber system $\mathscr{C}(M ; \mathrm{G})$ are buildings implies that the universal Tits cover $\tilde{\mathscr{C}}(M ; \mathrm{G})$ can be viewed also as the usual topological universal cover of $\mathscr{C}(M ; \mathrm{G})$ equipped with the thin topology. For this reason we frequently simply refer to $\tilde{\mathscr{C}}(M ; \mathrm{G})$ as the universal cover of $\mathscr{C}(M ; \mathrm{G})$. In particular, the fundamental group $\pi$ of $\mathscr{C}(M ; G)$ acts freely by deck transformations on $\tilde{\mathscr{C}}(M ; \mathrm{G})$ equipped with the thin topology. It is a startling consequence of our main result Theorem 4.10 in $\S 4$ that $\pi$ in fact is either $\mathrm{S}^{1}$ or $\mathrm{S}^{3}$ with discrete topology when $\mathrm{M}$ has no isolated nodes and $\tilde{\mathscr{C}}(M ; \mathrm{G})$ is a building. Note also that $\pi$ acting freely on the set $\tilde{\mathscr{C}}(M ; \mathrm{G})$ of course is independent on topology.

Recall that the universal Tits cover is obtained via a notion of homotopies of galleries in analogy with the usual construction of a topological universal cover. 
Here two galleries, $\Gamma_{1} \Gamma_{0} \Gamma_{2}$ and $\Gamma_{1} \Gamma_{0}^{\prime} \Gamma_{2}$, in a chamber system $\mathscr{C}$ of type $\mathrm{M}$ over $I$ are said to be elementary homotopic if $\Gamma_{0}$ and $\Gamma_{0}^{\prime}$ are galleries in a rank-2 residue with the same extremities. A homotopy from a gallery $\Gamma$ to another one $\Gamma^{\prime}$ (with fixed extremities) is a finite sequence of elementary homotopies which transforms $\Gamma$ to $\Gamma^{\prime}$. When such a homotopy exists we write $\Gamma \simeq \Gamma^{\prime}$.

By construction, $\tilde{\mathscr{C}}$ as a set is a union of chambers, each chamber $\widetilde{C} \in \tilde{\mathscr{C}}$ being a homotopy class, $[\Gamma]=\left[C_{0}, \ldots, C_{m}\right]$ of galleries $\Gamma=\left(C_{0}, \ldots, C_{m}\right)$ from $\mathscr{C}$ starting at a fixed chamber $C_{0} \in \mathscr{C}$ and ending at $C_{m}=C \in \mathscr{C}$, and where the covering map $p: \tilde{\mathscr{C}} \rightarrow \mathscr{C}$ takes $\widetilde{C}$ to $C_{m}$. Also, the adjacency relation among chambers is defined as follows: $\widetilde{C}=\left[C_{0}, \ldots, C_{m}\right]$ is " $i$-adjacent" to $\widetilde{C}^{\prime}=\left[C_{0}, \ldots, C_{m-1}, C_{m}^{\prime}\right]$ when $C_{m}$ and $C_{m}^{\prime}$ are " $i$-adjacent", and to $\widetilde{C}^{\prime \prime}=\left[C_{0}, \ldots, C_{m}, C^{\prime \prime}\right]$ for other $i$ 's when $C_{m}$ and $C^{\prime \prime}$ are " $i$-adjacent". All other incidence relations follow from this, and the covering map $p$ preserves incidence relations. In this fashion, the covering map $p$ preserves faces, and hence all other types.

Note that, in a Coxeter complex, $\mathcal{W}$ galleries starting at 1 are in one-to-one correspondence with their types. Here one also uses the notion of strict homotopy, denoted $f \simeq g$, where the notion of an elementary homotopy above is replaced by the stronger notion of a strict elementary homotopy. Here a strict elementary homotopy is an alteration of a word of the form $f_{1} p(i, j) f_{2}$ to a word $f_{1} p(j, i) f_{2}$, where $p(i, j)$ is a word of the form $\ldots i j i j$ (with $m_{i j}$ letters and ending in $j$ ); e.g., if $m_{i j}=3, p(i, j)=j i j$; and $p(j, i)=i j i$. In particular, $f$ and $g$ have the same length if they are strictly homotopic (but not necessarily if they are just homotopic). Also, $\mathbf{r}_{f}=\mathrm{r}_{g}$ if $f$ and $g$ are strictly homotopic, but the converse is false, since one may have redundant letters; e.g, the words $f=f_{1} i i f_{2}$ and $g=f_{1} f_{2}$ are not strictly homotopic but $\mathbf{r}_{f}=\mathbf{r}_{g}$. A word $f$ is called reduced if it is not strictly homotopic to a word of the form $f_{1} i i f_{2}$.

Remark 3.15. Buildings are simplicial complexes, but our chambers systems $\mathscr{C}(M ; \mathrm{G})$ are frequently not. This is illustrated for example with the standard $\mathrm{T}^{2}$ action on $\mathbb{C P}^{2}$. Here all chambers are spherical right-angled 2-simplices, and they all have the same three vertices, the fixed points of $\mathrm{T}^{2}$. As the building cover theorem above shows, we do not need to assume $\mathscr{C}(M ; \mathrm{G})$ to be simplicial, when the rank of $\mathrm{M}$ is at least 4 . However, in the rank-3 case, where the building cover theorem says nothing, we do indeed need $\mathscr{C}(M ; \mathrm{G})$ to be simplicial in the irreducible cases, i.e., the cases of types $\mathrm{A}_{3}$ and $\mathrm{C}_{3}$. This will be proved in Theorem 5.1 and will allow us to use work of Tits on so-called geometries, i.e., chamber systems of type $\mathrm{M}$ whose underlying geometric realization is simplicial.

Remark 3.16. Equipped with the thin metric, our chamber system $\mathscr{C}(M, \mathrm{G})$ has the local structure of a CAT(1) space. This is of course true for its universal cover $\tilde{\mathscr{C}}(M, \mathrm{G})$ 
as well. In fact, when its dimension is at least 3 (corresponding to rank at least 4), it follows by work of Charney and Lytchak $[\mathrm{CL}]$, that in fact $\tilde{\mathscr{C}}(M, \mathrm{G})$ is a $\mathrm{CAT}(1)$ space and in fact a spherical building by their geometric characterization of buildings.

\section{Compact spherical buildings}

Throughout this section, we assume that the orbit space $M^{*}=C$ is a simplex, and that the universal cover $\tilde{\mathscr{C}}:=\tilde{\mathscr{C}}(M, \mathrm{G})$ of our base chamber system $\mathscr{C}:=\mathscr{C}(M, \mathrm{G})$ is a spherical building of rank at least 3 . In particular, $\tilde{\mathscr{C}}$ is also a simplicial complex and we use $p: \tilde{\mathscr{C}} \rightarrow \mathscr{C}$ to denote the covering map.

Our primary objective is to endow $\tilde{\mathscr{C}}$ with a natural topology inherited from the topology of $M$, in such a way that it becomes a compact spherical building in the sense of Burns and Spatzier [BS], where the extension by Grundhöfer, Kramer, Van Maldeghem and Weiss in $[\mathrm{GKMW}]$ is crucial for us. Our second objective is to analyze the fundamental group $\pi$ of $\mathscr{C}(M, \mathrm{G})$ and its action on the cover when $\tilde{\mathscr{C}}(M, \mathrm{G})$ is a compact spherical building. This in fact will imply Theorem A in the introduction in all cases except where $\mathrm{G}$ has fixed points, or where the Coxeter diagram for $\mathrm{M}$ either has isolated nodes or is of type $\mathrm{A}_{3}$ or $\mathrm{C}_{3}$.

$\S 5$ and $[\mathrm{FGT}]$ are devoted to the case where the Coxeter diagram of $\mathrm{M}$ is of type $\mathrm{A}_{3}$ or $\mathrm{C}_{3}$. In the special reducible cases where isolated nodes are present in the Coxeter diagram of $\mathrm{M}$ or $M^{\mathrm{G}} \neq \varnothing$, rather different arguments will be employed in $\S 6$ and $\S 7$.

We will write the set of vertices $\operatorname{Vert}(\tilde{\mathscr{C}})$ of a Tits building $\tilde{\mathscr{C}}$ as a disjoint union $\operatorname{Vert}(\tilde{\mathscr{C}})=\widetilde{V}_{1} \cup \ldots \cup \widetilde{V}_{k+1}$ over the vertices of the same cotype, where $k+1$ is the rank of M. The set of $r$-simplices of type $\left(i_{1}, \ldots, i_{r+1}\right)$ for $r \leqslant k$ will be denoted by $\tilde{\mathscr{C}}_{i_{1}, \ldots, i_{r+1}}$.

Recall that a compact (spherical) building, according to [BS], is a Tits building $\tilde{\mathscr{C}}$ with a Hausdorff topology on the set $\operatorname{Vert}(\tilde{\mathscr{C}})=\widetilde{V}_{1} \cup \ldots \cup \widetilde{V}_{k+1}$ of all vertices such that the set $\tilde{\mathscr{C}}_{i_{1}, \ldots, i_{r+1}}$ of all simplices of type $\left(i_{1}, \ldots, i_{r+1}\right)$ is closed in the product $\widetilde{V}_{i_{1}} \times \ldots \times \widetilde{V}_{i_{r+1}}$. With the induced topology on the $k$-simplices $\tilde{\mathscr{C}}_{1, \ldots, k+1}, \tilde{\mathscr{C}}$ is called compact, locally connected, infinite, metric if $\tilde{\mathscr{C}}_{1, \ldots, k+1}$ has the appropriate property.

It is the main result of [BS] that an infinite, irreducible, locally connected, compact, metric, topologically Moufang building of rank at least 2 is classical. Namely, it is a Tits building associated with a non-compact real semisimple Lie group via the following description (cf. also proof of Theorem 4.10).

Example 4.1. (Symmetric spaces and buildings) Let $\mathrm{U}$ be a connected non-compact real semisimple Lie group without center and $\mathrm{K} \subset \mathrm{U}$ a maximal compact subgroup (which is unique up to conjugation). The isometric action of $\mathrm{U}$ on the symmetric space $N=\mathrm{U} / \mathrm{K}$ of non-positive curvature induces a continuous action on the boundary at infinity, $\mathbb{S}_{\infty}$, 
with the same orbits as those of the subaction by $\mathrm{K}$. Here the action by $\mathrm{K}$ is topologically equivalent to the isotropy representation of $\mathrm{K}$ on the unit sphere $\mathbb{S}_{p}$ at $p \in N$ with $\mathrm{U}_{p}=\mathrm{K}$.

The isotropy representation of $\mathrm{K}=\mathrm{U}_{p}$ is polar with sections the tangent spaces of flats through $p \in N=\mathrm{U} / \mathrm{K}$. These flats at infinity are apartments of a (topological) building $\mathscr{C}(\mathrm{U})$ equivalent to $\mathscr{C}\left(\mathbb{S}_{p}, \mathrm{U}_{p}\right)$. One gets all apartments in the building in this fashion by letting $p$ go through all points of $N$. The group $\mathrm{U}$ is the identity component of the (topologiocal) automorphism group $\operatorname{Aut}_{\text {top }}(\mathscr{C}(\mathrm{U}))$ of the building.

An algebraic description of $\mathscr{C}(\mathrm{U})$ can be given via the set of all parabolic subgroups of $\mathrm{U}$. We think of $\mathscr{C}(\mathrm{U})$ as the set of parabolic subgroups of $\mathrm{U}$ with the following partial order: If $C_{1}, C_{2} \in \mathscr{C}(\mathrm{U})$, we call $C_{1}$ a face of $C_{2}$ and write $C_{1}<C_{2}$ if $C_{2} \subset C_{1}$. The chambers are the minimal parabolic subgroups. Let $W$ denote the Weyl group of the symmetric space $\mathrm{U} / \mathrm{K}$. We fix a minimal parabolic subgroup $\mathrm{B}$. The $\mathrm{W}$-valued metric $\delta$ in the definition of a building is then defined as follows: Given chambers $C=\mathrm{g} \mathrm{B}$ and $C^{\prime}=\mathrm{g}^{\prime} \mathrm{B}$, there is by the Bruhat decomposition a unique $w \in W$ such that $B g^{-1} g^{\prime} B=B w B$. We set $\delta\left(C, C^{\prime}\right)=$ w.

The correspondence between the geometric and algebraic description is that the isotropy groups under $\mathrm{U}$ of the chambers and their subsimplices at infinity are exactly the parabolic subgroups of $U$.

\section{The topology on $\tilde{\mathscr{C}}(M, \mathbf{G})$}

When considering $\mathscr{C}(M, \mathrm{G})$ as a set of chambers, each being a compact subset of the metric space $M, \mathscr{C}(M, \mathrm{G})$ is a compact metric space with the classical Hausdorff metric. Moreover, the same holds for the set of all galleries with any upper bound on the number of chambers. Since $\tilde{\mathscr{C}}(M, \mathrm{G})$ is a building of type $\mathrm{M}$ any two chambers can be connected by a gallery of length at most $\frac{1}{2}|\mathrm{~W}(\mathrm{M})|$.

Let us fix a chamber $\widetilde{C}_{0} \in \tilde{\mathscr{C}}$. For any fixed large positive integer $k \geqslant \frac{1}{2}|\mathrm{~W}(\mathrm{M})|, \varepsilon>0$ and any chamber $\widetilde{C} \in \tilde{\mathscr{C}}$, we let $B_{\varepsilon, k}(\widetilde{C})$ be the union of those chambers $\widetilde{C}^{\prime} \in \tilde{\mathscr{C}}$ for which there are (stuttering) galleries $\Gamma$ and $\Gamma^{\prime}$ of length at most $k$ starting at $\widetilde{C}_{0}$ and ending at $\widetilde{C}$ and $\widetilde{C}^{\prime}$, respectively, so that the (stuttering) galleries $p(\Gamma)$ and $p\left(\Gamma^{\prime}\right)$ in $\mathscr{C}$ are within Hausdorff distance $\varepsilon$ from one another in $M$. We will refer to the topology generated by these sets as the chamber topology on the building $\tilde{\mathscr{C}}$.

The geometric realization of the building $\tilde{\mathscr{C}}(M, \mathrm{G})$ is a simplicial complex $\tilde{\mathscr{S}}(M, \mathrm{G})$. We will show that this topology induces a topology on $\tilde{\mathscr{S}}(M, \mathrm{G})$ making it into a compact spherical building in the sense of Burns and Spatzier [BS].

The following lemma will be used repeatedly. 
LEMMA 4.2. (Homotopy control) Let $\Delta$ be a building of rank at least 3 . Then, for any $k$, there is a $C(k)$ with the following property: Any galleries $\Gamma$ and $\Gamma^{\prime}$ of lengths at most $k$ with the same extremities are homotopic by a homotopy consisting of at most $C(k)$ chambers.

Proof. Since any building of rank at least 3 is simply connected, $\Gamma$ and $\Gamma^{\prime}$ are homotopic. The remaining part of our claim is proved by induction on $k$, being trivially true for $k=1$.

If $\Gamma$ and $\Gamma^{\prime}$ are both minimal, the claim is a direct consequence of the convexity property in Remark 3.10. Similarly, if $\Gamma=\Gamma_{1} \Gamma_{0} \Gamma_{2}$ and $\Gamma^{\prime}=\Gamma_{1} \Gamma_{0}^{\prime} \Gamma_{2}$, where $\Gamma_{0}$ and $\Gamma_{0}^{\prime}$ are minimal (e.g., when there is a strict elementary homotopy from $\Gamma$ to $\Gamma^{\prime}$ ). In particular, by induction, it suffices to prove that a non-minimal $\Gamma$ is strictly homotopic to a $\Gamma^{\prime}$ via an a-priori bounded number of strict elementary homotopies and $\Gamma^{\prime}$ is homotopic to a shorter gallery within a uniformly bounded number of chambers.

Suppose that $\Gamma$ is not minimal of type $f$. We claim that $\Gamma$ is strictly homotopic to a gallery $\Gamma^{\prime}$ of type $f_{1} i i f_{2}$ through at most $\ell^{\ell}$ strictly elementary homotopies, where $\ell=|I|^{k}$. Indeed, the number of words of length at most $k$ is bounded above by $|I|^{k}$. Therefore, the non-circuit operations from a word of length at most $k$ to another one of length at most $k$ is bounded above by $\ell^{\ell}$.

Now, a gallery $\Gamma^{\prime}$ of type $f_{1} i i f_{2}$ from $x$ to $y$ is obviously homotopic to a shorter gallery of type either $f_{1} i f_{2}$ or $f_{1} f_{2}$, according to the chambers being $\Gamma_{1} C_{1} C_{2} C_{3} \Gamma_{2}$ (where $C_{1} \sim_{i} C_{2}$ and $C_{2} \sim_{i} C_{3}$ ) or $\Gamma_{1} C_{1} C_{2} C_{1} \Gamma_{2}$ (where $C_{1} \sim_{i} C_{2}$ ). Moreover, the homotopy can be realized in the longer gallery and so the number of chambers is bounded by the length $k$.

Remark 4.3. The proof of the above lemma gives an algorithm to construct a controlled homotopy between galleries with the same extremities in a building.

Proposition 4.4. With the chamber topology, $\tilde{\mathscr{C}}$ is a compact, separable and metrizable space.

Proof. By the Uryson characterization theorem for metrizable spaces, all we need to prove is that $\tilde{\mathscr{C}}$ is sequentially compact, separable and regular.

- (Sequential compactness) Any sequence $\left\{\widetilde{C}_{n}\right\}$ of chambers in $\tilde{\mathscr{C}}$ has a convergent subsequence.

For each $n$, let $\Gamma_{n}$ be a gallery of length at most $k$ joining $\widetilde{C}_{0}$ and $\widetilde{C}_{n}$. By compactness of $M$, the sequence $p\left(\Gamma_{n}\right)$ has a convergent subsequence in the Hausdorff metric topology with, as limit, a gallery $\bar{\Gamma}_{\infty}$ starting at $p\left(\widetilde{C}_{0}\right)$. By the unique homotopy lifting property (see [R, Lemma 4.4]), $\bar{\Gamma}_{\infty}$ can be uniquely lifted to a gallery, say $\Gamma_{\infty}$, starting at $\widetilde{C}_{0}$. By 
the definition of the chamber topology, we know that the corresponding subsequence of $\left\{\widetilde{C}_{n}\right\}$ converges to the end chamber of $\Gamma_{\infty}$.

- (Separability) We may choose a countable dense subset $Q_{i}$ of each face isotropy group $\mathrm{G}_{i}$, e.g. the rational points. The set of galleries starting at $\widetilde{C}_{0}$ of length at most $k$ obtained by the folding process described in Remark 3.3 using only elements from $Q_{i}$ is clearly dense in the set of all galleries starting at $\widetilde{C}_{0}$ of length at most $k$. By definition, the last chamber of these lifted galleries in $\tilde{\mathscr{C}}$ starting at $\widetilde{C}_{0}$ form a countable dense set in the chamber topology.

- (Regularity) We need to prove that, for a chamber $\widetilde{C}_{1}$ and a closed subset $B \subset \tilde{\mathscr{C}}$ in the complement of $\widetilde{C}_{1}$, there are two disjoint open sets $U$ and $V$ containing $\widetilde{C}_{1}$ and $B$, respectively.

If this is not the case, we find, for arbitrary large integers $n$, a chamber $\widetilde{C}_{n}^{\prime} \in$ $B_{1 / n, k}\left(\widetilde{C}_{1}\right) \cap B_{1 / n, k}(B)$. By the above, we know that the closed subset $B$ is sequentially compact. Therefore, a subsequence of $\widetilde{C}_{n}^{\prime}$ converges to some chamber $\widetilde{C}_{2} \in B$. Therefore, there are two pairs of sequences of galleries $\Gamma_{i, n}$ and $\Gamma_{i, n}^{\prime}, i=1,2$, such that the following holds: (a) $\Gamma_{i, n}$ starts at $\widetilde{C}_{0}$ and ends at $\widetilde{C}_{i}, i=1,2$; (b) $\Gamma_{i, n}^{\prime}$ starts at $\widetilde{C}_{0}$ and ends at $\widetilde{C}_{n}^{\prime}, i,=1,2 ;(\mathrm{c}) d_{H}\left(p\left(\Gamma_{i, n}\right), p\left(\Gamma_{i, n}^{\prime}\right)\right)<1 / n$. Since, for each $n, \Gamma_{1, n}^{\prime}$ and $\Gamma_{2, n}^{\prime}$ have the same extremities in the building $\tilde{\mathscr{C}}$, we have $\Gamma_{1, n}^{\prime} \simeq \Gamma_{2, n}^{\prime}$, and hence $p\left(\Gamma_{1, n}^{\prime}\right) \simeq p\left(\Gamma_{2, n}^{\prime}\right)$, by a homotopy $H_{n}^{\prime}$. By Lemma 4.2, we may assume that $H_{n}^{\prime}$ is composed of an a-priori bounded number of chambers independent of $n$. Taking convergent subsequences, we may assume that $p\left(\Gamma_{i, n}\right)$, as well as $p\left(\Gamma_{i, n}^{\prime}\right)$, converge to the same galleries $\bar{\Gamma}_{i, \infty}$, and that these are homotopic by a homotopy $H_{\infty}^{\prime}$. So, on the one hand, by the unique homotopy lifting property, $\bar{\Gamma}_{i, \infty}, i=1,2$, lift to galleries with the same end chamber in $\tilde{\mathscr{C}}$. On the other hand, they lift to galleries with end chamber $\widetilde{C}_{i}, i=1,2$, respectively. This yields a contradiction.

LEMma 4.5. (Independence) The chamber topology is independent of the choices of $\widetilde{C}_{0}$ and the parameter $k$.

Proof. Let us first prove the independence of $k$. If $k^{\prime}>k$, clearly $B_{\varepsilon, k}(\widetilde{C}) \subset B_{\varepsilon, k^{\prime}}(\widetilde{C})$. Consequently, it suffices to show that a $k^{\prime}$-convergent sequence of chambers $\left\{\widetilde{C}_{n}\right\}$ is also $k$-convergent. By assumption, there are galleries $\Gamma_{n}$ and $\Gamma^{n}$ in $\tilde{\mathscr{C}}$ of length at most $k^{\prime}$ starting at $\widetilde{C}_{0}$ and ending at $\widetilde{C}_{n}$ and $\widetilde{C}$, respectively, such that the projected galleries $p\left(\Gamma_{n}\right)$ and $p\left(\Gamma^{n}\right)$ Hausdorff converge to a gallery $\bar{\Gamma}_{\infty}$ (possibly stuttering) in $\mathscr{C}$. Again using Lemma 4.2, we see that the gallery $\Gamma_{n}$ is homotopic to a gallery $\Gamma_{n}^{\prime}$ of length at most $k$ by a homotopy $H_{n}$ with an a-priori bounded number of chambers. Note that $p\left(\Gamma_{n}^{\prime}\right)$ subsequentially converges to a gallery $\bar{\Gamma}_{\infty}^{\prime}=p\left(\Gamma_{\infty}^{\prime}\right)$, where $\Gamma_{\infty}^{\prime}$ is the subsequence limit of $\Gamma_{n}^{\prime}$. We may assume that the homotopies $p\left(H_{n}\right)$ also converge, and therefore 
we get a limit homotopy between the two limit galleries $\bar{\Gamma}_{\infty}$ and $\bar{\Gamma}_{\infty}^{\prime}$. By the homotopy uniqueness lifting property, once again we get that $\Gamma_{\infty}$ and $\Gamma_{\infty}^{\prime}$ have the same ending chambers $\widetilde{C}$. Therefore, $\left\{\widetilde{C}_{n}\right\}$ also $k$-converges to $\widetilde{C}$.

To see the independence of the choice of $\widetilde{C}_{0}$, join another chamber $\widetilde{C}_{0}^{\prime}$ to $\widetilde{C}_{0}$ with a fixed gallery $\Gamma_{0}$, and the claim follows from the independence of $k$ via concatenation with $\Gamma_{0}$ and its opposite.

We will now investigate the topology induced on the set of vertices from the chamber topology. That topology will in turn induce a topology on the geometric realization $|\tilde{\mathscr{S}}(M, \mathrm{G})|$ of the simplicial complex $\tilde{\mathscr{S}}(M, \mathrm{G})$ associated with the building referred to as the thick topology on $\tilde{\mathscr{C}}$ from now on. Assuming that our chamber system $\tilde{\mathscr{C}}$ has rank $k+1$, corresponding to cohomogeneity $k$, for any $i \in I=\{0, \ldots, k\}$ consider the set $\widetilde{V}_{i}$ of cotype- $i$ vertices in $\tilde{\mathscr{C}}$. Let $\pi_{i}: \tilde{\mathscr{C}} \rightarrow \widetilde{V}_{i}$ denote the obvious projection map. For each $i$, we equip $\widetilde{V}_{i}$ with the quotient topology.

Lemma 4.6. (Vertex space) For any $i \in I$, the projection $\pi_{i}: \tilde{\mathscr{C}} \rightarrow \widetilde{V}_{i}$ is an open map, and $\widetilde{V}_{i}$ is compact and Hausdorff. Moreover, for any $x \in \widetilde{V}_{i}$, the fiber $\pi_{i}^{-1}(x) \subset \tilde{\mathscr{C}}$ is the residue $\operatorname{Res}(x)$ in $\tilde{\mathscr{C}}$, which is compact, and the restriction of the covering map $p: \tilde{\mathscr{C}} \rightarrow \mathscr{C}$ to this residue is a homeomorphism to the residue $\operatorname{Res}(p(x))$ in $\mathscr{C}$.

Proof. We begin with a proof of the last claim. By construction of $\tilde{\mathscr{C}}, p$ provides an isomorphism between the residues as sub-buildings. We need to show that the chamber topology restricted to the residue Res $(x)$ coincides with the Hausdorff topology of $\operatorname{Res}(p(x))$ in the manifold $M$.

Since $\tilde{\mathscr{C}}$ and $\mathscr{C}$ are both compact and Hausdorff, and $p: \tilde{\mathscr{C}} \rightarrow \mathscr{C}$ obviously is continuous, it remains to check that $\operatorname{Res}(x)$ is closed in $\tilde{\mathscr{C}}$. Let $\left\{\widetilde{C}_{n}\right\}_{n \geqslant 1}$ be a sequence of chambers in $\operatorname{Res}(x)$ which converges in $\tilde{\mathscr{C}}$. Join a fixed chamber $\widetilde{C}_{0}$ to $\widetilde{C}_{1}$ by a gallery $\Gamma$. Using that the residues are buildings, join each $\widetilde{C}_{1}$ to $\widetilde{C}_{n}$ by a minimal gallery $\Gamma_{n}$ within the residue. A subsequence of the projections to $\mathscr{C}$ of the concatenated galleries clearly converges in the Hausdorff topology, and the end chamber of the lift of the limiting gallery is the limit of $\left\{\widetilde{C}_{n}\right\}_{n \geqslant 1}$, which as a consequence is in the residue.

To show that $\widetilde{V}_{i}$ is Hausdorff, it suffices to show that $\pi_{i}: \tilde{\mathscr{C}} \rightarrow \widetilde{V}_{i}$ is an open map and that the cotype- $i$ adjacency is a closed relation, i.e. the subset

$$
\left\{\left(\widetilde{C}, \widetilde{C}^{\prime}\right) \in \tilde{\mathscr{C}} \times \tilde{\mathscr{C}}: \widetilde{C} \text { and } \widetilde{C}^{\prime} \text { have common cotype- } i \text { vertices }\right\}
$$

is closed in the product topology. To show the latter, let $\left(\widetilde{C}_{n}, \widetilde{C}_{n}^{\prime}\right)$ be a sequence converging to $\left(\widetilde{C}, \widetilde{C}^{\prime}\right)$, where $\pi_{i}\left(\widetilde{C}_{n}\right)=\pi_{i}\left(\widetilde{C}_{n}^{\prime}\right)$. In particular, $C_{n}$ and $C_{n}^{\prime}$ share an $i$-vertex, and $\left(C_{n}, C_{n}^{\prime}\right)$ converges to $\left(C, C^{\prime}\right)$ in the Hausdorff topology of $M$. Join $\widetilde{C}_{n}$ to $\widetilde{C}_{n}^{\prime}$ by a minimal gallery $\Gamma_{n}^{i}$ in the $i$-residue and pick a subsequence if necessary so that the 
image galleries $p\left(\Gamma_{n}^{i}\right)$ in the residues in $M$ converge. Obviously, the limit gallery joins $C$ to $C^{\prime}$, and in particular, they share an $i$ vertex. It follows that $\widetilde{C}$ and $\widetilde{C}^{\prime}$ share the type- $i$ vertex.

Let us prove that $\pi_{i}$ is open. For this, we need to see that $\pi_{i}^{-1}\left(\pi_{i}(U)\right)$ is open in $\tilde{\mathscr{C}}$, where $U$ is the intersection of a finite number of the $B_{\varepsilon_{i}, k}\left(\widetilde{C}_{i}\right)$ 's. Pick a chamber $\widetilde{D}^{\prime} \in \pi_{i}^{-1}\left(\pi_{i}(U)\right)$, i.e. $\pi_{i}\left(\widetilde{D}^{\prime}\right)=\pi_{i}\left(\widetilde{C}^{\prime}\right)$ for some $\widetilde{C}^{\prime} \in U$. We need to find a neighborhood $U^{\prime}$ of $\widetilde{D}^{\prime}$ such that for any $\widetilde{D}^{\prime \prime} \in U^{\prime}$, there is a $\widetilde{C}^{\prime \prime} \in U$ with $\pi_{i}\left(D^{\prime \prime}\right)=\pi_{i}\left(C^{\prime \prime}\right)$.

Let $V$ be the intersection of a finite number of the $B_{\varepsilon_{j}, k}\left(\widetilde{C}_{j}\right)^{\prime}$ s such that $\widetilde{C}^{\prime} \in V \subset U$. Since $\widetilde{C}^{\prime}$ and $\widetilde{D}^{\prime}$ are cotype- $i$ adjacent, they are in the same cotype- $i$ residue (of some vertex), and they can be joined within this residue by a gallery $\Gamma$ explicitly obtained by folding (see Remark 3.3) $\widetilde{C}^{\prime}$ repeatedly along faces using face isotropy groups (fixing the cotype- $i$ vertex) in $M$ via $p: \tilde{\mathscr{C}} \rightarrow \mathscr{C}$. To complete the proof, the following observation suffices: Consider the chamber $C^{\prime}=p\left(\widetilde{C}^{\prime}\right)$ in $\mathscr{C}$. Any chamber Hausdorff close to $C^{\prime}$ is $\mathrm{g} C^{\prime}$ for some $\mathrm{g} \in \mathrm{G}$ close to $1 \in \mathrm{G}$, and $\mathrm{g} \Gamma$ is thus close to $\Gamma$. Therefore, this process and its inverse take a neighborhood of $\widetilde{C}^{\prime}$ to a neighborhood $\widetilde{D}^{\prime}$ and conversely, and the claim follows.

Now we are ready to prove the first of our main results in this section.

THEOREM 4.7. (Compact spherical building) The spherical building $\tilde{\mathscr{C}}(M, \mathrm{G})$ with the topology on the set of vertices induced by the thick topology on the chambers is a compact spherical building if its rank is at least 3 .

Proof. We have seen in Lemma 4.6 that the space $V_{1} \cup \ldots \cup V_{k+1}$ of vertices is Hausdorff. It is therefore left to show that the set $\tilde{\mathscr{C}}_{i_{1}, \ldots, i_{r+1}}$ of all simplices of type $\left(i_{1}, \ldots, i_{r+1}\right)$ is closed in the product $\widetilde{V}_{i_{1}} \times \ldots \times \widetilde{V}_{i_{r+1}}$. It follows from Proposition 4.4 and Lemma 4.6 that the product map $\prod_{j} \pi_{i_{j}}: \tilde{\mathscr{C}}_{i_{1}, \ldots, i_{r+1}} \rightarrow \widetilde{V}_{i_{1}} \times \ldots \times \widetilde{V}_{i_{r+1}}$ is continuous for any multi-index $i_{1}, \ldots, i_{r+1}$, and its image is a closed subset, which finishes the proof.

It is clear from what we have proved so far that the compact spherical building in Theorem 4.7 is an infinite compact metrizable building. We can now apply the main results of $[\mathrm{BS}]$, or rather its generalization in [GKMW], to compact spherical buildings that need not be locally connected.

THEOREM 4.8. (Classical building) Assume the compact spherical building $\tilde{\mathscr{C}}(M, \mathrm{G})$ has rank at least 3 and its associated Coxeter diagram has no isolated nodes. Then it is the building at infinity of a product $N$ of irreducible symmetric spaces of non-compact type of rank at least 2 . The topological automorphism group $\operatorname{Aut}_{\text {top }}(\tilde{\mathscr{C}})$ of the building $\tilde{\mathscr{C}}(M, \mathrm{G})$ is a real non-compact semisimple Lie group with finitely many connected components, and its identity component is isomorphic to the identity component of the isometry group of the symmetric space $N$. 
Proof. It follows from [GKMW, Theorem 1.2] that $\tilde{\mathscr{C}}(M, \mathrm{G})$ is the building at infinity of a product of irreducible symmetric spaces of rank at least 2 and a locally finite BruhatTits building of dimension at least 2. The building at infinity of the Bruhat-Tits building is totally disconnected and can therefore be excluded, since by Lemma 4.6 the vertex residues are locally connected compact spherical buildings. The claims about $\operatorname{Aut}_{\text {top }}(\tilde{\mathscr{C}})$ follow from [BS].

We now prove a general theorem about the lifted $\widetilde{\mathrm{G}}$-action and the free subaction of $\pi$ (cf. Remark 3.14) on the simplicial complex $\tilde{\mathscr{C}}(M, \mathrm{G})$ when equipped with the thick topology.

THEOREm 4.9. (Compact transformation group) Assume that the spherical building $\tilde{\mathscr{C}}(M, \mathrm{G})$ has rank at least 3 and is equipped with the thick topology. Then the deck transformation group $\pi$ with the compact open topology is a compact subgroup of the topological automorphism group $\operatorname{Aut}_{\text {top }}(\tilde{\mathscr{C}})$. Moreover, there is a compact subgroup $\widetilde{\mathrm{G}}$ of $\operatorname{Aut}_{\text {top }}(\tilde{\mathscr{C}})$, such that $\pi \subset \widetilde{\mathrm{G}}$ is a normal subgroup with quotient $\widetilde{\mathrm{G}} / \pi=\mathrm{G}$, whose action covers the $\mathrm{G}$-action on $\mathscr{C}$.

Proof. It is a simple consequence of the independence lemma (Lemma 4.5) that every element of $\pi$ is a homeomorphism with respect to the chamber and thick topologies. In particular, $\pi$ is a subgroup of the topological automorphism group $\operatorname{Aut}_{\text {top }}(\tilde{\mathscr{C}})$. We now prove that $\pi$ is a closed subgroup of $\operatorname{Aut}_{\text {top }}(\tilde{\mathscr{C}})$. Let $f_{n}$ be a sequence in $\pi$ that converges to $f$ in $\operatorname{Aut}_{\text {top }}(\tilde{\mathscr{C}})$ in the compact open topology. In particular, $f_{n}(\widetilde{C})$ converges to $f(\widetilde{C})$ in the chamber topology for every chamber $\widetilde{C} \in \tilde{\mathscr{C}}$. Notice that $p\left(f_{n}(\widetilde{C})\right)=p(\widetilde{C})$. Therefore, $p(f(\widetilde{C}))=p(\widetilde{C})$, and it follows that $f$ is in $\pi$. The compactness of $\pi$ follows, since the orbit of $\pi$ is compact and the action of $\pi$ is free.

It is well known that the $\mathrm{G}$-action on $\mathscr{C}$ lifts to a covering group $\widetilde{\mathrm{G}}$-action on $\tilde{\mathscr{C}}$, where $\widetilde{G}$ fits in an extension (see [R, Exercise 8 in Chapter 4$]$ )

$$
1 \longrightarrow \pi \longrightarrow \widetilde{\mathrm{G}} \longrightarrow \mathrm{G} \longrightarrow 1
$$

Once again, by the independence lemma, we see that $\widetilde{G}$ is a subgroup of $\operatorname{Aut}_{\text {top }}(\tilde{\mathscr{C}})$, and as above one can check that it is closed, and hence also compact, since both $\pi$ and G are.

Combining these results we have the following main result about polar manifolds of positive curvature.

THEOREM 4.10. Any polar action of a compact connected Lie group $\mathrm{G}$ on a simply connected, positively curved manifold $M$, whose associated chamber system is covered by a spherical building $\tilde{\mathscr{C}}$ of rank at least 3 and whose diagram $\mathrm{M}$ contains no isolated 
nodes, is equivariantly diffeomorphic to a polar action on a compact rank-1 symmetric space, other than the Cayley plane.

Proof. It follows from Theorem 4.8 that the simplicial complex $\tilde{\mathscr{S}}$ as a set with the thick topology is a sphere that we will denote by $\mathbb{S}$. The compact subgroup $\pi$ of $\operatorname{Aut}_{\text {top }}(\tilde{\mathscr{C}})$ is a Lie group, since $\operatorname{Aut}_{\text {top }}(\tilde{\mathscr{C}})$ is a Lie group by Theorem 4.8 .

We would like to show that $\pi$ is connected. We denote the identity component of $\pi$ by $\pi_{0}$. Clearly, $\pi_{0}$ acts freely on the sphere $\mathbb{S}$, and there is a covering $\mathbb{S} / \pi_{0} \rightarrow \mathbb{S} / \pi=M$ whose fiber has the same number of points as $\pi / \pi_{0}$. This is a contradiction, since $M$ is simply connected. It follows that $\pi=\pi_{0}$, and that both $\pi$ and $\widetilde{G}$ are compact and connected subgroups, i.e., Lie subgroups of the identity component $U$ of $\operatorname{Aut}_{\text {top }}(\tilde{\mathscr{C}})$. As a consequence, $\widetilde{G}$ has a fixed point in the symmetric space $U / K$, where $K$ is a maximal compact subgroup of the semisimple Lie group $U$. Therefore, up to conjugation, we may assume that $\widetilde{G} \subset K$ and it follows that the action by $\widetilde{G}$ is topologically equivalent to a linear polar action orbit equivalent to the isotropy representation of $\mathrm{K}$ on $\mathbb{S}$. Since the action of $\pi$ on $\mathbb{S}$ is both linear and free, $\pi$ is either $\{1\}, S^{1}$ or $S^{3}$ (cf. e.g. [Br]), and by representation theory the action is the Hopf action. It follows that $M$ is $\mathrm{G}$-equivariantly homeomorphic to the rank-1 symmetric space $\mathbb{S} / \pi$ with the linear polar action by $\mathrm{G}=\widetilde{\mathrm{G}} / \pi$.

To complete the proof, we note that the induced linear polar action on $\mathbb{S} / \pi$ by $\mathrm{G}=\widetilde{\mathrm{G}} / \pi$ has the same data, i.e., section, polar group, isotropy groups and their slice representations as the polar G-action on $M$. From the reconstruction theorem of [GZ], it follows that $(M, \mathrm{G})$ is smoothly equivalent to $(\mathbb{S} / \pi, \mathrm{G})$.

In view of the building cover theorem (Theorem 3.13), this takes care of all cases where $\mathrm{G}$ has no fixed points and $\mathrm{M}$ has no isolated nodes and rank at least 4 .

We conclude this section with another application of Theorem 4.10. As mentioned in the introduction, there are polar G-actions by $\mathrm{SU}(3) \mathrm{SU}(3)$ and $\mathrm{SO}(3) \mathrm{G}_{2}$ on $\mathbb{O P}^{2}$ (see $[\mathrm{PTh}]$ and $[\mathrm{GKo}])$ whose associated chamber systems $\mathscr{C}\left(\mathbb{O P}^{2}, \mathrm{G}\right)$ are of type $\mathrm{C}_{3}$. In particular we deduce from Theorem 4.10 the following result.

COROLlary 4.11. (Not a building) The universal covers of the chamber systems $\mathscr{C}\left(\mathbb{O P}^{2}, \mathrm{G}\right)$ associated with the polar actions on $\mathbb{O P}^{2}$ by $\mathrm{G}=\mathrm{SU}(3) \cdot \mathrm{SU}(3)$ or $\mathrm{G}=\mathrm{SO}(3) \cdot \mathrm{G}_{2}$ are simply connected chamber systems of type $\mathrm{C}_{3}$ that are not buildings.

Examples of simply connected chamber systems of type $C_{3}$ that are not buildings were discovered by Neumaier, and later but independently by Aschbacher. The examples of the chamber systems in Corollary 4.11 are new and follow also from [L] and [KL], as noted by them. These intriguing examples motivate the following interesting problems. 
Problem 4.12. (Cayley plane chamber system) Let $\tilde{\mathscr{C}}$ denote the universal cover of the chamber system $\mathscr{C}:=\mathscr{C}\left(\mathbb{O P}^{2} ; \mathrm{G}\right)$, where $\mathrm{G}$ is either $\mathrm{SU}(3) \cdot \mathrm{SU}(3)$ or $\mathrm{SO}(3) \cdot \mathrm{G}_{2}$.

(1) Is $\mathscr{C}$ itself simply connected?

(2) If $\mathscr{C}$ is not simply connected, does the section $\mathbb{R} \mathbb{P}^{2}$ lift to $\mathbb{S}^{2}$ in $\tilde{\mathscr{C}}$ ? What is its fundamental group, and what is $\tilde{\mathscr{C}}$ ?

\section{Irreducible chamber systems and Tits geometries of rank 3}

The purpose of this section is to develop and describe an alternative to the building cover theorem (Theorem 3.13) for irreducible polar actions of cohomogeneity 2, i.e., for rank-3 chamber systems $\mathscr{C}=\mathscr{C}(M, \mathrm{G})$, where $\mathrm{M}$ has no isolated nodes, or equivalently $\mathrm{M}$ is of type $\mathrm{A}_{3}$ or $\mathrm{C}_{3}$. In this case, any closed chamber $C$ of $\mathscr{C}$, or equivalently the $\mathrm{G}$-orbit space of $M$, is the spherical triangle with angles $\left\{\frac{1}{3} \pi, \frac{1}{2} \pi, \frac{1}{3} \pi\right\}$ in the $\mathrm{A}_{3}$ case, or $\left\{\frac{1}{4} \pi, \frac{1}{2} \pi, \frac{1}{3} \pi\right\}$ in the $\mathrm{C}_{3}$ case.

Our method is based on a construction of chamber system covers (corresponding to the principal bundle construction for polar manifolds in [GZ]), and on an axiomatic characterization due to Tits of buildings of irreducible type $M$, when the geometric realization $|\mathscr{C}|(\mathscr{C}$ with the thin topology) of the associated chamber system $\mathscr{C}$ is a simplicial complex. This characterization is given in terms of the incidence geometry associated with $\mathscr{C}$. Here, by definition,

- two vertices $x, y \in|\mathscr{C}|$ are incident, denoted by $x * y$, if and only if $x$ and $y$ are contained in a closed chamber of $|\mathscr{C}|$.

Clearly, the incidence relation (not an equivalence relation) is preserved by the action of $\mathrm{G}$ in our case.

To describe the needed characterization, and to prove that our chamber systems $\mathscr{C}(M, \mathrm{G})$ of type $\mathrm{A}_{3}$ or $\mathrm{C}_{3}$ are simplicial, we will use the following standard terminology:

- The shadow of a vertex $x$ on the set of vertices of type $i \in I$, denoted $\operatorname{Sh}_{i}(x)$, is the union of all vertices of type $i$ incident to $x$.

When $\mathrm{M}=\mathrm{C}_{3}$, we will use $q, r$ and $t$, respectively, to denote the vertices of a chamber $C$ at angles $\frac{1}{4} \pi, \frac{1}{2} \pi$ and $\frac{1}{3} \pi$, respectively, corresponding to the three nodes, from left to right, of the following $\mathrm{C}_{3}$-diagram:

The faces in $C$ opposite to $q, r$ and $t$ will be denoted by $\ell_{q}, \ell_{r}$ and $\ell_{t}$, respectively.

Following Tits [Ti2], we call the vertices of type $q, r$ and $t$, points, lines and planes, respectively. We denote by $Q, R$ and $T$ the set of points, lines and planes in $\mathscr{C}(M ; \mathrm{G})$, respectively. Notice that $\mathrm{G}$ acts transitively on $Q, R$ and $T$. 
Using this terminology we prove the following key result.

THEOREM 5.1. (Simplicial) The geometric realization $|\mathscr{C}(M, \mathrm{G})|$ of a chamber system $\mathscr{C}(M, \mathrm{G})$ of type $\mathrm{A}_{3}$ or $\mathrm{C}_{3}$ associated with a simply connected polar $\mathrm{G}$-manifold $M$ is simplicial.

Proof. Since the case of $A_{3}$ follows directly from a part of the proof of the $C_{3}$ case (cf. case (i) below), we only discuss the latter.

We claim that all we need to show is that vertices of different types are joined by at most one minimal geodesic. In particular, an edge is determined by its vertices. In fact, given this, we only need to prove that any chamber $C$ of $|\mathscr{C}(M, \mathrm{G})|$ is uniquely determined by its vertices. So suppose $C$ and $C^{\prime}$ are chambers with the same vertices. From the claim, they have the same edges as well. Now, by transitivity, there is a $\mathrm{g} \in \mathrm{G}$ with $\mathrm{g} C=C^{\prime}$. Since $\mathrm{g}$ fixes all vertices and edges of $C$, it is in the principal isotropy group of $C$, and hence $\mathrm{g} C=C$.

Case (i). One of the vertices is a plane.

For a plane $t \in T$, note that the shadow $\operatorname{Sh}_{Q}(t)\left(\right.$ resp. $\left.\mathrm{Sh}_{R}(t)\right)$ of $t$ in $Q$ (resp. $R$ ) is the homogeneous space $\mathrm{G}_{t} q=\mathrm{G}_{t} /\left(\mathrm{G}_{t} \cap \mathrm{G}_{q}\right.$ ), where $q \in \mathrm{Sh}_{Q}(t)$ (resp. $q \in \mathrm{Sh}_{R}(t)$ ). Moreover, the set of all edges containing $t$ and $q$ is the homogeneous space $\left(\mathrm{G}_{t} \cap \mathrm{G}_{q}\right) \ell_{r}=$ $\left(\mathrm{G}_{t} \cap \mathrm{G}_{q}\right) / \mathrm{G}_{\ell_{r}}$, where $\ell_{r}$ is a minimal reference geodesic connecting $t$ and $q$. It suffices to prove that $\mathrm{G}_{\ell_{r}}=\mathrm{G}_{t} \cap \mathrm{G}_{q}$.

Consider the fibration

$$
\frac{\mathrm{G}_{t} \cap \mathrm{G}_{q}}{\mathrm{G}_{\ell_{r}}} \longrightarrow \frac{\mathrm{G}_{t}}{\mathrm{G}_{\ell_{r}}} \longrightarrow \frac{\mathrm{G}_{t}}{\mathrm{G}_{t} \cap \mathrm{G}_{q}}
$$

Note that the base cannot be a point, since otherwise $\mathrm{G}_{t} \subset \mathrm{G}_{q}$, and so, by the primitivity, $\mathrm{G}=\left\langle\mathrm{G}_{t}, \mathrm{G}_{q}\right\rangle=\mathrm{G}_{q}$, and hence $\mathrm{G}$ would have fixed points. On the other hand, $\mathrm{G}_{t} / \mathrm{G}_{\ell_{r}}$ is the set of points (resp. planes) in a type- $\mathrm{A}_{2}$ geometry, associated with the slice representation at $t$, i.e., $\mathrm{G}_{t} / \mathrm{G}_{\ell_{r}}=\mathbb{P}^{2}(k)$, where $k$ is $\mathbb{R}, \mathbb{C}, \mathbb{H}$ or $\mathbb{O}$. In particular, $\mathrm{G}_{\ell_{r}}$ is a maximal subgroup of $\mathrm{G}_{t}$, and thus $\mathrm{G}_{\ell_{r}}=\mathrm{G}_{t} \cap \mathrm{G}_{q}$.

Case (ii). One of the vertices is a line.

Consider a chamber $C$ with sides $\ell_{t}, \ell_{r}$, and $\ell_{q}$, and suppose that $\ell_{t}^{\prime}$ is another minimal geodesic joining the vertices $r$ and $q$ of $C$. Since each singular isotropy group of the reducible slice representation of $G_{r}$ acts transitively on the other singular orbit, there is a $\mathrm{g} \in \mathrm{G}_{\ell_{q}} \subset \mathrm{G}_{r}$ with $\mathrm{g} \ell_{t}=\ell_{t}^{\prime}$. By (i), $\mathrm{g} C$ is a chamber with sides $\ell_{t}^{\prime}, \ell_{r}$ and $\ell_{q}$. But as $\mathrm{g}$ fixes $\ell_{r}$ and $\ell_{q}$, it is in the principal isotropy group of the slice representation of $\mathrm{G}_{t}$ and hence $\mathrm{g} C=C$. Thus $\ell_{t}^{\prime}=\ell_{t}$. 
Since by work of Tits [Ti2] (cf. Proposition 6), any $\mathrm{A}_{n}$-geometry is a building, we conclude as in Theorem 4.10 (with $\pi$ trivial) the following result.

COROLlary 5.2. A simply connected, positively curved polar $\mathrm{G}$-manifold of type $\mathrm{A}_{3}$ is equivariantly diffeomorphic to a polar G-representation on a sphere.

In the much more complicated and rich case where the chamber system $\mathscr{C}(M, \mathrm{G})$ is of type $\mathrm{C}_{3}$, our classification carried out in [FGT] hinges on an axiomatic characterization for a connected Tits geometry of type $C_{3}$ to be a building [Ti2].

For all but two such chamber systems, this Tits axiom is verified for a suitable cover of $\mathscr{C}(M, \mathrm{G})$, and the two exceptional cases are identified with the chamber systems for the two exceptional polar actions of type $C_{3}$ on the Cayley plane. As by Theorem 5.1 $\mathscr{C}(M, \mathrm{G})$ is simplicial, an alternative proof is offered in $[\mathrm{KL}]$.

\section{Reduction input and fixed-point case}

In the last two sections we will deal with reducible polar actions in positive curvature.

The key result in this section is a characterization of Hopf fibrations in our context, that will also play an essential role in the next section. As a corollary we obtain a classification when fixed points are present. We need the following lemma.

Lemma 6.1. (Extension) Let $\left(\mathbb{S}^{n}, \mathrm{G}\right)$ be a fixed-point-free effective polar representation with associated chamber system (building) $\mathscr{C}\left(\mathbb{S}^{n} ; \mathrm{G}\right)$. If $\widetilde{\mathrm{G}} \supset \mathrm{G}$ is a compact connected subgroup of $\operatorname{Aut}_{\text {top }}\left(\mathscr{C}\left(\mathbb{S}^{n}, \mathrm{G}\right)\right)$, then $\widetilde{\mathrm{G}}$ is a Lie group acting linearly on $\mathbb{S}^{n}$.

Proof. We first note that the induced action by $\widetilde{G}$ on $\mathbb{S}^{n}$ is continuous and orbitequivalent to the G-action.

We begin by considering irreducible $G$ representations. In the special case where $G$ acts transitively on $\mathbb{S}^{n}$, a chamber of $\mathscr{C}(M ; \mathrm{G})$ is just a point in $\mathbb{S}^{n}$ and $\operatorname{Aut}_{\text {top }}\left(\mathscr{C}\left(\mathbb{S}^{n}, \mathrm{G}\right)\right)$ is the homeomorphism group of $\mathbb{S}^{n}$ with the compact open topology. Since all $\widetilde{G}$-orbits (there is only one) are locally connected and $\mathbb{S}^{n}$ is a manifold, by [MZ, p. 244, Theorem 1] we have that $\widetilde{G}$ is a Lie group. In fact, by $[\mathrm{Po}], \widetilde{G}$ is a subgroup of $\mathrm{SO}(n+1)$.

In the case where $G$ acts by cohomogeneity 1 or higher on $\mathbb{S}^{n}$, it follows from [Dad] that the action is orbit-equivalent to the isotropy representation of a symmetric space $\mathrm{U} / \mathrm{K}$ of non-compact type. Moreover, $\mathscr{C}\left(\mathbb{S}^{n} ; \mathrm{G}\right)$ is the building at infinity of $\mathrm{U} / \mathrm{K}$ whose (topological) automorphism group is $U$, a Lie group. Thus, $\widetilde{G}$ is a compact subgroup acting isometrically on $\mathrm{U} / \mathrm{K}$, and hence with a fixed point, where the action is linear and orbit-equivalent to the $\mathrm{G}$-action.

In general, the G-action splits into a sum of irreducible subactions. From the above considerations, we conclude that the restriction of $\widetilde{G}$ to each subspace sphere is linear. 
Moreover, since $\widetilde{\mathrm{G}} \subset \mathrm{Aut}_{\mathrm{top}}\left(\mathscr{C}\left(\mathbb{S}^{n}, \mathrm{G}\right)\right)$, it takes chambers to chambers, and hence maps any minimal geodesic between $\mathrm{G}$-invariant subspace spheres to a minimal geodesic between the same invariant spheres. Thus, $\widetilde{G}$ acts linearly (in fact, isometrically) on $\mathbb{S}^{n}$.

We are now ready to prove the following lemma.

Lemma 6.2. (Hopf fibration) Let $\left(\mathbb{S}^{n}, \mathrm{G}\right)$ be a fixed-point-free linear polar action, and $(B, \mathrm{G})$ be a simply connected closed polar manifold. Suppose that $p: \mathbb{S}^{n} \rightarrow B$ is a smooth, G-equivariant, chamber-preserving map with the following property: For each $v \in \mathbb{S}^{n}$, the differential $p_{*}$ on the normal slice at $v$ is a $\mathrm{G}_{v}$-equivariant isomorphism onto the normal slice at $p(v)$, orbit-equivalent to the slice representation of $\mathrm{G}_{p(v)} \supset \mathrm{G}_{v}$. Then $p$ is either a diffeomorphism or a Hopf fibration, up to equivariant diffeomorphism of $B$ (in particular the fibers are great spheres). Moreover, if $\operatorname{dim} B<n$ and $B$ is a sphere, the cohomogeneity is at most 1.

Proof. Note that, by assumption, the chambers $C$ in $\mathbb{S}^{n}$ and $B$ are spherical $k$ simplices, where $k \geqslant 0$ is the cohomogeneity of the actions, and $p$ is surjective. Moreover, $p$ is a submersion, since the differential $p_{*}$ on the tangent space to an orbit is surjective, and by the assumption about slice representations $p_{*}$ is an isomorphism on the normal space to the orbit. Furthermore $p$, when restricted to a section $\Sigma$ in $\mathbb{S}^{n}$, is a cover of a section in $B$. This, in particular, proves our claim when $\operatorname{dim} B=n$.

When $\operatorname{dim} B<n$, we know from $[\mathrm{Br}]$ that the fiber of the submersion $p$ is homeomorphic to $\mathbb{S}^{i}, i \in\{1,3,7\}$, where $i=7$ can only happen when $n=15$. Moreover, from the Gysin exact sequence applied to the fibration $p: \mathbb{S}^{i} \rightarrow \mathbb{S}^{n} \rightarrow B$, it follows that $B$ has the integral cohomology ring of a projective space $\mathbb{F P}^{m}$, with $m \geqslant 1$ and $\mathbb{F}=\mathbb{C}$ or $\mathbb{F}=\mathbb{H}$, if $i \in\{1,3\}$, and $B$ is a homotopy 8 -sphere if $i=7$.

Our proof for the case $\operatorname{dim} B<n$ is anchored at irreducible polar G-representations and polar representations of cohomogeneity at most 1 (on $\mathbb{S}^{n}$ ), in conjunction with the above Lemma 6.1 and the compact transformation group theorem (Theorem 4.9).

- Cohomogeneity $k=0$.

From the list of $\mathrm{G}$ acting transitively and isometrically on $\mathrm{S}^{n}=\mathrm{G} / \mathrm{H}$, it follows directly (and is well known) that $\mathbb{S}^{n}=\mathrm{G} / \mathrm{H} \rightarrow \mathrm{G} / \mathrm{K}$, with fiber $\mathrm{K} / \mathrm{H}=\mathbb{S}^{i}, i \in\{1,3,7\}$, is a Hopf fibration (cf., e.g., [GWZ, Table C]).

- Cohomogeneity $k=1$.

Recall that a cohomogeneity-1 manifold $(M, \mathrm{G})$ is completely determined by its data, i.e., $\mathrm{G}$ and its isotropy groups along a chamber $C$ in $M$. Indeed, if $\mathrm{K}_{ \pm}$are the isotropy groups at the end points $u_{ \pm}$of $C$, and $\mathrm{H}$ is the principal isotropy group along the interior of $C$, then $\mathrm{K}_{ \pm} / \mathrm{H}=\mathbb{S}^{\ell \pm}$ are canonically identified with the normal spheres to the orbits 
at the end points of $C$, and, via the slice theorem and canonical gluing, we have

$$
M=\mathrm{G} \times \mathrm{K}_{-} \mathbb{D}^{\ell-} \cup_{\mathrm{G} / \mathrm{H}} \mathrm{G} \times \mathrm{K}_{+} \mathbb{D}^{\ell_{+}} .
$$

In our case, let $M=\mathbb{S}^{n}$, with data denoted as above. If $\mathrm{K}_{ \pm}^{\prime}$ and $\mathrm{H}^{\prime}$ are the (local) data for $B$ along the chamber $C^{\prime}=p(C)$ in $B$ with end points $u_{ \pm}^{\prime}$, then

$$
B=\mathrm{G} \times \mathrm{K}_{-}^{\prime} \mathbb{D}^{\ell-} \cup_{\mathrm{G} / \mathrm{H}^{\prime}} \mathrm{G} \times \mathrm{K}_{+}^{\prime} \mathbb{D}^{\ell_{+}},
$$

where we have used our assumption $\mathrm{K}_{ \pm}^{\prime} / \mathrm{H}^{\prime}=\mathbb{S}^{\ell_{ \pm}}$, and moreover $\mathrm{K}_{ \pm}^{\prime} / \mathrm{K}_{ \pm}=\mathrm{H}^{\prime} / \mathrm{H}=\mathbb{S}^{i}$ are the fibers of $p$ along $C^{\prime}$. It is important that $p$ is determined by these data as well. We will refer to the dimensions $\left(\ell_{-}, \ell_{+}\right)$of the normal spheres of the singular orbits as the multiplicities of the action.

- Fiber dimensions $i \in\{1,3\}$.

We point out that $B$ is already known, up to equivariant diffeomorphism. Indeed, note that in the classification of cohomogeneity- 1 actions on manifolds with the rational cohomology ring of $\mathbb{F P}^{m}$ due to $[\mathrm{I}]$ and $[\mathrm{Uch}], \mathrm{G}_{2} / \mathrm{SO}(4)$ and the quadrics

$$
\mathrm{SO}(2 m+1) /(\mathrm{SO}(2) \times \mathrm{SO}(2 m-1))
$$

are excluded in our case, since they do not have the correct integral cohomology ring. Thus, from $[\mathrm{I}]$ and $[\mathrm{Uch}]$ we conclude that the $\mathrm{G}$-action on $B$ modulo $\mathrm{K}_{0}$, the identity component of its kernel, is equivariantly diffeomorphic to a linear action on $\mathbb{F P}^{m}$, i.e., there is an equivariant diffeomorphism $f:(B ; \overline{\mathrm{G}}) \rightarrow\left(\mathbb{F P}^{m} ; \overline{\mathrm{G}}\right)$, where the latter action is $\mathbb{F}$-linear, $\bar{G}$ is the connected normal subgroup of $G$ such that $G=K_{0} \cdot \bar{G}$, a product up to finite central quotient.

Consider first the case where the G-representation is irreducible. A classification of these (including their data (corrected in [FGT])) is contained in [GWZ, Table E]. A corresponding classification of those induced on $\mathbb{F P}^{m}$ is contained in [GWZ, Table F].

When $i=3$, i.e., $B \cong \mathbb{H} \mathbb{P}^{m}$, such actions have multiplicity pairs $(1,1)$ and $(2,2 l+1)$, where $m=1$ and $m=l+1, l \geqslant 1$, respectively. In the first case there is only one such action, while in the second one there are two orbit-equivalent actions. From the list of possible G-actions on $\mathbb{S}^{n}$ with these multiplicity pairs, it necessary follows that $\mathrm{K}_{0}=\mathrm{S}^{3}$ acts freely in a linear fashion along the fibers of $f \circ p$ as a subaction of $\mathrm{G}$, and we are done. The same argument works when $i=1$ and the multiplicity pair is $(1, l)$, including an "exceptional case" for each of $(1,5)$ and $(1,6)$. In the remaining cases corresponding to the multiplicity pairs $(2,2 l+1),(4,5)$ and $(9,6)$, either $\mathrm{K}_{0}=\mathrm{S}^{1}$ and we are done, or $\mathrm{K}_{0}$ is trivial. In the latter case, it follows that $\mathrm{G}$ acts almost effectively on $B \cong \mathbb{C P}^{m}$. This, on the other hand, determines all data, and hence $f \circ p: \mathbb{S}^{2 m+1} \rightarrow \mathbb{C P}^{m}$ is the Hopf map, as claimed. 
Now assume that the G-representation is reducible with singular orbits $\mathbb{S}_{ \pm}$and that $\mathbb{S}^{n}=\mathbb{S}_{-} * \mathbb{S}_{+}$. From the homogeneous case it follows that the fibers of $p$ restricted to $\mathbb{S}_{ \pm}$are the fibers of a Hopf fibration. We will show that any fiber of $p$ is a fiber of the uniquely determined Hopf fibration of $\mathbb{S}_{-} * \mathbb{S}_{+}$restricting to the given ones on $\mathbb{S}_{ \pm}$. For any regular point $u^{\prime} \in B$, let $C^{\prime}$ be the unique chamber containing $u^{\prime}$, and let $\mathrm{K}_{ \pm}^{\prime}$ be the isotropy groups at the end points $u_{ \pm}^{\prime}$ of $C^{\prime}$. Also let $C$ be a chamber in $\mathbb{S}^{n}$ with $p(C)=C^{\prime}$ having isotropy groups $\mathrm{K}_{ \pm}$at its end points $u_{ \pm} \in \mathbb{S}_{ \pm}$, and set $\mathrm{K}^{\prime}:=\mathrm{K}_{-}^{\prime} \cap \mathrm{K}_{+}^{\prime}$. From our assumption about slice representations and the natural identifications of the normal spheres to the singular orbits at say $u_{+}^{\prime}$ and $u_{+}$with $\mathbb{S}_{-}$(and vice verse with + and - swapped), it follows that the exponential map from $\mathbb{S}_{u_{+}^{\prime}}^{\perp}$ to $p\left(\mathbb{S}_{-}\right)=B_{-}$is a submersion with fibers identified with the fibers of $p_{-} \mathbb{S}_{-}$. As the same is true with the roles of + and - switched, it is not hard to see that $\mathrm{K}^{\prime}\left(C^{\prime}\right) \cong \mathbb{S}^{i+1}$ is a cohomogeneity- $1 \mathrm{~K}^{\prime}$-submanifold of $B$ with a suspension action, and $p^{-1}\left(\mathrm{~K}^{\prime}\left(C^{\prime}\right)\right)=\mathrm{K}^{\prime}(C) \cong \mathbb{S}^{i} * \mathbb{S}^{i}$ is a cohomogeneity-1 $\mathrm{K}^{\prime}$-submanifold of $\mathbb{S}^{n}$, where the two $\mathbb{S}^{i}$ in the join decomposition are the two Hopf fibers $p^{-1}\left(u_{ \pm}^{\prime}\right)$. Therefore, it suffices to establish our claim when $p: \mathbb{S}^{2 i+1}=\mathbb{S}^{i} * \mathbb{S}^{i} \rightarrow B \cong \mathbb{S}^{i+1}$ and the action on $B$ is a suspension action.

When $i=1$, modulo kernel, the $\mathrm{K}^{\prime}$-action reduces to the reducible $\mathrm{T}^{2}$-action on $\mathbb{S}^{3}$, and the suspension action on the base $\mathbb{S}^{2}$. Therefore, the kernel of $T^{2}$ on the base is $\mathrm{S}^{1} \subset \mathrm{T}^{2}$, acting freely and linearly along the fibers of $p$, and we are done.

When $i=3$, and if the kernel of the action on the base $\mathbb{S}^{4}$ acts transitively on the fibers of $p$, the desired result follows as in the previous case. If not, one checks easily that, modulo kernel, the $\mathrm{K}^{\prime}$-action contains the sum action of $\mathrm{S}^{3} \times \mathrm{S}^{3}$ on $\mathbb{S}^{7}$ as a subaction commuting with a Hopf action given by an $\mathbb{H}$-structure. This determines all data and $p$ is a Hopf map, having as fibers the orbits of the diagonal subgroup $\Delta\left(\mathrm{S}^{3}\right) \subset \mathrm{S}^{3} \times \mathrm{S}^{3}$, the principal isotropy group of the suspension action on the base $\mathbb{S}^{4}$.

- Fiber dimension $i=7$.

In analogy with our applications of [Uch] and [I], we begin by analyzing the action on $B$, where $p: \mathbb{S}^{15} \rightarrow B$ is an equivariant fibration with fiber diffeomorphic to $\mathbb{S}^{7}$.

We claim that the $\mathrm{G}$ action (modulo kernel) on the homotopy 8-sphere $B$ is equivariantly diffeomorphic to the spherical join action by $\mathrm{SO}(2) \mathrm{SO}(7)$, or by $\mathrm{SO}(3) \mathrm{SO}(6)$, or to the suspension action by $\mathrm{SO}(8)$ on $\mathbb{S}^{8}$. Note that, almost effectively, these are also actions by $\operatorname{Spin}(2) \operatorname{Spin}(7), \operatorname{Spin}(3) \operatorname{Spin}(6)=\operatorname{SU}(2)$ SU(4) and Spin(8), all subgroups of Spin(9).

To see this, note that from [GWZ, Table E], the multiplicity pairs in $B$, coinciding with those in $\mathbb{S}^{15}$, are $(4,3)$ (typo in [GWZ]), $(2,5),(1,6)$, and $(1,7)$, for the potential irreducible representations by $\mathrm{Sp}(2) \mathrm{Sp}(2), \mathrm{SU}(2) \mathrm{SU}(4)$ ( or U(2) SU(4)), SO(2) SO(8) and $\mathrm{SO}(2) \operatorname{Spin}(7)$, respectively. In addition, if the G-representation is reducible, the action on $B$ is necessarily a suspension action, and so the multiplicity pair is $(7,7)$. It follows 


\begin{tabular}{|c||c|c|c|c|c|}
\hline $\mathrm{G}$ & Representation & $\mathrm{K}_{-}$ & $\mathrm{K}_{+}$ & $\mathrm{H}$ & $\left(\ell_{-}, \ell_{+}\right)$ \\
\hline \hline $\mathrm{SO}(2) \mathrm{Spin}(7)$ & $\mathbb{R}^{2} \otimes \mathbb{R}^{8}$ & $\Delta(\mathrm{SO}(2)) \mathrm{SU}(3)$ & $\mathrm{G}_{2}$ & $\mathrm{SU}(3)$ & $(1,6)$ \\
\hline $\mathrm{SO}(2) \mathrm{Spin}(7)$ & $\mathbb{R}^{2} \oplus \mathbb{R}^{7}$ & $\mathrm{SO}(2) \mathrm{SU}(4)$ & $\mathrm{Spin}(7)$ & $\mathrm{SU}(4)=\mathrm{Spin}(6)$ & $(1,6)$ \\
\hline $\mathrm{SU}(2) \mathrm{SU}(4)$ & $\mathbb{C}^{2} \otimes \mathbb{C}^{4}$ & $\Delta(\mathrm{SU}(2)) \mathrm{SU}(2)$ & $\mathrm{S}^{1} \cdot \mathrm{SU}(3)$ & $\mathrm{S}^{1} \cdot \mathrm{SU}(2)$ & $(2,5)$ \\
\hline $\mathrm{SU}(2) \mathrm{SU}(4)$ & $\mathbb{R}^{3} \oplus \mathbb{R}^{6}$ & $\mathrm{Sp}(1) \mathrm{Sp}(2)$ & $\mathrm{S}^{1} \mathrm{SU}(4)=\mathrm{S}^{1} \mathrm{Spin}(6)$ & $\mathrm{S}^{1} \cdot \mathrm{Sp}(2)=\mathrm{S}^{1} \mathrm{Spin}(5)$ & $(2,5)$ \\
\hline $\mathrm{S} \sin (8)$ & $\mathbb{R}^{8} \oplus \mathbb{R}^{8}$ & $\mathrm{Spin}(7)$ & $\mathrm{Spin}(7)$ & $\mathrm{G}_{2}$ & $(7,7)$ \\
\hline $\mathrm{S} \sin (8)$ & $\mathbb{R}^{8} \oplus \mathbb{R}^{1}$ & $\mathrm{Spin}(8)$ & $\mathrm{Spin}(8)$ & $\mathrm{Spin}(7)$ & $(7,7)$ \\
\hline
\end{tabular}

Table 1. Fixed point isotropy representations of polar actions on $\mathbb{C a} \mathbb{P}^{2}$.

that the most singular orbit in $B$ has dimension $0,1,2$ or 3 . Thus it is either a point, a circle or a sphere (in the latter cases, since by transversality it is simply connected). Therefore, the dual singular orbit is also a homotopy sphere (or a point) since it has the homotopy type of the complement of the orbit of codimension at least 3, again, e.g., using transversality. Because the singular orbits in $B$ are $\mathrm{G}$-homogeneous spaces, for dimension reasons it follows that $\mathrm{G}$ can neither be $\mathrm{Sp}(2) \mathrm{Sp}(2)$, nor $\mathrm{SO}(2) \mathrm{SO}(8)$ (the former cannot act non-trivially on $\mathbb{S}^{3}$, the latter cannot act transitively on $\left.\mathbb{S}^{6}\right)$. Therefore, the singular orbits of the G-actions on $B$ are, respectively, $\left(\mathbb{S}^{2}, \mathbb{S}^{5}\right),\left(\mathbb{S}^{1}, \mathbb{S}^{6}\right)$ or two points $\left(p_{-}, p_{+}\right)$ corresponding to a representation of SU(2) SU(4) (or its extension), SO(2) Spin(7) or Spin(8) on $\mathbb{S}^{15}$. From isotropy groups data, it follows that the only possible way in which these groups can act by cohomogeneity 1 on $B$, and in particular transitively on the respective pairs of singular orbits, is by the sum action of $\mathrm{SO}(3) \mathrm{SO}(6)$ and $\mathrm{SO}(2) \mathrm{SO}(7)$, and by the suspension action of $\mathrm{SO}(8)$, respectively.

Next, we want to prove that the G-representation on $\mathbb{S}^{15}$ must be one of the tensor representations of SO(2) Spin(7), SU(2) SU(4) or the reducible Spin(8) representation on $\mathbb{S}^{15}$. It remains to exclude the tensor representation of $\mathrm{U}(2) \mathrm{SU}(4)$. To do this, note that if the $\mathrm{U}(2) \mathrm{SU}(4)$ representation descends via $p$ to $B$, then its center $\mathrm{S}^{1}$ must be in the kernel $\mathrm{K}_{0}$ of the $\mathrm{G}$-action on $B$ acting freely along the fibers of $p$. It follows that $p$ induces a fibration $\mathbb{C P}^{7} \rightarrow B=\mathbb{S}^{8}$ with fiber $\mathbb{S}^{7} / \mathrm{S}^{1} \cong \mathbb{C P}^{3}$. But such a fibration does not exist according to $[\mathrm{Ucc}]$.

A set of compatible homomorphisms from the diagrams for the isotropy groups of the tensor representations $\mathrm{SO}(2) \operatorname{Spin}(7), \mathrm{SU}(2) \mathrm{SU}(4)$ and $\operatorname{Spin}(8)$ on $\mathbb{S}^{15}$ to the reducible polar actions on $B$ is exhibited in Table 1 above (cf. [FGT] for a correction of the isotropy groups data for the $\mathrm{SO}(2) \mathrm{Spin}(7)$ case in [GWZ]).

On the other hand, since $\operatorname{Spin}(3) \operatorname{Spin}(6), \mathrm{SO}(2) \operatorname{Spin}(7)$ and $\operatorname{Spin}(8)$ are subgroups of $\operatorname{Spin}(9)$, it follows that they do act on the Cayley plane $F_{4} / \operatorname{Spin}(9)$ in a polar fashion 
with isolated fixed points (cf. [PTh]), and hence act by isometries on the Hopf fibration $\mathbb{S}^{15} \rightarrow \mathbb{S}^{8}$. It is straigtforward to see that, for each of the G-representations, the set of compatible homomorphisms is unique up to conjugation. This proves the desired result.

- Cohomogeneity $k \geqslant 2$.

Whether or not the $\mathrm{G}$ action is irreducible, note that $p$ induces a $\mathrm{G}$-equivariant surjective map between the chamber systems $\mathscr{C}\left(\mathbb{S}^{n}, \mathrm{G}\right) \rightarrow \mathscr{C}(B, \mathrm{G})$ of the same type M. Since $\mathscr{C}\left(\mathbb{S}^{n}, G\right)$ is a building, it is both connected and simply connected. In particular, $\mathscr{C}(B, \mathrm{G})$ is connected.

By our assumption on the slice representations, it follows that $p$ yields an isomorphism between all proper residues of $\mathscr{C}\left(\mathbb{S}^{n}, \mathrm{G}\right)$ and $\mathscr{C}(B, \mathrm{G})$. In particular, we have that $p: \mathscr{C}\left(\mathbb{S}^{n}, \mathrm{G}\right) \rightarrow \mathscr{C}(B, \mathrm{G})$ is a covering map between chamber systems of type $\mathrm{M}$, and hence $\mathscr{C}\left(\mathbb{S}^{n}, \mathrm{G}\right)$ is the universal cover of $\mathscr{C}(B, \mathrm{G})$.

By construction of the chamber topology of the universal cover $\tilde{\mathscr{C}}(B, \mathrm{G})=\mathscr{C}\left(\mathbb{S}^{n}, \mathrm{G}\right)$ in the previous section, it is evident that it coincides with the topology on $\mathscr{C}\left(\mathbb{S}^{n}, \mathrm{G}\right)$ defined using the Hausdorff metric on all compact subsets of $\mathbb{S}^{n}$. The corresponding thick topologies on $\mathscr{C}(B, \mathrm{G})$ and $\mathscr{C}\left(\mathbb{S}^{n}, \mathrm{G}\right)$ yield the original topologies on $B$ and $\mathbb{S}^{n}$, respectively. Moreover, with this topology, $\tilde{\mathscr{C}}(B, \mathrm{G})=\mathscr{C}\left(\mathbb{S}^{n}, \mathrm{G}\right)$ is a compact spherical building.

By Theorem 4.9, we also know that the fundamental group $\pi$ of the cover $\mathscr{C}\left(\mathbb{S}^{n}, \mathrm{G}\right) \rightarrow$ $\mathscr{C}(B, \mathrm{G})$ is a compact subgroup of the topological automorphism group $\operatorname{Aut}_{\text {top }}\left(\mathscr{C}\left(\mathbb{S}^{n}, \mathrm{G}\right)\right)$, and that there is an action by $\widetilde{G} \subset A_{\text {top }}\left(\mathscr{C}\left(\mathbb{S}^{n}, G\right)\right)$ covering the $\bar{G}$-action on $\mathscr{C}$, where $\overline{\mathrm{G}}$ is $\mathrm{G}$ modulo its kernel on $B$, and $\widetilde{\mathrm{G}}$ is an extension of $\overline{\mathrm{G}}$ by $\pi$. Moreover, the actions by $\mathrm{G} \subset \widetilde{\mathrm{G}}$ on $\mathbb{S}^{n}$ are orbit-equivalent, and $B$ is homeomorphic to $\mathbb{S}^{n} / \pi$.

Although in complete generality we do not know much about the automorphism group $\operatorname{Aut}_{\text {top }}\left(\mathscr{C}\left(\mathbb{S}^{n}, \mathrm{G}\right)\right)$, we claim that in our case $\pi \subset \widetilde{G}$ is either $S^{1}$ or $S^{3}$ acting freely on $\mathbb{S}^{n}$ by the Hopf action.

Indeed, when the Coxeter diagram for $\mathrm{M}$ has no isolated nodes, $\operatorname{Aut}_{\text {top }}\left(\mathscr{C}\left(\mathbb{S}^{n}, \mathrm{G}\right)\right)$ is a Lie group by [GKMW] (the rank being at least 3). Moreover, since its maximal compact subgroup acts linearly on $\mathbb{S}^{n}$, the compact group $\pi$ acts linearly and freely on $\mathbb{S}^{n}$, and hence $\pi$ is either trivial, $S^{1}$ or $S^{3}$, acting on $\mathbb{S}^{n}$ by the Hopf action. Notice that $\mathrm{G}$ is either $\overline{\mathrm{G}}$ or $\widetilde{G}$, up to finite kernel.

In general, note that each connected component of the diagram for $\mathrm{M}$ corresponds to a G-invariant linear subsphere, $\mathbb{S}_{i}$ of $\mathbb{S}^{n}$ on which G (modulo its kernel) acts irreducibly in a polar fashion. Moreover, for each $i, \mathscr{C}\left(\mathbb{S}_{i}, \mathrm{G}\right)$ is a compact topological subbuilding of $\mathscr{C}\left(\mathbb{S}^{n}, \mathbf{G}\right)$ invariant under $\widetilde{G}$ covering the chamber subsystem $\mathscr{C}\left(p\left(\mathbb{S}_{i}\right), \mathbf{G}\right)$. Applying Lemma 6.1 , we conclude that the compact group $\widetilde{G}$ is a Lie group that acts isometrically and is orbit-equivalent to the action by $G$ on $\mathbb{S}^{n}$. Thus also $\pi$ is a compact Lie group 
acting isometrically on $\mathbb{S}^{n}$, and the fibration $p: \mathbb{S}^{n} \rightarrow B$ is the orbit map by the free action of $\pi$.

We are now ready to prove the following theorem.

THEOREM 6.4. Let $M$ be a simply connected, compact, positively curved polar Gmanifold. If $M^{\mathrm{G}} \neq \varnothing$ then $(M, \mathrm{G})$ is equivariantly diffeomorphic to an isometric, polar action of $\mathrm{G}$ on a compact rank-1 symmetric space.

Proof. We will see in particular that $M$ is a sphere if and only if the section $\Sigma$ is a sphere.

Let us first deal with the case where

- $\Sigma$ is a $k$-sphere, $k \geqslant 2$.

Recall, by Proposition 3.6, that in this case $M^{\mathrm{G}}=\Sigma^{\mathrm{W}}=: \mathbb{S} \subset \Sigma=\mathbb{S} * \mathbb{S}^{\ell}$, and $C=M / \mathrm{G}=$ $\Sigma / \mathrm{W}=\mathbb{S} * \Delta$, where $\Delta=\mathbb{S}^{\ell} / \mathrm{W}$ and $\operatorname{dim} \Delta=\ell \geqslant 1$.

The smooth spherical join description $\Sigma=\mathbb{S} * \mathbb{S}^{\ell}$ yields a decomposition of $\Sigma$ as a union of tubular neighborhoods of $\mathbb{S}$ and of $\mathbb{S}^{\ell}$. Applying $G$ gives a smooth decomposition of $M$ into a union of tubular neighborhoods of $\mathbb{S}=M^{\mathrm{G}}$ and the $\mathrm{G}$-invariant manifold $G \mathbb{S}^{\ell}=: \mathbb{S}^{\prime} \subset M$. (In the metric chosen, note that the cut locus of $\mathbb{S}$ in $M$ is $\mathbb{S}^{\prime}$ and vice versa, at distance $\frac{1}{2} \pi$ from one another.) Note that $\mathbb{S}^{\prime}$ is a polar G-manifold with section $\mathbb{S}^{\ell}$, polar group $W$ and $\mathbb{S}^{\prime} / G=\mathbb{S}^{\ell} / \mathrm{W}=\Delta$. Moreover, if $\mathbb{S}^{0}=\left\{p_{-} \cup p_{+}\right\} \subset \mathbb{S}$ is a pair of antipodal points in $\mathbb{S}$, we see that $\mathrm{G} \mathbb{S}^{\ell+1}=\mathrm{G}\left(\left\{p_{-} \cup p_{+}\right\} * \mathbb{S}^{\ell}\right)$ is a $\mathrm{G}$-invariant polar submanifold $N \subset M$ with two isolated fixed points $p_{ \pm}$, section $\Sigma_{0}:=\left\{p_{-} \cup p_{+}\right\} * \mathbb{S}^{\ell} \subset \Sigma$ and polar group W. From this, it in particular follows that $\mathbb{S}^{\prime}$ is equivariantly diffeomorphic to the unit sphere $\mathbb{S}^{\perp}$ at a fixed point, say $p_{-}$, of G in $N$, and that $N$ is equivariantly diffeomorphic to the suspension of this. Of course $\mathbb{S}^{\perp}$ is the normal sphere of $\mathbb{S}=M^{\mathrm{G}}$ in $M$ at $p_{-}$, and a similar argument now shows that $M$ is equivariantly diffeomorphic to $\mathbb{S} * \mathbb{S}^{\perp}$, where $G$ acts trivially on $\mathbb{S}$ and, by the isotropy representation of $G$, on the normal sphere $\mathbb{S}^{\perp}$.

We now turn to the case where

- $\Sigma$ is a projective $k$-space, $k \geqslant 2$.

Since $\Sigma^{*}=\Sigma / \mathrm{W}$ is a spherical $k$-simplex $\Delta$ and $M^{\mathrm{G}} \subset \Sigma^{\mathrm{W}}$, we know that $M^{\mathrm{G}}$ is contained in the vertices of any chamber $C=\Delta=M / \mathrm{G}=\Sigma / \mathrm{W}$ by Proposition 2.8.

Let $p_{0} \in M^{\mathrm{G}}$ be such an isolated fixed point, and $\Delta_{0}$ be the chamber face opposite $p_{0}$ in a fixed chamber $C=\Delta$, i.e., $C=\Delta=p_{0} * \Delta_{0}$. It follows that $B:=\mathrm{G} \Delta_{0} \subset M$ is a polar space form G-submanifold of $M$ with section $\mathbb{R P}^{k-1} \subset \Sigma=\mathbb{R} \mathbb{P}^{k}$ and polar group induced from W. Arguing as above, $M$ is the union of a ball centered at $p_{0}$ and a tubular neighborhood of $B$. (In the chosen metric, $B$ is the cut locus of $p_{0}$ and vice versa at distance $\frac{1}{2} \pi$ ). In particular, we have an equivariant sphere fiber bundle $p: \mathbb{S} \rightarrow B$ (with non-trivial fiber) between polar G-manifolds with the same orbit space $\Delta_{0}$, where $\mathbb{S}$ is 
the unit sphere at $p_{0}$. Note also that, for any $0<r<\frac{1}{2} \pi$, the metric $r$-sphere $\mathbb{S}\left(p_{0}, r\right)$ centered at $p_{0}$ is a polar $\mathbf{G}$-manifold equivariantly diffeomorphic to $\mathbb{S}$ via scaling and $\exp _{p_{0}}$. Moreover, $\mathbb{S}\left(p_{0}, r\right)$ coincides with the boundary $\mathbb{S}\left(B ; \frac{1}{2} \pi-r\right)$ of the $\left(\frac{1}{2} \pi-r\right)$-tube $\mathbb{D}\left(B ; \frac{1}{2} \pi-r\right)$ of $B$, and in this way $p$ can also be viewed as the projection from the unit normal sphere bundle $\mathbb{S}^{\perp}(B)$ of $B$ to $B$. By transversality, we see that $B$ is simply connected. From this description, it follows that $M$ is equivariantly diffeomorphic to a projective space, once it is established that $p$ is a Hopf fibration. To see this, it remains to check the assumption on the slice representations in Lemma 6.2.

Let $\gamma$ be a geodesic in $C$ from $p_{0}$ to $q \in \Delta_{0}$ which is perpendicular to $\Delta_{0}$. By the slice theorem, $\mathrm{G} \times{ }_{\mathrm{G}_{q}} \widehat{V}_{q}$ is a G-equivariant tubular neighborhood of the orbit $\mathrm{G}(q)$, where $\widehat{V}_{q}$ is the slice in $M$. Since $B$ is $\mathrm{G}$-invariant, we get a $\mathrm{G}_{q}$-invariant decomposition $\widehat{V}_{q}=V_{q} \oplus V_{q}^{\perp}$, where $V_{q}$ is the slice in $B$, and $V_{q}^{\perp}$ is the normal space to $B$ at $q$. Note that, from the slice representation of $\mathrm{G}_{q}$ on $V_{q} \oplus V_{q}^{\perp}$, the slice $\widehat{V}_{x}$ for $\mathrm{G}_{x}$ at $x \in \gamma$ different from $q$ is naturally identified with $V_{q} \oplus T_{x} \gamma$. Therefore, the slice of the orbit at $x$ inside $\mathbb{S}\left(B ; \frac{1}{2} \pi-r\right)$ is canonically identified with $V_{q}$. Moreover, the orbit space $V_{q} / \mathrm{G}_{x}=V_{q} / \mathrm{G}_{q}$ is a cone over the space of directions at $q \in \Delta_{0}$. The desired result follows.

\section{Fixed-point-free reducible actions}

In all remaining cases, the orbit space $M^{*}=\Sigma^{*}$ is a simplex $\Delta$ isometric to all chambers in $M$. Moreover, $\Delta$ is a spherical join $\Delta=\Delta_{-} * \Delta_{+}=\Delta^{m_{-}} * \Delta^{m_{+}}$, corresponding to two dual W-invariant subsections $\Sigma_{-}$and $\Sigma_{+}$, where $\Sigma_{ \pm}$are either $\mathbb{S}^{m_{ \pm}}$or the projective spaces $\mathbb{R} \mathbb{P}^{m_{ \pm}}$

Viewing $\Delta$ also as a subset of a fixed section $\Sigma$, clearly $B_{-}=\mathrm{G} \Delta^{m_{-}}$and $B_{+}=\mathrm{G} \Delta^{m_{+}}$ are two polar G-submanifolds in $M$ with sections $\Sigma_{-}, \Sigma_{+} \subset \Sigma$ and Weyl group W (modulo kernel). In particular, $B_{ \pm}$are polar space forms of spherical type. Moreover, just like $\Delta$ can be viewed as the union of two tubular neighborhoods of the $\Delta^{m_{ \pm}}, M$ is the union of tubular neighborhoods of the G-submanifolds $B_{ \pm}$.

In the remaining cases where no fixed points are present, our first goal is to exhibit a geometric description of $M$ as a projective space in which $B_{ \pm}$is a dual pair of projective subspaces. The pivotal steps are to show that these pairs are the cut loci of one another, and that for each point $p_{ \pm} \in B_{ \pm}$, the exponential map (up to scaling) from the unit normal sphere at $p_{ \pm}$to $B_{ \pm}$defines a map to $B_{\mp}$, which in turn is a Hopf fibration. This is in spirit achieved by reducing it to the fixed-point case, where the groups in question are the isotropy groups at $p_{ \pm}$. Analyzing and making full use of equivariant restrictions forced by this description will then yield a proof of our main result in this section. 
TheOREM 7.1. (Non-fixed point) A reducible fixed-point-free polar action on a simply connected, positively curved manifold is equivariantly diffeomorphic to an isometric polar action on a rank-1 symmetric space, excluding the Cayley plane.

Note that, when $m_{+} \geqslant 1$, say, the slice representation at each vertex of $\Delta_{+} \subset \Delta$ is reducible. In particular, all vertex representations are reducible except possibly the one corresponding to $\Delta_{-}$, say, when it is a point.

The following is a key step based on the primitivity lemma (Lemma 3.2).

Lemma 7.2. (Dual generation) For any regular pair $p_{ \pm} \in B_{ \pm}$, the action of the isotropy groups $\mathrm{G}_{p_{ \pm}}$restricted to $B_{\mp}$ is orbit-equivalent to the action of $\mathrm{G}$ restricted to $B_{\mp}$.

Proof. By the primitivity theorem, $\mathrm{G}_{p_{-}}$is generated by the face isotropy groups $\mathrm{G}_{v_{1}}, \ldots, \mathrm{G}_{v_{m_{+}+1}}$ of the faces $\Delta_{-} * \Delta_{v_{i}}^{m_{+}-1}$ containing $\Delta_{-}$, and similarly $\mathrm{G}_{p_{+}}$is generated by the remaining face isotropy groups $\mathrm{G}_{u_{1}}, \ldots, \mathrm{G}_{u_{m_{-}+1}}$ namely of the faces $\Delta_{u_{j}}^{m_{-}-1} * \Delta_{+}$ containing $\Delta_{+}$. Note that any face containing $\Delta_{-}$is perpendicular to any face containing $\Delta_{+}$. In particular, if $G_{v, u}$ is the isotropy group at an intersection point of two such faces with isotropy groups $\mathrm{G}_{v}$ and $\mathrm{G}_{u}$, the slice representation of $\mathrm{G}_{v, u}$ restricted to the normal sphere of its fixed-point set is a reducible cohomogeneity- 1 action with singular isotropy groups $\mathrm{G}_{v}$ and $\mathrm{G}_{u}$. As a special case of the primitivity theorem, we already know that $\mathrm{G}_{v}$ and $\mathrm{G}_{u}$ generate $\mathrm{G}_{v, u}$. However, since the action is reducible, we have that actually $\mathrm{G}_{v} \mathrm{G}_{u}=\mathrm{G}_{u} \mathrm{G}_{v}=\mathrm{G}_{v, u}$ as sets. Notice that this is equivalent to the fact that in the slice representation of $\mathrm{G}_{v, u}$, the isotropy group $\mathrm{G}_{v}$ is transitive on the opposite singular orbit and vice versa.

We now claim that $\mathrm{G}=\mathrm{G}_{p_{-}} \mathrm{G}_{p_{+}}$. From the primitivity lemma, we know that any $g \in \mathrm{G}$ can be written as a word of elements from $\mathrm{G}_{v_{1}}, \ldots, \mathrm{G}_{v_{m_{+}+1}}, \mathrm{G}_{u_{1}}, \ldots, \mathrm{G}_{u_{m_{-}+1}}$. Using that $\mathrm{G}_{v_{i}} \mathrm{G}_{u_{j}}=\mathrm{G}_{u_{j}} \mathrm{G}_{v_{i}}$ for all $i=1, \ldots, m_{+}+1$ and $j=1, \ldots, m_{-}+1$, we can rewrite any such word also as a word in the $\mathrm{G}_{v}$ 's times a word in the $\mathrm{G}_{u}$ 's, i.e., $\mathrm{G}=\mathrm{G}_{p_{-}} \mathrm{G}_{p_{+}}$. The same reasoning shows that $\mathrm{G}=\mathrm{G}_{p_{+}} \mathrm{G}_{p_{-}}$, and hence completes the proof of the lemma.

The above lemma will allow us to use the input from the previous section. For this, we let $\Gamma\left(p_{ \pm}\right)$be the set consisting of all minimal geodesics from regular points $p_{ \pm}$to $B_{\mp}$. In addition to viewing this as a set of geodesics, we will also view it as a subset of $M$ whose points are the points of all those geodesics. As such, it can also be described as $\Gamma\left(p_{ \pm}\right)=\mathrm{G}_{p_{ \pm}}\left(p_{ \pm} * \Delta_{\mp}\right) \subset M$. Note that $\Gamma\left(p_{-}\right) \cap \Gamma\left(p_{+}\right)$is the set of all minimal geodesics joining $p_{-}$and $p_{+}$. Since $\mathrm{G}_{p_{ \pm}}$is independent of $p_{ \pm}$, we will use the notation $\mathrm{G}_{ \pm}$instead.

It will also be useful to let $\Gamma\left(p_{ \pm}\right)(r)$ denote the subset of $\Gamma\left(p_{ \pm}\right)$made of all the points at distance $r$ from $p_{ \pm}$, and $\widehat{\Gamma}\left(p_{ \pm}\right)$denote the negative of the terminal directions of the geodesics in $\Gamma\left(p_{ \pm}\right)$. 
Remark 7.3. The following are immediate consequences of the dual generation lemma (Lemma 7.2), and the decomposition of a section $\Sigma \supset \Sigma_{ \pm}$corresponding to $\Delta=\Delta_{-} * \Delta_{+}$.

- The cut locus $C\left(B_{ \pm}\right)=B_{\mp}$, and $B_{ \pm}$are at distance $\frac{1}{2} \pi$ from one another.

- $\Gamma\left(p_{ \pm}\right) \backslash B_{\mp}$ is a smooth submanifold of $M$ diffeomorphic to the open $\frac{1}{2} \pi$-ball in the normal space $T_{ \pm}^{\perp}$ to $B_{ \pm}$at $p_{ \pm}$via the exponential map.

- The map $\gamma_{p \pm}: \mathbb{S}_{p_{ \pm}}^{\perp} \rightarrow B_{\mp}$ taking a unit vector to the corresponding geodesic at time $\frac{1}{2} \pi$ is smooth, $\mathrm{G}_{ \pm}$equivariant and takes chambers to chambers.

Moreover, we have the following lemma.

LEMma 7.4. The map $\gamma_{p \pm}$ is a $\mathrm{G}_{ \pm}$-equivariant Hopf fibration.

Proof. For the sake of simplicity, we will use $\bar{x}$ to denote the image $\gamma_{p \pm}(x)$ of $x \in \mathbb{S}_{p_{ \pm}}^{\perp}$. Since $\gamma_{p \pm}$ is a smooth $\mathrm{G}_{ \pm}$-equivariant map that takes chambers to chambers and with the same orbit space, by the Hopf lemma (Lemma 6.2) it remains to verify that the slice representations of $\mathrm{G}_{ \pm, v}$ and $\mathrm{G}_{ \pm, \bar{v}}$ are orbit equivalent. For this it suffices to show that the dimensions of the corresponding slices agree, or equivalently that the corresponding principal orbits of isotropy groups have the same dimension. So let $x$ be a point of principalorbit type of the slice representation of $\mathrm{G}_{ \pm, v}$. We claim that $\operatorname{dim}\left(\mathrm{G}_{ \pm, v}(x)\right)=\operatorname{dim}\left(\mathrm{G}_{ \pm, \bar{v}}(\bar{x})\right)$. Note that $\mathrm{G}_{ \pm, \bar{v}}=\mathrm{G}_{ \pm, \bar{x}} \mathrm{G}_{ \pm, v}=\mathrm{G}_{ \pm, v} \mathrm{G}_{ \pm, \bar{x}}$, where the latter follows from the dual generation lemma (Lemma 7.2). Therefore, the orbit $\mathrm{G}_{ \pm, \bar{v}}(\bar{x})=\mathrm{G}_{ \pm, \bar{v}} / \mathrm{G}_{ \pm, \bar{x}}=\mathrm{G}_{ \pm, v} \mathrm{G}_{ \pm, \bar{x}} / \mathrm{G}_{ \pm, \bar{x}}$. In particular, $\mathrm{G}_{ \pm, v}$ acts transitively on this orbit with isotropy group $\mathrm{G}_{ \pm, v} \cap \mathrm{G}_{ \pm, \bar{x}}$. However, since clearly $\mathrm{G}_{ \pm, v} \cap \mathrm{G}_{ \pm, \bar{x}}=\mathrm{G}_{ \pm, x}$, this completes the proof.

The following plays a pivitol role in the geometric and equivariant description of $M$.

Lemma 7.5. (Reduction) For all regular $p_{ \pm} \in B_{ \pm}, \Gamma\left(p_{ \pm}\right)$are $\mathrm{G}_{ \pm}$-invariant submanifolds of $M$. Moreover, $\Gamma\left(p_{ \pm}\right)$is $\mathrm{G}_{ \pm}$-equivariantly diffeomorphic to

$$
\begin{cases}\mathbb{D}_{p_{ \pm}}^{\perp}, & \text { if the section is a sphere, } \\ \text { a complex or quaternionic projective space, } & \text { if the section is a projective space. }\end{cases}
$$

Proof. The key issue is to see that $\Gamma\left(p_{ \pm}\right)$are submanifolds, as claimed. From the remark above, this is clear except along $B_{\mp} \subset \Gamma\left(p_{ \pm}\right)$.

From the Hopf lemma and Lemma 7.4 above, we know that $\gamma_{p_{ \pm}}: \mathbb{S}_{p_{ \pm}}^{\perp} \rightarrow B_{\mp}$ is either a diffeomorphism or a Hopf map. Clearly, $\Gamma\left(p_{-}\right) \cap \Gamma\left(p_{+}\right)$is in bijective correspondence with the fiber of $\gamma_{p_{-}}$over $p_{+}$and the fiber of $\gamma_{p_{+}}$over $p_{-}$, when viewing it as the set of minimal geodesics between $p_{-}$and $p_{+}$. In particular, both maps are of the same type, corresponding to $\Gamma\left(p_{-}\right) \cap \Gamma\left(p_{+}\right)$being either one geodesic, an $\mathbb{S}^{1}$-, an $\mathbb{S}^{3}$ - or an $\mathbb{S}^{7}$-family of geodesics. Moreover, this description also shows that the linear span of the initial vectors of the geodesics in $\Gamma\left(p_{-}\right) \cap \Gamma\left(p_{+}\right)$at both $p_{-}$and $p_{+}$are linear subspaces of the corresponding normal spaces to $B_{\mp}$. 
Now consider the initial vectors of the geodesics in $\Gamma\left(p_{-}\right)$starting at $B_{+}$. This subset $\widehat{\Gamma}\left(p_{-}\right)$of the unit normal bundle $T_{1}^{\perp} B_{+}$of $B_{+}$is canonically a smooth submanifold diffeomorphic to $\mathbb{S}_{p_{-}}^{\perp}$ via, say, $\Gamma\left(p_{-}\right)\left(\frac{1}{2} \pi-1\right)$. In particular, it is a smooth section of the unit normal bundle to $B_{+}$when each $\Gamma\left(p_{-}\right) \cap \Gamma\left(p_{+}\right)$is just one geodesic, or equivalently $\gamma_{p_{-}}: \mathbb{S}_{p_{-}}^{\perp} \rightarrow B_{+}$is a diffeomorphism. In the other cases, it is the unit sphere bundle of a smooth linear subbundle of the normal bundle to $B_{+}$and $\gamma_{p_{-}}: \mathbb{S}_{p_{-}}^{\perp} \rightarrow B_{+}$is a Hopf fibration. By equivariance then clearly each $\Gamma\left(p_{\mp}\right)$ is $\mathrm{G}_{\mp}$-equivariantly diffeomorphic to a complex or quaternionic space, or to the Cayley plane if $\Gamma\left(p_{-}\right) \cap \Gamma\left(p_{+}\right)$is an $\mathbb{S}^{7}$ family of geodesics. Since by assumption neither $B_{ \pm}$is a point, the latter case does not appear. Indeed, if so, both $B_{ \pm}$would be homotopy 8-spheres. Moreover, from the geometric decomposition and Poincaré duality, it follows that $M$ would be a 24dimensional manifold having as integral cohomology algebra a truncated polynomial algebra with generator in degree 8. This contradicts a well-known topological theorem (cf. [Ha, p. 498, Corollary 4]).

Having dealt with all cases where the diagram for the Coxeter matrix has no isolated nodes, and where the action has fixed points, we assume from now on that $B_{-}$is an orbit corresponding to an isolated node of the diagram. Thus, we will use a decomposition

$$
\Delta=\Delta_{-} * \Delta_{+},
$$

where $\Delta_{-}=\Delta^{0}=p_{-}$is a vertex corresponding to an isolated node, and $\Delta_{+}$corresponds to the rest of the diagram. In particular, $\mathrm{G}_{+}$acts transitively on $B_{-}$, as well as on each normal sphere $\mathbb{S}_{+}^{\perp}$ to $B_{+}$along $\Delta_{+}$.

We are now ready to complete the proof of Theorem 7.1.

Proof of Theorem 7.1. We first consider the case where the section $\Sigma=\Sigma_{-} * \Sigma_{+}$is a sphere.

By Lemma 7.5 , the $\mathrm{G}_{\mp}$-action on $B_{ \pm}$is equivariantly equivalent to the slice representation on the normal sphere $\mathbb{S}_{p_{\mp}}^{\perp}$, which we will denote by $\mathbb{S}\left(V_{ \pm}\right)=\mathbb{S}_{ \pm}$. Note that $\mathrm{G}$, as well as $\mathrm{G}_{+}$, acts transitively on $B_{-}$, since $\Delta_{-}$is a point. In particular $\mathrm{G}$ acts linearly on $\mathbb{S}_{-}$identified with $B_{-}$. If also the $\mathrm{G}_{\text {-action on }} B_{+}$when identified with $\mathbb{S}_{+}$is linear, we claim that the induced sum action on $\mathbb{S}\left(V_{-} \oplus V_{+}\right)$is equivalently diffeomorphic to the $\mathrm{G}$-action on $M$. To see this, choose $p_{-}^{*} \in \mathbb{S}_{-}$with $\mathrm{G}_{p_{-}^{*}}=\mathrm{G}_{p_{-}}=\mathrm{G}_{-}$and a $\mathrm{G}_{-}$-equivariant diffeomorphism from $\Gamma\left(p_{-}\right)$to the join $p_{-}^{*} * \mathbb{S}_{+} \subset \mathbb{S}_{-} * \mathbb{S}_{+}$. This extends to a well-defined Gequivariant diffeomorphism from $M$ to $\mathbb{S}_{-} * \mathbb{S}_{+}$by invariance. The proof is completed now by Lemma 6.1 , since the polar $\mathrm{G}$-action on $B_{+}$is linear-orbit equivalent to the $\mathrm{G}_{-}$-action.

Now suppose that $\Sigma$ is a projective space. 
By Lemma 7.5, the Cayley plane cannot appear, and moreover $B_{ \pm}$are both projective spaces over $\mathbb{F}$, with $\mathbb{F}=\mathbb{C}$ or $\mathbb{F}=\mathbb{H}$. In fact, for each regular $p_{ \pm} \in B_{ \pm}$, the slice representation of $\mathrm{G}_{ \pm}$restricted to the normal space $V_{ \pm}$at $p_{ \pm}$to $B_{ \pm}$preserves an $\mathbb{F}$-structure and descends to a polar action on $B_{\mp}$ which is orbit-equivalent to the restriction of $G$ to $B_{\mp}$. This will guide us to the construction of a representation of $\mathrm{G}$ (or frequently an extension of it) on $V_{+} \oplus V_{-}$preserving an $\mathbb{F}$-structure, which together with the scalar multiplication of $\mathbb{F}^{*}$ is polar, such that the induced $\mathrm{G}$-action on the projective space $\mathbb{F} \mathbb{P}\left(V_{+} \oplus V_{-}\right)$is equivariantly equivalent to that of $\mathrm{G}$ on $M$.

We divide the proof into two cases corresponding to (a) $\operatorname{dim} B_{-}>2$ and (b) $\operatorname{dim} B_{-}=2$ (noting that $\operatorname{dim} B_{-}<2$ is covered by the fixed-point case).

Throughout, $\mathrm{K}_{ \pm} \triangleleft \mathrm{G}$ will denote the identity component of the kernel of the $\mathrm{G}$-action restricted to $B_{ \pm}$.

In case (a), we make the following claim: There is a normal subgroup $\mathrm{H} \triangleleft \mathrm{K}_{+}$acting transitively on the normal spheres to $B_{+}$such that $\mathrm{K}_{+}=\mathrm{H} \cdot \mathrm{K}_{0}$, with $\mathrm{K}_{0} \in\left\{1, \mathrm{~S}^{1}, \mathrm{~S}^{3}\right\}$, is the identity component of the kernel of the action by $\mathrm{K}_{+}$on $B_{-}$. We will see later on that $\mathrm{K}_{0}=1$ when $\mathbb{F}=\mathbb{H}$, and that $\mathrm{K}_{0} \in\left\{1, \mathrm{~S}^{1}\right\}$ when $\mathbb{F}=\mathbb{C}$.

To prove the claim, note that $\operatorname{dim} \Delta_{+} \geqslant 1$. Choose a pair of vertices $v_{1}, v_{2} \in \Delta_{+}$and consider the slice representations at the vertices. By Lemma 7.7 below, it follows by assumption on $\operatorname{dim} B_{-}$, that up to a finite cover, there is a normal subgroup of rank at least 2 (of simple type), say $\mathrm{H}_{v_{i}} \triangleleft \mathrm{G}_{v_{i}}$ for $i=1,2$, acting transitively on the normal sphere to $B_{+}$at $v_{i}$, but trivially on the slice tangent to $B_{+}$. Clearly each $\mathrm{H}_{v_{i}}$ is also a normal (simple) subgroup in the principal isotropy group $\mathrm{G}_{+}$of the $\mathrm{G}$-action on $B_{+}$. As $G_{+}$(modulo kernel) has a unique normal simple subgroup (of rank at least 2) acting transitively on the normal sphere, it follows that $\mathrm{H}_{v_{1}}=\mathrm{H}_{v_{2}} \triangleleft \mathrm{G}_{+}$, and will be denoted by $\mathrm{H}$. By primitivity, $\mathrm{G}=\left\langle\mathrm{G}_{v_{1}}, \mathrm{G}_{v_{2}}\right\rangle$, and thus $\mathrm{H}$ is a normal subgroup in $\mathrm{G}$. Therefore, $B_{+}$is fixed pointwise by $\mathrm{H}$. In particular, $\mathrm{H} \triangleleft \mathrm{K}_{+}$acts transitively on $B_{-}$with kernel $\mathrm{K}_{0}$, and thus $\mathrm{K}_{+}=\mathrm{H} \cdot \mathrm{K}_{0}$.

Since $\mathrm{K}_{+} \triangleleft \mathrm{G}$, we can write $\mathrm{G}=\mathrm{K}_{+} \cdot \mathrm{L}=\mathrm{H} \cdot \mathrm{K}_{0} \cdot \mathrm{L}$, where $\mathrm{L} \triangleleft \mathrm{G}$ is a connected normal subgroup which clearly acts almost effectively on $B_{+}$in a polar fashion. Since $B_{-}$is a projective space over $\mathbb{C}$ or $\mathbb{H}$ of dimension at least 4 , and therefore any almost effective transitive action on it is the linear action by $\mathrm{SU}(k+1)$ or $\mathrm{Sp}(k)$ for some $k \geqslant 2$ (cf. [O, pp. 264-265]), we conclude that $\mathrm{H}=\mathrm{SU}(k+1)$ or $\mathrm{H}=\mathrm{Sp}(k)$. Thus, for the kernel of the $\mathrm{G}=\mathrm{H} \cdot \mathrm{K}_{0} \cdot \mathrm{L}$ action on $B_{-}$, we get $\mathrm{K}_{-}=\mathrm{K}_{0} \cdot \mathrm{L}$. In particular, $\mathrm{K}_{0}=\mathrm{K}_{-} \cap \mathrm{K}_{+}$fixes both $\mathrm{B}_{ \pm}$ pointwise, and acts almost effectively on the normal spheres to $B_{ \pm}$(when non trivial), preserving all $\Gamma\left(p_{-}\right) \cap \Gamma\left(p_{+}\right)$.

In summary, the "face" isotropy groups $G_{ \pm}$and their kernels $K_{ \pm}$can be read off 
from the following group diagram:

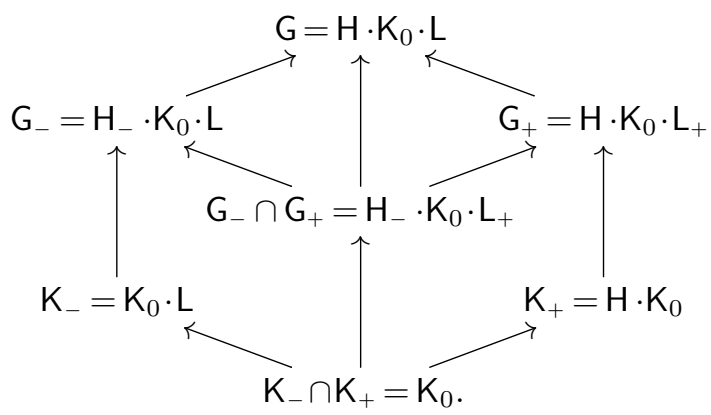

Here $\mathrm{H}_{-}$(resp. $\mathrm{L}_{+}$) is the principal isotropy group of $\mathrm{H}$ on $B_{-}$(resp. of $\mathrm{L}$ on $B_{+}$). Thus, corresponding to the possible $\mathrm{H}$ above, we have $\mathrm{H}_{-}=\mathrm{U}(k), \mathrm{H}=\mathrm{Sp}(k-1) \mathrm{S}^{1}$ or $\mathrm{H}=\mathrm{Sp}(k-1) \mathrm{Sp}(1)$. Let $\mathrm{H}_{0} \triangleleft \mathrm{H}_{-}$denote the normal factor $\mathrm{S}^{1}$ or $\mathrm{Sp}(1)$ of $\mathbf{H}_{-}$. Thus, $\mathrm{H}_{0}=\mathrm{S}^{1}$ corresponds to $\mathbb{F}=\mathbb{C}$ and $\mathrm{H}_{0}=\mathrm{Sp}(1)$ to $\mathbb{F}=\mathbb{H}$. For this we have:

- $\mathrm{H}_{0}$ acts freely on $\mathbb{S}\left(V_{-}\right)$along the Hopf fibers.

To see this, consider for any fixed $p_{+} \in B_{+}$, the Hopf map $\gamma_{p_{+}}: \mathbb{S}_{p_{+}}^{\perp} \rightarrow B_{-}$. From the transitive actions by $\mathrm{H}$ on $\mathbb{S}_{p_{+}}^{\perp}$ descending to $B_{-}$, we know that $\mathrm{H}_{0}$ acts freely along the fibers of $\gamma_{p_{+}}$. Note that these fibers, for $p_{-} \in B_{-}$, are in one-to-one correspondence with $\Gamma\left(p_{-}\right) \cap \Gamma\left(p_{+}\right)$. Turning things around, these are also in one-to-one correspondence with the fibers of the Hopf map $\gamma_{p_{-}}: \mathbb{S}_{p_{-}}^{\perp} \rightarrow B_{+}$, where now $p_{-}$is fixed. This proves the assertion and has the following consequence:

- $\mathrm{K}_{0}$ is trivial when $\mathbb{F}=\mathbb{H}$.

Indeed, since both $\mathrm{K}_{0}$ and $\mathrm{H}_{0}=\mathrm{Sp}(1)$ act (almost) effectively on $\mathbb{S}\left(V_{-}\right)$, this follows from Lemma 7.9 below, because the slice representation of $G_{-}$on $V_{-}$descends to a fixed-point-free action on $B_{+}$, with $\mathrm{H}_{0} \cdot \mathrm{K}_{0} \triangleleft \mathrm{H}_{-} \cdot \mathrm{K}_{0}$ in its kernel.

We now proceed to set up a projective model $\mathbb{F} \mathbb{P}\left(V_{+} \oplus V_{-}\right)$, with a linear polar Gaction with the field $\mathbb{F}=\mathbb{C}$ or $\mathbb{F}=\mathbb{H}$, as indicated in our strategy above.

Consider the product representation on $V_{+} \oplus V_{-}$by $\mathrm{K}_{+} \times \mathrm{K}_{-}=\mathrm{H} \cdot \mathrm{K}_{0} \times \mathrm{K}_{0} \cdot \mathrm{L}$, which on each summand preserves an $\mathbb{F}$-structure, i.e., descends to a polar action on $\mathbb{F} \mathbb{P}\left(V_{+}\right)$and on $\mathbb{F} \mathbb{P}\left(V_{-}\right)$, respectively. When $\mathrm{K}_{0}$ is trivial, obviously the sum $\mathbb{F}$-structure is preserved as well, and the $\mathrm{K}_{+} \times \mathrm{K}_{-}=\mathrm{H} \times \mathrm{L}$ action descends to a polar action on $\mathbb{F} \mathbb{P}\left(V_{+} \oplus V_{-}\right)$. When $\mathrm{K}_{0}=\mathrm{S}^{1}$, and hence $\mathbb{F}=\mathbb{C}$, the action by the diagonal $\mathrm{S}^{1}=\Delta\left(\mathrm{K}_{0}\right) \triangleleft \mathrm{K}_{0} \times \mathrm{K}_{0}$ defines a $\mathbb{C}$ structure on the sum preserved by $\mathrm{K}_{+} \times \mathrm{K}_{-}$descending to a polar action by $\mathrm{G}=\mathrm{H} \cdot \mathrm{K}_{0} \cdot \mathrm{L}$ on $\mathbb{F P}\left(V_{+} \oplus V_{-}\right)$. These are the models.

Given such a model, fix $p_{-} \in B_{-}$, and choose a point $p_{-}^{*} \in \mathbb{F} \mathbb{P}\left(V_{+}\right)$so that $\mathrm{G}_{p_{-}^{*}}=\mathrm{G}_{p_{-}}$. By Lemma $7.5, \Gamma\left(p_{-}\right)$is $\mathrm{G}_{p_{-}}$-equivariantly diffeomorphic to the linear projective subspace of $\mathbb{F P}\left(V_{+} \oplus V_{-}\right)$containing $p_{-}^{*}$ and $\mathbb{F} \mathbb{P}\left(V_{-}\right)$. As in the spherical section case, this extends 
to a well-defined G-equivariant diffeomorphism from $M$ to $\mathbb{F P}\left(V_{+} \oplus V_{-}\right)$, by invariance, and we are done.

Finally, let us consider the only remaining case (b), where $B_{-}=\mathbb{C P}^{1}$ and $B_{+}$is a complex projective space of real dimension at least 4 , since the cases of $\operatorname{dim} B_{+} \leqslant 2$ reduce to the fixed-point case, because $\operatorname{dim} \Delta^{+} \geqslant 1$.

Since $\mathrm{G}$ acts transitively on $B_{-}=\mathbb{C} \mathbb{P}^{1}=\mathbb{S}^{2}$, we can write $\mathrm{G}=\mathrm{K}_{-} \cdot \mathrm{S}^{3}$, with $\mathrm{K}_{-}$being the kernel of the action on $B_{-}$. Hence, $\mathrm{G}_{-}=\mathrm{K}_{-} \cdot \mathrm{S}^{1}$, where $\mathrm{S}^{1} \subset \mathrm{S}^{3}$. By Lemma 7.2, the subaction of $\mathrm{G}_{-}$is orbit-equivalent to the $\mathrm{G}$-action on $B_{+}$. It follows from Lemma 7.8 below that the factor $\mathrm{S}^{3}$ acts trivially on $B_{+}$, that is $\mathrm{S}^{3} \triangleleft \mathrm{K}_{+}$, the kernel of the $\mathrm{G}$-action on $B_{+}$. Thus, the slice subrepresentation of $\mathrm{K}_{+} \triangleright \mathrm{S}^{3}$ on $V_{+} \cong \mathbb{C}^{2}$ descends to the transitive action on $B_{-}$. This implies that $\mathrm{K}_{+}=\mathrm{S}^{3}$ or $\mathrm{U}(2)$, and accordingly $\mathrm{K}_{0}=\mathrm{K}_{+} \cap \mathrm{K}_{-}$is either 1 or $\mathrm{S}^{1}$. We are now in a situation similar to case (a) with $\mathbb{F}=\mathbb{C}$, and hence $M$ is $\mathrm{G}$ equivariantly diffeomorphic to the complex projective space $\mathbb{P}\left(V_{+} \oplus V_{-}\right)$. This completes the proof.

In conclusion, here are the facts that we used about representations in the proof of Theorem 7.1.

LEMmA 7.7. Let $\varrho: \mathrm{G} \rightarrow \mathrm{SO}\left(V_{0} \oplus \ldots \oplus V_{k}\right)$, be a reducible polar representation, where the $V_{i}$ are irreducible G-modules $(k \geqslant 1)$. Suppose that the $\mathrm{G}$-action on the unit sphere $\mathbb{S}\left(V_{0} \oplus \ldots \oplus V_{k}\right)$ descends to a polar action on the projective space $\mathbb{F} \mathbb{P}\left(V_{0} \oplus \ldots \oplus V_{k}\right)$ where $\mathbb{F}=\mathbb{C}$ or $\mathbb{H}$. If $\operatorname{dim} V_{0} \geqslant 5$ and $\operatorname{dim} V_{0} / \mathrm{G}=1$, then there is a normal simple subgroup $\mathrm{H} \triangleleft \mathrm{G}$ of rank at least 2 acting transitively on the unit sphere $\mathbb{S}\left(V_{0}\right)$ but trivially on $V_{1} \oplus \ldots \oplus V_{k}$.

Proof. Since $\mathrm{G}$ is transitive on $\mathbb{S}\left(V_{0}\right)$, by the list of transitive actions on the spheres it follows that $\mathrm{G}=\mathrm{H} \cdot \mathrm{G}^{\prime}$, where $\mathrm{H}$ is a simple normal subgroup of $\mathrm{G}$ acting transitively on $\mathbb{S}\left(V_{0}\right)$, with principal isotropy group $\mathrm{H}_{0}$. For dimension reason, rank $\mathrm{H} \geqslant 2$. Moreover, $\mathrm{H}$ is a special unitary group or a sympletic group, since it acts transitively on the projective space $\mathbb{F} \mathbb{P}\left(V_{0}\right)$.

We argue by contradiction. Assume that $\mathrm{H}$ acts non-trivially on $V_{i}$ for some $i \geqslant 1$. For the restricted reducible polar representation of $\mathrm{G}$ on $V_{0} \oplus V_{i}$, since the principal isotropy group of $\mathrm{G}$ on $V_{0}$ is $\mathrm{H}_{0} \cdot \mathrm{G}^{\prime}$, by [Be, Theorem 2] it follows that $\mathrm{G}$ is orbit-equivalent to $\mathrm{H}_{0} \cdot \mathrm{G}^{\prime}$ on $V_{i}$, hence, by [Dad], orbit-equivalent to the isotropy representation of an irreducible symmetric space. Note that $\mathrm{H}_{0} \varsubsetneqq \mathrm{H}$ is not a normal subgroup, and by assumption $\mathrm{H}$ is non-trivial on $V_{i}$. By the list in $[\mathrm{EH}]$ it follows that, if $\operatorname{dim} V_{i} / \mathrm{G} \geqslant 2$, then $\mathrm{H}$ is either Spin(8) or Spin(7), which is neither a unitary nor a symplectic group, yielding a contradiction.

It remains to consider the case where $\operatorname{dim} V_{i} / \mathrm{G}=1$, i.e., $\mathrm{G}$ acts transitively on $\mathbb{S}\left(V_{i}\right)$. By the assumption that $\mathrm{H}$ acts non-trivially on $V_{i}$, from the list of transitive actions on 
spheres it follows that

- the simple group $\mathrm{H}$, of rank at least 2, acts transitively on $\mathbb{S}\left(V_{i}\right)$. Furthermore, by dividing the kernel of the $\mathrm{G}^{\prime}$-action, the transitive action of $\mathbf{H} \cdot \mathrm{G}^{\prime}$ on $\mathbb{S}\left(V_{i}\right)$ reduces to an almost effective action of $\mathrm{H} \cdot \mathrm{K}_{0}$ where $\operatorname{rank} \mathrm{K}_{0} \leqslant 1$.

Note that $\operatorname{dim} \mathbb{S}\left(V_{i}\right) \geqslant 4$, since a simple group of rank at least 2 can not act nontrivially on a lower-dimensional sphere. Recall that $\mathrm{H}_{0} \cdot \mathrm{G}^{\prime}$ is orbit-equivalent to the $\mathrm{H} \cdot \mathrm{G}^{\prime}$-action on $V_{i}$, i.e., transitive on $\mathbb{S}\left(V_{i}\right)$. For dimension reasons it follows that the $\mathrm{H}_{0}$ action is also transitive on $\mathbb{S}\left(V_{i}\right)$, since the $\mathrm{G}^{\prime}$-action modulo kernel reduces to the $\mathrm{K}_{0}$-action where $\operatorname{rank} \mathrm{K}_{0} \leqslant 1$.

In summary, $\mathrm{H}$ acts transitively on $\mathbb{S}\left(V_{i}\right)$ orbit-equivalent to the subaction of $\mathrm{H}_{0} \varsubsetneqq \mathrm{H}$ on $\mathbb{S}\left(V_{i}\right)$ where $\mathbf{H} / \mathrm{H}_{0} \cong \mathbb{S}\left(V_{0}\right)$. Recall that $\mathrm{H}$ is a special unitary group or a symplectic group. From the list of transitive actions on spheres, it follows that $\mathrm{H}=\mathrm{SU}(4)$ acts on $V_{0} \oplus V_{i}=\mathbb{R}^{8} \oplus \mathbb{R}^{6}$ via the standard complex representation and the real representation of $\mathrm{SU}(4)=\operatorname{Spin}(6)$ on the summands (cf. [Be]). Since the Spin(6) action on $\mathbb{R}^{6}$ does not descend to the complex projective plane, a contradiction is reached.

In the proof of the following result, we will freely use the language and results about chamber systems and their universal covers developed in $\S 4$, translated to the current setting.

Lemma 7.8. Let $\varrho: \mathrm{G} \rightarrow \mathrm{SO}(V)$ be an almost effective $\mathbb{C}$-linear polar representation, descending to a polar action on the projective space $\mathbb{C P}(V)$.

Suppose $\mathrm{G}=\mathrm{K} \cdot \mathrm{S}^{1} \subset \mathrm{G}^{\prime}=\mathrm{K} \cdot \mathrm{S}^{3}$, and assume moreover that $\mathrm{G}^{\prime}$ acts on the projective space extending the $\mathrm{G}$-action with the same orbits. Then the factor $\mathrm{S}^{3}$ of $\mathrm{G}^{\prime}$ is in the kernel of the action on $\mathbb{C P}(V)$.

Proof. We first prove that the factor $\mathrm{S}^{3}$ action is trivial on $\mathbb{C P}\left(V_{i}\right)$ for each irreducible summand $V_{i} \subset V$. This is clear for any rank-1 summand (summand $V_{i}$ with $\operatorname{dim} V_{i} / \mathrm{G}=1$ ), since $\mathrm{G}^{\prime} \supset \mathrm{G}$ acts transitively on $\mathbb{C P}\left(V_{i}\right)$, hence modulo kernel it is either a unitary group or a symplectic group. If the rank of a summand is 2 , it follows immediately from [Uch]. If the rank of a summand is at least 3 , by $\S 4$, the action of $\mathrm{G}^{\prime} \supset \mathrm{G}$ lifts to a polar representation by an extension by the deck transformation group $S^{1}$, i.e., $S^{1} \cdot G^{\prime}$, acting on the universal cover, i.e., $\mathbb{S}\left(V_{i}\right)$, orbit-equivalent to the $\mathbb{S}^{1} \cdot \mathrm{G}$-representation. By the classification in $[\mathrm{EH}]$, it follows that the $S^{3}$ factor, as well as the factor $S^{1} \subset S^{3}$, must act trivially on $\mathbb{S}\left(V_{i}\right)$. In general, suppose that $\mathrm{S}^{3}$ acts trivially on both $\mathbb{C P}\left(V_{i}\right)$ and $\mathbb{C P}\left(V_{j}\right)$; then $\mathrm{S}^{3}$ acts on $\mathbb{C P}\left(V_{i} \oplus V_{j}\right)$, with the disjoint union $\mathbb{C P}\left(V_{i}\right) \sqcup \mathbb{C P}\left(V_{j}\right)$ as fixed-point set. As the space of geodesics $\Gamma\left(p_{i}\right) \cap \Gamma\left(p_{j}\right)$ joining $p_{i} \in \mathbb{C P}\left(V_{i}\right)$ and $p_{j} \in \mathbb{C P}\left(V_{j}\right)$ is an $\mathbb{S}^{1}$-family of geodesics, $\mathrm{S}^{3}$ must act trivially on that as well, for all $p_{i}$ and $p_{j}$. Thus, $\mathrm{S}^{3}$ acts trivially on $\mathbb{C P}\left(V_{i} \oplus V_{j}\right)$. By induction, it follows that the $\mathrm{S}^{3}$-action is trivial on $\mathbb{C P}(V)$. 
The following is probably a well-known result.

LEMMA 7.9. Let $\varrho: \mathrm{G} \rightarrow \mathrm{SO}(V)$ be an almost effective representation descending to $\mathbb{H} \mathbb{P}(V)=\mathbb{H} \mathbb{P}^{n}$. Then the kernel of the action on $\mathbb{H P}(V)$ is contained in $\mathrm{Sp}(1)$.

Proof. Identify $V$ with the tangent space at a point $p \in \mathbb{H} \mathbb{P}^{n+1}$. The representation gives rise to an isometric action on $\mathbb{H} \mathbb{P}^{n+1}$ fixing $p$ and with the induced action on $\mathbb{H} \mathbb{P}^{n}$ identified as the cut locus of $p$ in $\mathbb{H} \mathbb{P}^{n+1}$. Since the subgroup of the isometry group of $\mathbb{H} \mathbb{P}^{n+1}$ that fixes $\mathbb{H} \mathbb{P}^{n}$ is $\operatorname{Sp}(1)$, the claim follows.

\section{References}

[A] Alexandrino, M. M., On polar foliations and the fundamental group. Results Math., 60 (2011), 213-223.

[AT] Alexandrino, M. M. \& TöBen, D., Singular Riemannian foliations on simply connected spaces. Differential Geom. Appl., 24 (2006), 383-397.

[Be] Bergmann, I., Reducible polar representations. Manuscripta Math., 104 (2001), 309-324.

[Br] Browder, W., Higher torsion in H-spaces. Trans. Amer. Math. Soc., 108 (1963), 353-375.

[BS] Burns, K. \& Spatzier, R., On topological Tits buildings and their classification. Inst. Hautes Études Sci. Publ. Math., 65 (1987), 5-34.

[CL] Charney, R. \& Lytchak, A., Metric characterizations of spherical and Euclidean buildings. Geom. Topol., 5 (2001), 521-550.

[C] Cheeger, J., Some examples of manifolds of nonnegative curvature. J. Differential Geometry, 8 (1973), 623-628.

[Dad] DAdok, J., Polar coordinates induced by actions of compact Lie groups. Trans. Amer. Math. Soc., 288 (1985), 125-137.

[Dav] Davis, M. W., The Geometry and Topology of Coxeter Groups. London Mathematical Society Monographs Series, 32. Princeton University Press, Princeton, NJ, 2008.

[De] Dearricott, O., A 7-manifold with positive curvature. Duke Math. J., 158 (2011), 307-346.

[EH] Eschenburg, J.-H. \& Heintze, E., On the classification of polar representations. Math. Z., 232 (1999), 391-398.

[FG] FAng, F. \& Grove, K., Reflection groups in non-negative curvature. J. Differential Geom., 102 (2016), 179-205.

[FGT] Fang, F., Grove, K. \& Thorbergsson, G., Rank three geometry and positive curvature. Comm. Anal. Geom., 24 (2016), 487-520.

[FKM] Ferus, D., Karcher, H. \& Münzner, H.F., Cliffordalgebren und neue isoparametrische Hyperflächen. Math. Z., 177 (1981), 479-502.

[GKo] Gorodski, C. \& Kollross, A., Some remarks on polar actions. Ann. Global Anal. Geom., 49 (2016), 43-58.

[Gr] Grove, K., Developments around positive sectional curvature, in Geometry, Analysis, and Algebraic Geometry, Surv. Differ. Geom., 13, pp. 117-133. Int. Press, Somerville, MA, 2009. 
[GKi] Grove, K. \& Kim, C. W., Positively curved manifolds with low fixed point cohomogeneity. J. Differential Geom., 67 (2004), 1-33.

[GVZ] Grove, K., Verdiani, L. \& Ziller, W., An exotic $T_{1} \mathbb{S}^{4}$ with positive curvature. Geom. Funct. Anal., 21 (2011), 499-524.

[GWZ] Grove, K., Wilking, B. \& Ziller, W., Positively curved cohomogeneity one manifolds and 3-Sasakian geometry. J. Differential Geom., 78 (2008), 33-111.

[GZ] Grove, K. \& Ziller, W., Polar manifolds and actions. J. Fixed Point Theory Appl., 11 (2012), 279-313.

[GKMW] Grundhöfer, T., Kramer, L., Van Maldeghem, H. \& Weiss, R. M., Compact totally disconnected Moufang buildings. Tohoku Math. J., 64 (2012), 333-360.

[Ha] Hatcher, A., Algebraic Topology. Cambridge University Press, Cambridge, 2002.

[HPtT] Heintze, E., Palais, R.S., Terng, C. L. \& Thorbergsson, G., Hyperpolar actions on symmetric spaces, in Geometry, Topology, 83 Physics, Conf. Proc. Lecture Notes Geom. Topology, 4, pp. 214-245. Int. Press, Cambridge, MA, 1995.

[He] Helgason, S., Differential Geometry, Lie Groups, and Symmetric Spaces. Graduate Studies in Mathematics, 34. Amer. Math. Soc., Providence, RI, 2001.

[I] Iwata, K., Classification of compact transformation groups on cohomology quaternion projective spaces with codimension one orbits. Osaka J. Math., 15 (1978), $475-508$.

[KL] Kramer, L. \& Lytchak, A., Homogeneous compact geometries. Transform. Groups, 19 (2014), 793-852.

[L] Lytchak, A., Polar foliations of symmetric spaces. Geom. Funct. Anal., 24 (2014), 1298-1315.

[M] Mendes, R. A. E., Extending tensors on polar manifolds. Math. Ann., 365 (2016), 1409-1424.

[MZ] Montgomery, D. \& Zippin, L., Topological Transformation Groups. Interscience Publishers, New York-London, 1955.

[N] Neumaier, A., Some sporadic geometries related to PG(3,2). Arch. Math., 42 (1984), 89-96.

[O] Onishchik, A. L., Topology of Transitive Transformation Groups. Johann Ambrosius Barth, Leipzig, 1994.

[OT] OzeKI, H. \& TAKEUCHI, M., On some types of isoparametric hypersurfaces in spheres. II. Tôhoku Math. J., 28 (1976), 7-55.

[PTe] Palais, R.S. \& Terng, C.-L., A general theory of canonical forms. Trans. Amer. Math. Soc., 300 (1987), 771-789.

[Pe] Petersen, P., Riemannian Geometry. Graduate Texts in Mathematics, 171. Springer, New York, 2006.

[PTh] Podestà, F. \& Thorbergsson, G., Polar actions on rank-one symmetric spaces. J. Differential Geom., 53 (1999), 131-175.

[Po] Poncet, J., Groupes de Lie compacts de transformations de l'espace euclidien et les sphères comme espaces homogènes. Comment. Math. Helv., 33 (1959), 109-120.

[R] Ronan, M., Lectures on Buildings. Perspectives in Mathematics, 7. Academic Press, Boston, MA, 1989.

[Sz] Szenthe, J., Orthogonally transversal submanifolds and the generalizations of the Weyl group. Period. Math. Hungar., 15 (1984), 281-299.

[Th] Thorbergsson, G., Isoparametric foliations and their buildings. Ann. of Math., 133 (1991), 429-446.

[Ti1] Tits, J., Buildings of Spherical Type and Finite BN-Pairs. Lecture Notes in Mathematics, 386. Springer, Berlin-New York, 1974. 
[Ti2] - A local approach to buildings, in The Geometric Vein, pp. 519-547. Springer, New York-Berlin, 1981.

[Ucc] UCCI, J., On the nonexistence of Riemannian submersions from $\mathbf{C P}(7)$ and $\mathrm{QP}(3)$. Proc. Amer. Math. Soc., 88 (1983), 698-700.

[Uch] UCHIDA, F., Classification of compact transformation groups on cohomology complex projective spaces with codimension one orbits. Japan. J. Math., 3 (1977), 141189.

[V] Verdiani, L., Cohomogeneity one manifolds of even dimension with strictly positive sectional curvature. J. Differential Geom., 68 (2004), 31-72.

[VZ] Verdiani, L. \& Ziller, W., Concavity and rigidity in non-negative curvature. J. Differential Geom., 97 (2014), 349-375.

[Wa] Walschap, G., Metric foliations and curvature. J. Geom. Anal., 2 (1992), 373-381.

[Wi1] Wilking, B., Positively curved manifolds with symmetry. Ann. of Math., 163 (2006), 607-668.

[Wi2] - A duality theorem for Riemannian foliations in nonnegative sectional curvature. Geom. Funct. Anal., 17 (2007), 1297-1320.

[Wi3] - Nonnegatively and positively curved manifolds, in Surveys in Differential Geometry, Vol. XI, pp. 25-62. Int. Press, Somerville, MA, 2007.

[Wö] Wörner, A., A splitting theorem for nonnegatively curved Alexandrov spaces. Geom. Topol., 16 (2012), 2391-2426.

[Z] Ziller, W., Examples of Riemannian manifolds with non-negative sectional curvature, in Surveys in Differential Geometry, Vol. XI, pp. 63-102. Int. Press, Somerville, MA, 2007.

FUQUAN FANG

Department of Mathematics

Capital Normal University

Beijing 100048

China

fuquan_fang@yahoo.com

Gudlaugur Thorbergsson

Mathematisches Institut

Universität zu Köln

Weyertal 86-90

50931 Köln

Germany

gthorber@mi.uni-koeln.de

Received March 30, 2015

Received in revised form April 17, 2016

\section{Karsten Grove}

Department of Mathematics

University of Notre Dame

Notre Dame, IN 46556

U.S.A.

kgrove2@nd.edu 